GUSTAVO TORRENTS SCHMIDT

\title{
MODELAGEM DA COALESCÊNCIA EM SISTEMAS BIFÁSICOS POLIDISPERSOS USANDO BALANÇO POPULACIONAL E TÉCNICAS DE CFD - APLICAÇÃO À DESSALGAÇÃO DE PETRÓLEO
}

Dissertação apresentada à Escola Politécnica da Universidade de São Paulo para a obtenção do título de Mestre em Engenharia

São Paulo 
GUSTAVO TORRENTS SCHMIDT

\section{MODELAGEM DA COALESCÊNCIA EM SISTEMAS BIFÁSICOS POLIDISPERSOS USANDO BALANÇO POPULACIONAL E TÉCNICAS DE CFD - APLICAÇÃO À DESSALGAÇÃO DE PETRÓLEO}

Dissertação apresentada à Escola Politécnica da Universidade de São Paulo para a obtenção do título de Mestre em Engenharia

Área de Concentração:

Engenharia Química

Orientador:

Roberto Guardani

São Paulo 


\section{RESUMO}

O balanço populacional é um método comprovado de se aumentar a previsibilidade do comportamento de um sistema multifásico, e sua utilização em conjunto de técnicas de CFD tem sido cada vez maior pelo desenvolvimento constante de ambas as tecnologias.

Este trabalho apresenta o equacionamento genérico do balanço populacional para sistemas bifásicos com agregação e quebra de partículas, além de uma discussão sobre a natureza de sistemas bifásicos. Métodos numéricos específicos para a resolução deste tipo de problema são discutidos, implementados e validados.

Como exemplo de aplicação do equacionamento sugerido, é obtido um modelo específico para o caso de coalescência de gotas de água salgada dispersas numa fase óleo submetidas a um campo elétrico alternado, como no processo de dessalgação de petróleo. Um algoritmo baseado em autômatos celulares é utilizado como fonte de dados para validação do modelo e técnicas de CFD produzem um perfil de escoamento da emulsão.

Palavras-chave: Dessalinização; Petróleo; Fenômenos de Transporte. 


\begin{abstract}
The population balance is a proven method for increasing a multiphase system's behavior predictability, and its employment along with CFD techniques is increasing following the constant development of both technologies.

This work presents the generic Population Balance Equations for two-phase systems where its particles suffer aggregation and breakage and a discussion on the nature of two-phase systems. Specific numerical methods for the solution of such problems are discussed, implemented and validated.

A specific model for the coalescence of water droplets dispersed in an oily phase under the effects of an alternated electric field is obtained as an application example of the suggested equations, mimicking the oil desalting process. A cellular automata based algorithm is used as data source for the model's validation and CFD techniques are used to produce the emulsion's flow profile.
\end{abstract}

Keywords: Desalting; Oil; Transport Phenomena. 


\section{LISTA DE ILUSTRAÇÕES}

Figura 1.3.1 - Esquema da seção transversal de um módulo de dessalgação. 15

Figura 2.1.1 - Notação da discretização por volumes finitos. 17

Figura 2.4.2.1 - Representação gráfica da aproximação por quadratura....... 34

Figura 3.1.1 - Solução do DQMOM para a EBP de McCoy e Madras (2003) com agregação dominante $(\Phi(\infty)=0,1)$

Figura 3.1.2 - Solução do DQMOM para a EBP de McCoy e Madras (2003) com quebra dominante $(\Phi(\infty)=10)$.

Figura 3.1.3 - Solução do DQMOM para a EBP de McCoy e Madras (2003) com distribuição constante $(\Phi(\infty)=1)$.

Figura 3.1.4 - Erro do DQMOM em relação à solução analítica da EBP de McCoy e Madras (2003) com agregação dominante $(\Phi(\infty)=0,1)$

Figura 3.1.5 - Erro do DQMOM em relação à solução analítica da EBP de McCoy e Madras (2003) com quebra dominante $(\Phi(\infty)=10)$.

Figura 3.1.6 - Erro do DQMOM em relação à solução analítica da EBP de McCoy e Madras (2003) com distribuição constante $(\Phi(\infty)=1)$.

Figura 3.2.1 - Erro do DQMOM em relação à solução analítica da EBP de McCoy e Madras (2003) com agregação dominante $(\Phi(\infty)=0,1)$ e $N=2$ pontos de quadratura.

Figura 3.2.2 - Erro do DQMOM em relação à solução analítica da EBP de McCoy e Madras (2003) com agregação dominante $(\Phi(\infty)=0,1)$ e $N=3$ pontos de quadratura.

Figura 3.2.3 - Erro do DQMOM em relação à solução analítica da EBP de McCoy e Madras (2003) com agregação dominante $(\Phi(\infty)=0,1)$ e $N=4$ pontos de quadratura.

Figura 3.2.4 - Erro do DQMOM em relação à solução analítica da EBP de McCoy e Madras (2003) com agregação dominante 50 
$(\Phi(\infty)=0,1)$ e $N=5$ pontos de quadratura

Figura 4.1.1 - Região do módulo de dessalgação considerada por Bresciani (2009), angularmente simétrica em torno do eixo do dispersor.

Figura 4.1.2 - Comportamento dos momentos obtidos pelo algoritmo de Bresciani (2009) para células de $400 \mathrm{~mm} \times 400 \mathrm{~mm}$, $T=120^{\circ} \mathrm{C}, 7,0 \%$ de água, $E=1,0 \mathrm{kV} / \mathrm{cm}$, emulsão média e grandezas adimensionalizadas.

Figura 4.1.3 - Solução do QMOM proposto para um sistema sem escoamento ou difusão com agregação pura e Kernel Constante com $a=19$.

Figura 4.1.4 - Solução do QMOM proposto para um sistema sem escoamento ou difusão com agregação pura e Kernel Soma com $a=0,04$

Figura 4.1.5 - Solução do QMOM proposto para um sistema sem escoamento ou difusão com agregação pura e Kernel Cisalhamento de Smoluchowsky com $a=0,013$.

Figura 4.1.6 - Solução do QMOM proposto para um sistema sem escoamento ou difusão com agregação pura e Kernel Produto com $a=4,6.10^{-4}$ e $b=1$

Figura 4.1.7 - Solução do QMOM proposto para um sistema sem escoamento ou difusão com agregação pura e Kernel de Kapur com $a=4300$ e $b=c=1$

Figura 4.1.8 - Solução do QMOM proposto para um sistema sem escoamento ou difusão com agregação pura e Kernel de Kapur com $a=3,1, b=2$ e $c=1$.

Figura 4.1.9 - Solução do QMOM proposto para um sistema sem escoamento ou difusão com agregação pura e Kernel de Kapur com $a=2,4.10^{9}, b=1$ e $c=2$

Figura 4.1.10 - Solução do QMOM proposto para um sistema sem escoamento ou difusão com agregação pura e Kernel de Sastry com $a=10^{-3}$ 
Figura 4.1.11 - Solução do QMOM proposto para um sistema sem escoamento ou difusão com agregação pura e Kernel de Adetayo e Ennis com $a=0,24, b=1$ e $c=1$.

Figura 4.1.12 - Solução do QMOM proposto para um sistema sem escoamento ou difusão com agregação pura e Kernel de Adetayo e Ennis com $a=9,9 b=1$ e $c=2$.

Figura 4.2.1 - Perfil de escoamento proposto por Bresciani (2009).

Figura 4.2.2 - Geometria do módulo de dessalgação apresentada por Bresciani (2009)

Figura 4.2.3 - Geometria da simulação de escoamento em CFD (A)........ 66

Figura 4.2.4 - Geometria da simulação de escoamento em CFD (B)........ 66

Figura 4.2.5 - Campo de velocidades calculado para a geometria (A)....... 67

Figura 4.2.6 - Campo de velocidades calculado para a geometria (B)...... 67

Figura 4.2.7 - Detalhe do campo de velocidades calculado para a geometria (B)............................................................. 68

Figura 4.2.8 - Tempo de residência do fluido escoando da válvula distribuidora até a reta $\alpha$ para a geometria $(\mathrm{A}) \ldots \ldots \ldots \ldots \ldots \ldots . . . . . . . .68$

Figura 4.2.9 - Tempo de residência do fluido escoando da válvula distribuidora até a reta $\alpha$ para a geometria (B).................. 69

Figura 4.2.10 - Tempo de residência do fluido escoando da válvula distribuidora até a reta $\alpha$ para a geometria (B). Valores extrapolados por todos os $0,2 \mathrm{~m}$ do segmento de reta..........

Figura 4.2.11 - Solução do modelo de agregação proposto, com indicação do tempo de residência mínimo requerido para utilização do modelo $(t=0,4 \mathrm{~s})$ e do obtido com a simulação das

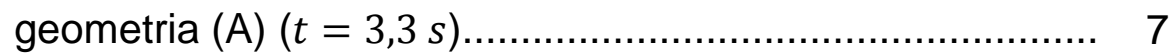




\section{LISTA DE TABELAS}

Tabela 3.1.1 - Pesos e abscissas iniciais para validação do DQMOM........................................................................

Tabela 4.1.1 - Momentos iniciais e finais obtidos pelo algoritmo de Bresciani (2009) para células de 400x400, $T=120^{\circ} \mathrm{C}, 7,0 \%$ de água, $E=1,0 \mathrm{kV} / \mathrm{cm}$, emulsão média e grandezas adimensionalizadas

Tabela 4.1.2 - Tabela 4.1.2 - Funções frequência de agregação testadas.. 56

Tabela 4.1.3 - Momentos finais obtidos pelo modelo proposto e seu erro relativo ao algoritmo de Bresciani (2009) para células de $400 \times 400, T=120^{\circ} \mathrm{C}, 7,0 \%$ de água, $E=1,0 \mathrm{kV} / \mathrm{cm}$, emulsão média e grandezas adimensionalizadas. 


\section{LISTA DE SÍMBOLOS}

a coeficiente; frequência de agregação de pares de partículas

$B \quad$ termo fonte de nascimento de partículas

$\bar{B} \quad$ termo fonte de nascimento de partículas médio

$b \quad$ coeficiente; frequência de quebra de partículas

c coeficiente

D derivada lagrangeana

D termo fonte de desaparecimento de partículas

$\bar{D} \quad$ termo fonte de desaparecimento de partículas médio

d derivada

E campo elétrico

$f \quad$ fator; função densidade numérica média de partículas

$G \quad$ termo fonte de crescimento de partículas

$g$ termo de crescimento independente da coordenada interna; campo gravitacional

j termo difusivo

$k \quad$ constante de valor igual à ordem do momento $m_{k}$

L $\quad$ comprimento característico

$M \quad$ termo de transferência de quantidade de movimento

$m$ massa; momento (com subscrito)

max máximo

min mínimo

$N$ número total esperado de partículas; termo fonte de nucleação; número de pontos de quadratura

$n$ termo de nucleação independente da coordenada interna; quantidade de gotas

P densidade de probabilidade de origem de partículas por quebra

$\bar{P} \quad$ densidade de probabilidade de origem de partículas por quebra média

$P_{e} \quad$ número de Peclet

$\dot{R} \quad$ velocidade na coordenada espacial

$r \quad$ coordenada espacial

$S \quad$ termo fonte 


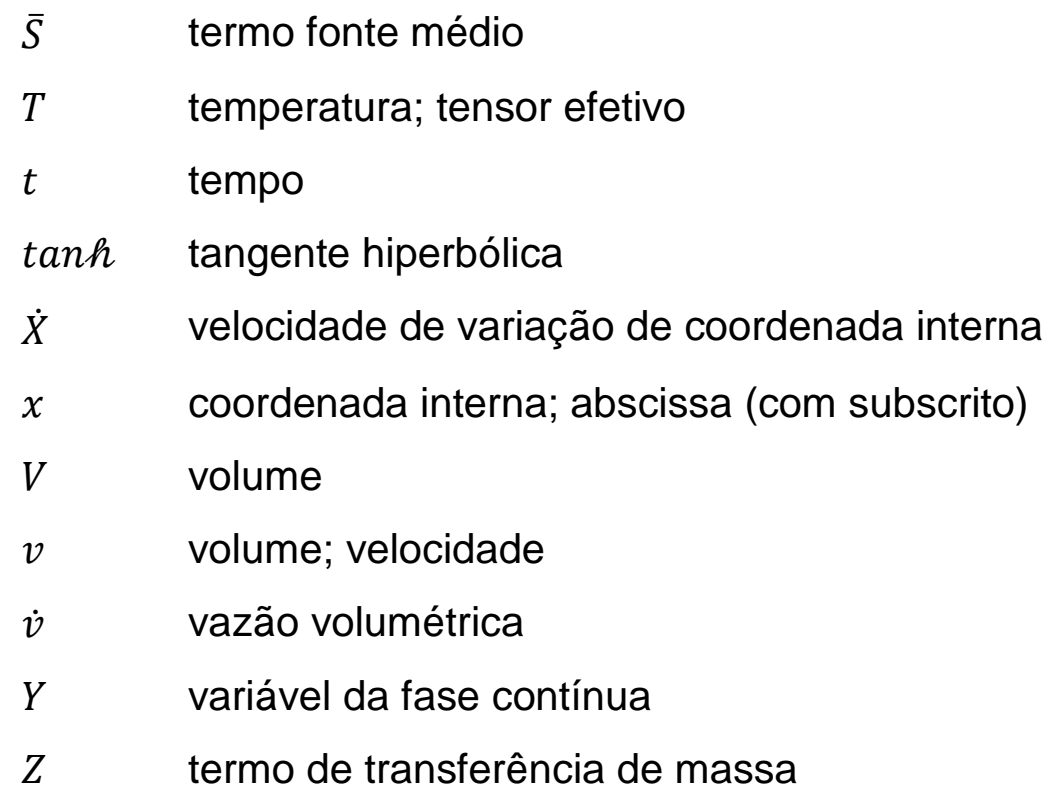

\section{Letras gregas}

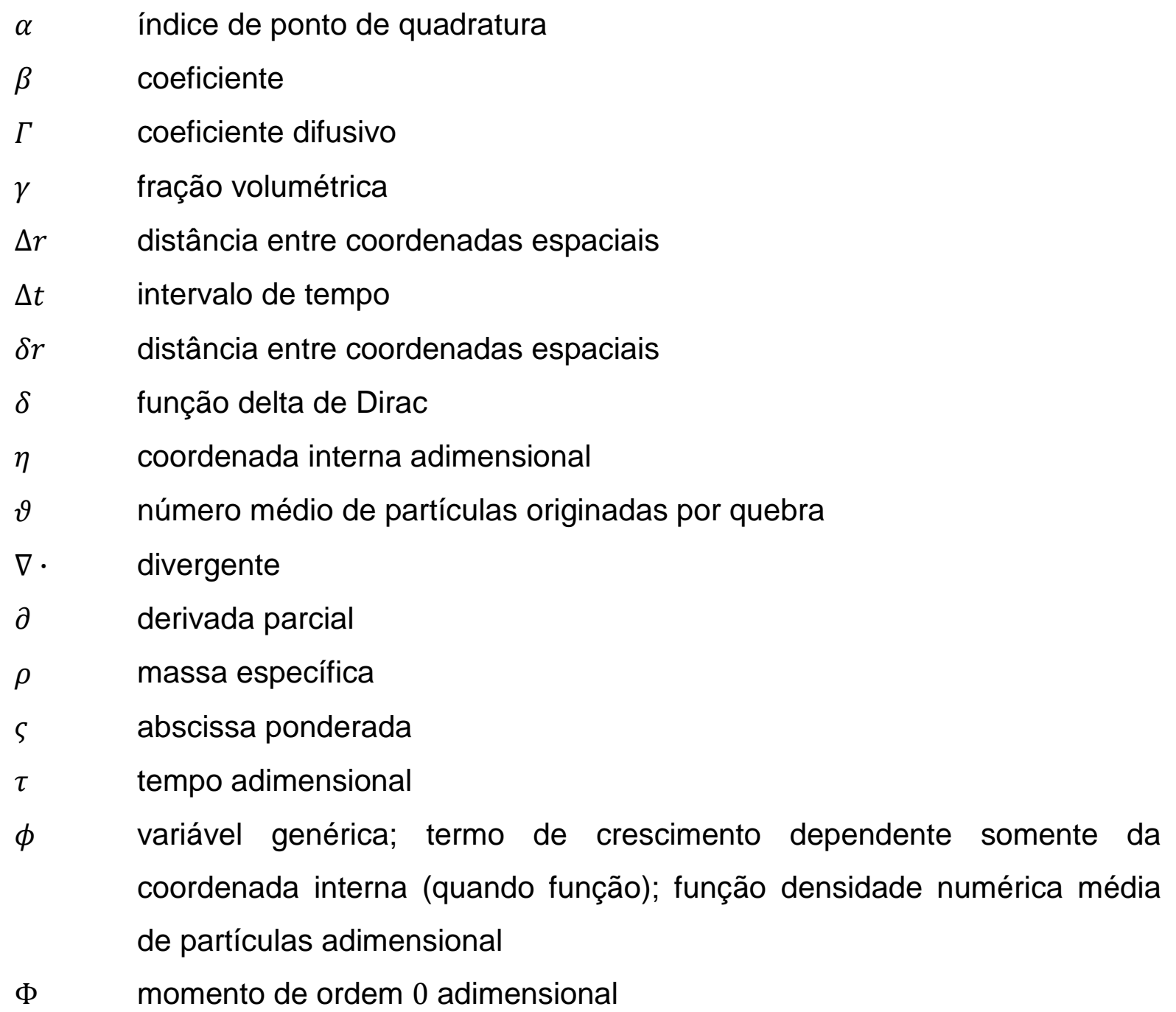


$\psi \quad$ termo de nucleação dependente somente da coordenada interna

$\Omega \quad$ domínio

$\omega \quad$ peso

\section{Subscritos}

$\begin{array}{ll}e & \text { Leste } \\ w & \text { Oeste } \\ p & \text { ponto } \\ c & \text { constante } \\ r & \text { coordenadas espaciais; (tempo) de residência } \\ x & \text { coordenadas internas } \\ d & \text { fase dispersa } \\ k & \text { ordem de momento } \\ \alpha & \text { índice de ponto de quadratura } \\ \omega & \text { relativo a um peso } \\ \varsigma & \text { relativo a uma abscissa ponderada } \\ \beta & \text { índice de ponto de quadratura; índice de fase }\end{array}$

\section{Sobrescritos}
$t \quad$ instante
$t+\Delta t \quad$ instante acrescido de um intervalo de tempo
$0 \quad$ instante inicial
$A \quad$ agregação
$B \quad$ quebra
(N) condicionado ao número de pontos de quadratura
adm adimensional

\section{Realçadores}

Itálico escalar

Negrito vetor 


\section{SUMÁRIO}

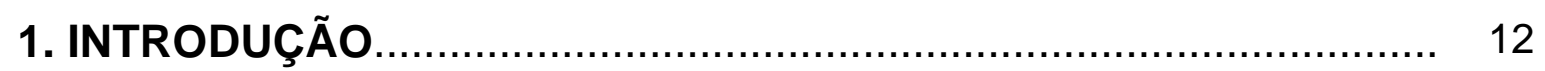

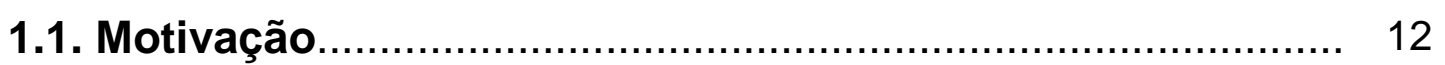

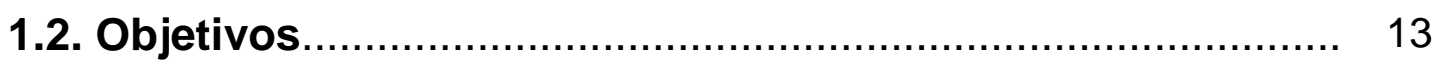

1.3. O Processo de Dessalgação....................................... 14

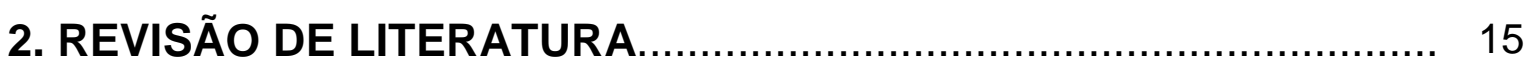

2.1. Fluidodinâmica Computacional (CFD) ......................... 15

2.2. Escoamento Bifásico............................................... 21

2.3. Equação do Balanço Populacional (EBP)....................... 24

2.3.1 Agregação..................................................... 26

2.3.2. Quebra.......................................................... 28

2.4. Métodos Numéricos para a Resolução de Balanços

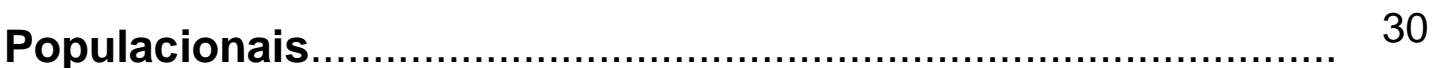

2.4.1. Método dos Momentos (MOM).......................... 31

2.4.2. Método das Quadraturas dos Momentos (QMOM).... 32

2.4.3. Método Direto das Quadraturas dos Momentos

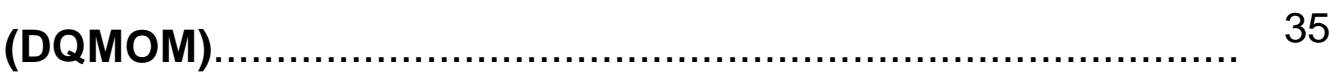

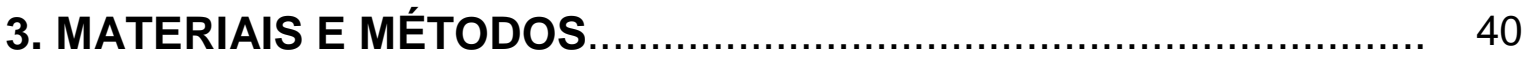

3.1. Reprodução do DQMOM......................................... 40

3.2. Influência do Número de Pontos de Quadratura na

Precisão do DQMOM...................................................... 47

4. RESULTADOS E DISCUSSÃO ........................................... 51

4.1. Aplicação do Balanço Populacional................................ 51

4.2. Modelagem do Escoamento da Fase Contínua................. 64

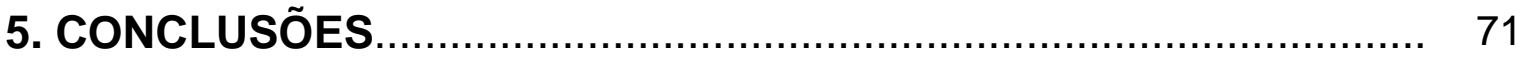

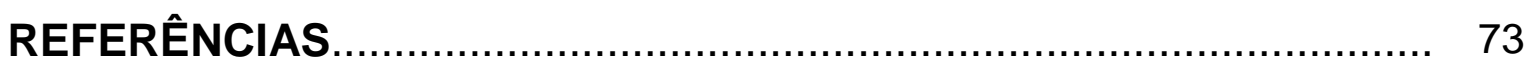

APÊNDICE A - DEMONSTRAÇÃO DA CONSERVAÇÃO DO

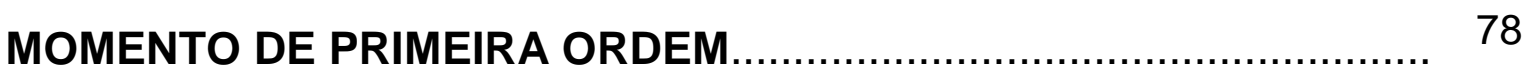




\section{INTRODUÇÃO}

\subsection{Motivação}

Escoamentos bifásicos são caracterizados pela existência de duas fases que formam interfaces. Estes podem ser separados, quando há somente uma interface no sistema, e dispersos, como, por exemplo, partículas de uma fase sólida espalhadas aleatoriamente numa fase líquida, a qual é dita contínua. Quando essas partículas, gotas ou bolhas da fase dispersa possuem diferenças entre si, como temperatura ou tamanho, diz-se que o sistema é polidisperso.

Sistemas polidispersos são comuns em engenharia. Pode-se citar ciclones, decantadoras e colunas de borbulhamento como exemplos comuns de equipamentos onde haja presença de duas ou mais fases nessa condição. Para o último, ainda, a interface média pode variar ao longo da coluna por agregação ou quebra de bolhas que, tendo alguma propriedade que as diferencia como tamanho ou densidade, deslocam-se a velocidades distintas e se chocam. Essas interações afetam a eficiência do processo ao aumentar ou diminuir a capacidade de transferência de quantidade de movimento, calor ou massa entre as fases, podendo também alterar o regime de escoamento dependendo da intensidade dessas interações. Pode-se ver que a capacidade de prever e manipular essas interações é imprescindível nesse caso.

Há basicamente duas formas de equacionar esse tipo de sistema. A conceitualmente mais simples consiste em considerar cada bolha do sistema como uma fase distinta, assim como a fase contínua. A quantidade de equações de transporte necessárias, somadas ainda com a quantificação da evolução das interfaces torna essa abordagem, na maioria dos casos, intratável. Assim, é comumente adotado um equacionamento médio temporal do sistema, que considera ambas as fases coexistindo no mesmo espaço e modela uma interface média entre ambas por meio do balanço populacional.

Mesmo esse tipo de tratamento simplificado requer um esforço computacional significativo. Dessa forma, uma grande quantidade de métodos numéricos 
específicos para esse problema vem sido propostos desde a concepção do Método dos Momentos em 1964. Esse trabalho pioneiro propôs o acompanhamento dos momentos da distribuição de partículas do sistema, priorizando eficiência computacional em detrimento de detalhes da população considerados desnecessários para a maior parte das aplicações de balanço populacional. Essa abordagem continua sendo explorada e expandida atualmente, com métodos como o Método Direto das Quadraturas dos Momentos (FAN; MARCHISIO; FOX, 2004) e o Método Direto das Quadraturas dos Momentos Adaptativo (SU et al., 2008), entre outros.

A popularização de técnicas de Fluidodinâmica Computacional (CFD) também tem contribuído para o desenvolvimento de estudos de sistemas polidispersos. Pelo balanço populacional ser, como o nome diz, um balanço, a discretização espacial e temporal do CFD pode ser aplicada diretamente, assim como seus métodos numéricos acessórios, o que faz com que o acoplamento dos transportes de quantidade de movimento, massa e calor com o de quantidade de partículas em um sistema seja natural.

Finalmente, a possibilidade de ganhos significativos na eficiência de diversos processos usuais da indústria, aliada ao constante desenvolvimento de novas técnicas tanto de CFD quanto de resolução e formulação de balanços populacionais encorajam uma revisão dessas tecnologias e sua aplicabilidade para possibilitar mais avanços nessa área.

\subsection{Objetivos}

Busca-se apresentar o equacionamento genérico de dispersões líquidolíquido nas quais haja clara distinção entre uma fase contínua e uma dispersa, sendo a variação da interface entre as duas regida somente pela agregação ou coalescência das gotas existentes no sistema. É também objeto do trabalho mostrar um exemplo de aplicação do equacionamento proposto, especificamente para 0 processo de dessalgação de petróleo realizado comumente em refinarias e para cuja operação eficiente a manipulação da distribuição de tamanho de gotas da fase dispersa é fundamental. 


\subsection{O Processo de Dessalgação}

O petróleo extraído dos campos de produção sempre contém algum teor de água e sais, seja devido às condições naturais do depósito ou às técnicas utilizadas para sua exploração. O processo de remoção do sal submete a mistura a escoamentos turbulentos que geram uma emulsão de água em óleo estabilizada pelos tensoativos componentes do petróleo. Apesar de grande parte da água ser removida em campo, o petróleo destinado às refinarias possui uma concentração ainda elevada de sais e água emulsionada que precisam ser removidos para prevenir avarias aos equipamentos que possibilitam o processo de refino. Para tanto, existem várias técnicas e equipamentos distintos, baseadas na desestabilização da emulsão por meios químicos ou físicos.

O processo de dessalgação estudado neste trabalho é aquele no qual se baseou o estudo de Bresciani (2009), da dessalgadora eletrostática de alta velocidade. Este pode ser dividido em duas etapas consecutivas: primeiramente, a emulsão, à qual foi adicionada uma corrente de água, é submetida a um escoamento altamente turbulento, diminuindo o tamanho das gotas de água suspensas e aumentando a sua área interfacial, o que promove o transporte dos sais diluídos na fase óleo para a fase água pela possibilidade de uma maior estabilidade termodinâmica. Na sequência a mistura é injetada em um tanque cilíndrico em um dos seus vários módulos de dessalgação de seção circular, onde é submetida a uma corrente elétrica alternada que alinha as gotas de água salgada e as faz vibrar, promovendo sua agregação. Ao alcançar um certo tamanho as gotas de água coalescem, possibilitando a remoção do petróleo virtualmente livre de sais pela parte superior da dessalgadora e da água, rica em sais e contaminantes orgânicos, pela inferior. A figura 1.3.1 ilustra um módulo de dessalgação de alta velocidade: 


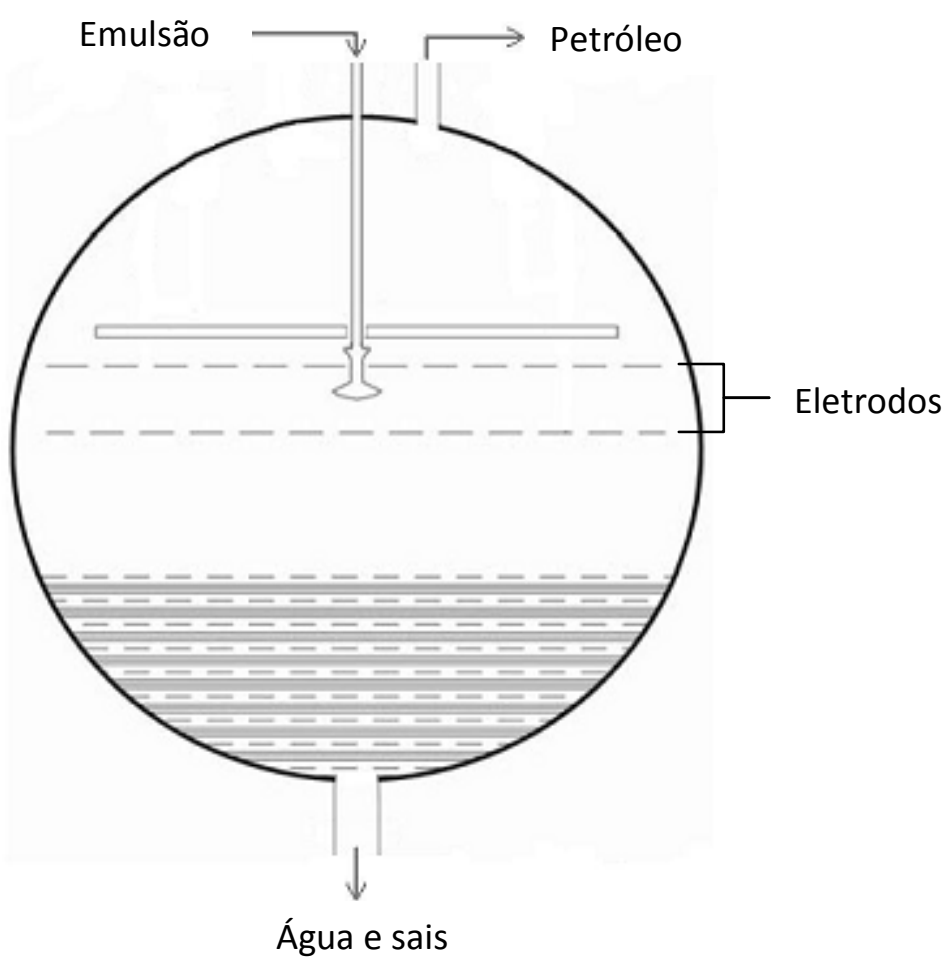

Figura 1.3.1 - Esquema da seção transversal de um módulo de dessalgação

\section{REVISÃO DE LITERATURA}

\subsection{Fluidodinâmica Computacional (CFD)}

Tendo como base uma equação de balanço na forma generalizada (BIRD; STEWART; LIGHTFOOT, 2002), para uma variável extensiva genérica $\phi$ :

$$
\frac{\mathrm{D}[\rho \phi]}{\mathrm{D} t}=\frac{\partial[\rho \phi]}{\partial t}+\nabla \cdot[\rho \dot{\boldsymbol{R}}(\phi, \boldsymbol{r}, t) \phi]=-\nabla \cdot \boldsymbol{j}(\phi, \boldsymbol{r}, t)+S(\phi, \boldsymbol{r}, t)
$$

É possível obter uma forma discretizada da mesma de diferentes maneiras, tais como aproximação por séries de Taylor ou o método dos resíduos ponderados (PATANKAR, 1980), mas o método mais comumente adotado para escoamentos internos é o chamado método dos volumes finitos (VERSTEEG; MALALASEKERA,1995). A principal característica deste é preservar os balanços de 
propriedades no volume de controle, por ter como base a forma integrada da equação diferencial apresentada. Será apresentada somente a formulação correspondente ao mesmo. Uma discussão comparativa entre este e os outros métodos populares no campo da engenharia, o das diferenças finitas e 0 dos elementos finitos, foi realizada por Maliska (1995).

\section{Discretização por Volumes Finitos}

A forma mais simples de discretizar a equação de balanço geral é fazê-lo para o caso estacionário unidirecional e estender o resultado obtido para os casos mais gerais. Para descrever essas condições, de um ponto de vista euleriano, tem-se, de 2.1.1:

$$
\frac{\mathrm{d}[\rho \dot{R}(\phi, r, t) \phi]}{\mathrm{d} r}=-\frac{\mathrm{d} j(\phi, r, t)}{\mathrm{d} r}+S(\phi, r, t)
$$

Admitindo-se que o termo difusivo segue uma lei equivalente à lei de Fourier para o transporte de calor num meio:

$$
j(\phi, r, t)=-\Gamma \frac{\mathrm{d} \phi}{\mathrm{d} r}
$$

A forma integrada do balanço pode ser escrita como:

$$
[\rho \dot{R}(\phi, r, t) \phi]_{e}-[\rho \dot{R}(\phi, r, t) \phi]_{w}=\left[\Gamma \frac{\mathrm{d} \phi}{\mathrm{d} r}\right]_{e}-\left[\Gamma \frac{\mathrm{d} \phi}{\mathrm{d} r}\right]_{w}+\int_{w}^{e} S(\phi, r, t) \mathrm{d} r
$$

Que é a equação a ser discretizada. Os subscritos $w$ e e correspondem às faces diretamente a Oeste e a Leste do elemento que contém o seu ponto de referência, $P$, numa malha estruturada (figura 2.1.1).

A equação mostra que os termos fonte, difusivo e convectivo podem ser tratados independentemente sem afetar os demais, o que possibilita a utilização de aproximações distintas para cada um, úteis dependendo do conhecimento prévio do fenômeno estudado. 
O primeiro passo da discretização é escolher uma interpolação adequada para representar a evolução de $\phi$ na malha. O termo difusivo, por exemplo, pode ser representado por um perfil linear por partes, resultando em:

$$
\left[\Gamma \frac{\mathrm{d} \phi}{\mathrm{d} r}\right]_{e}-\left[\Gamma \frac{\mathrm{d} \phi}{\mathrm{d} r}\right]_{w}=\Gamma_{e} \frac{\left[\phi_{E}-\phi_{P}\right]}{[\delta r]_{e}}-\Gamma_{w} \frac{\left[\phi_{P}-\phi_{W}\right]}{[\delta r]_{w}}
$$

Sendo $\phi_{P}, \phi_{W}$ e $\phi_{E}$ os valores de $\phi$ nos pontos $P$ e em seus vizinhos a Oeste $(W)$ e a Leste $(E)$, respectivamente. $[\delta r]_{w}$ é a distância entre os pontos $W$ e $P$ e $[\delta r]_{e}$ a entre os pontos $P$ e $E$, como mostra a figura 2.1.1.

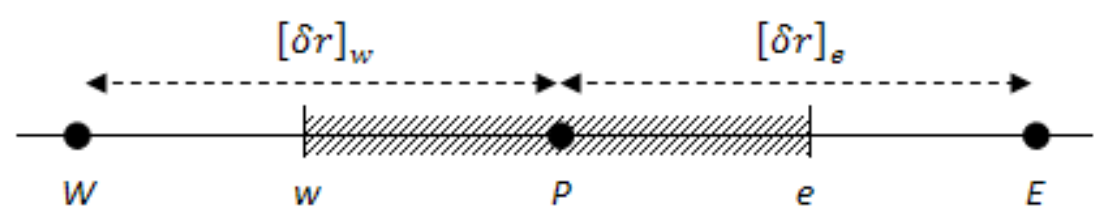

Figura 2.1.1 - Notação da discretização por volumes finitos

O termo fonte pode ser tratado da mesma forma, mas na literatura de CFD adota-se, historicamente, um perfil em degrau para sua representação (PATANKAR, 1980; VERSTEEG; MALALASEKERA, 1995):

$$
\int_{w}^{e} S(\phi, r, t) \mathrm{d} r=\bar{S} \Delta r
$$

Com a aproximação linear

$$
\bar{S}=S_{C}+S_{P} \phi_{P}
$$

Sendo $S_{C}$ a parcela constante do termo fonte e $S_{P}$ o coeficiente de $\phi_{P}$ (e não o valor de $S$ calculado no ponto $P$ ). Essa aproximação admite que o valor do termo fonte é constante no elemento de volume e linearmente dependente de $\phi_{P}$.

Finalmente, a parcela convectiva, se representada por um perfil linear por partes, resulta em: 


$$
\begin{aligned}
{[\rho \dot{R}(\phi, r, t) \phi]_{e} } & -[\rho \dot{R}(\phi, r, t) \phi]_{w} \\
= & {[\rho \dot{R}(\phi, r, t)]_{e} \frac{\left[\phi_{E}+\phi_{P}\right]}{2}-[\rho \dot{R}(\phi, r, t)]_{w} \frac{\left[\phi_{P}+\phi_{W}\right]}{2} }
\end{aligned}
$$

Sendo que o termo $\frac{1}{2}$ aparece ao se admitir interfaces à mesma distância de ambos os seus pontos vizinhos.

Essa forma de representar o termo convectivo é chamada Esquema de Diferenças Centradas. Há outras formas populares, como os esquemas Upwind, Exponencial e QUICK, que apresentam vantagens e desvantagens características. A escolha do melhor método de interpolação é dependente do número de Peclet do processo:

$$
P_{e}=\frac{\rho \dot{R}(\phi, r, t) L}{\Gamma}
$$

O número de Peclet representa a razão entre as componentes convectiva e difusiva do escoamento ( $L$ é o comprimento característico). Comparações entre diferentes métodos de interpolação e critérios para sua escolha podem ser encontradas nos livros de Patankar (1980) e Versteeg e Malalasekera (1995).

Independentemente das interpolações escolhidas para os diferentes termos da equação diferencial, o resultado final do Método dos Volumes Finitos é um sistema de equações algébricas da forma:

$$
a_{P} \phi_{P}=a_{E} \phi_{E}+a_{W} \phi_{W}+b
$$

Sendo, para o equacionamento apresentado:

$$
\begin{aligned}
a_{E} & =\left[\frac{\Gamma}{\delta r}\right]_{e}-\frac{[\rho \dot{R}(\phi, r, t)]_{e}}{2} \\
a_{W} & =\left[\frac{\Gamma}{\delta r}\right]_{w}+\frac{[\rho \dot{R}(\phi, r, t)]_{w}}{2}
\end{aligned}
$$




$$
\begin{gathered}
a_{P}=\left[\frac{\Gamma}{\delta r}\right]_{e}+\frac{[\rho \dot{R}(\phi, r, t)]_{e}}{2}+\left[\frac{\Gamma}{\delta r}\right]_{w}-\frac{[\rho \dot{R}(\phi, r, t)]_{w}}{2}-S_{P} \Delta r \\
=a_{E}+a_{W}+[\rho \dot{R}(\phi, r, t)]_{e}-[\rho \dot{R}(\phi, r, t)]_{w}-S_{P} \Delta r \\
b=S_{C} \Delta r
\end{gathered}
$$

Se o fluido satisfizer a condição de continuidade, ou seja, se a vazão mássica de entrada no volume de controle for igual à de saída do mesmo (lembrando que o caso discutido é estacionário. Não há acúmulo nem consumo de massa), então:

$$
[\rho \dot{R}(\phi, r, t)]_{e}=[\rho \dot{R}(\phi, r, t)]_{w}
$$

E, de 2.1.13:

$$
a_{P}=a_{E}+a_{W}-S_{P} \Delta r
$$

Intuitivamente, para um sistema tri-dimensional:

$$
a_{P} \phi_{P}=a_{E} \phi_{E}+a_{W} \phi_{W}+a_{N} \phi_{N}+a_{S} \phi_{S}+a_{T} \phi_{T}+a_{B} \phi_{B}+b
$$

Sendo os subscritos $N, S, T$ e $B$ representativos dos pontos ao Norte, ao Sul, no topo e abaixo do ponto de referência, $P$, respectivamente.

O termo temporal, inicialmente omitido para simplificar o equacionamento, pode ser facilmente introduzido. Para um caso demonstrativo transiente, unidirecional, puramente difusivo, sem fonte e com as demais considerações anteriores:

$$
\frac{\partial[\rho \phi]}{\partial t}=\frac{\mathrm{d}}{\mathrm{d} r}\left[\Gamma \frac{\mathrm{d} \phi}{\mathrm{d} r}\right]
$$

Cuja forma integrada entre as interfaces do elemento de referência e no tempo é: 


$$
\int_{w}^{e} \int_{t}^{t+\Delta t} \frac{\partial[\rho \phi]}{\partial t} \mathrm{~d} t \mathrm{~d} r=\int_{w}^{e} \int_{t}^{t+\Delta t} \frac{\mathrm{d}}{\mathrm{d} r}\left[\Gamma \frac{\mathrm{d} \phi}{\mathrm{d} r}\right] \mathrm{d} t \mathrm{~d} r
$$

Assumindo que o valor $\phi_{P}$ prevalece ao redor de $P$ :

$$
\int_{w}^{e} \int_{t}^{t+\Delta t} \frac{\partial[\rho \phi]}{\partial t} \mathrm{~d} t \mathrm{~d} r=\rho \Delta r\left[\phi_{P}^{t+\Delta t}-\phi_{P}^{t}\right]
$$

Sendo $\phi_{P}^{t}$ e $\phi_{P}^{t+\Delta t}$ os valores de $\phi_{P}$ nos instantes $t$ e $t+\Delta t$, respectivamente.

Uma forma comum de integrar o lado direito da equação é a seguinte:

$$
\begin{aligned}
\int_{w}^{e} \int_{t}^{t+\Delta t} \frac{\mathrm{d}}{\mathrm{d} r}\left[\Gamma \frac{\mathrm{d} \phi}{\mathrm{d} r}\right] \mathrm{d} t \mathrm{~d} r=\int_{t}^{t+\Delta t}\left[\Gamma_{e} \frac{\left[\phi_{E}-\phi_{P}\right]}{[\delta r]_{e}}-\Gamma_{w} \frac{\left[\phi_{P}-\phi_{W}\right]}{[\delta r]_{w}}\right] \mathrm{d} t \\
=f\left[\Gamma_{e} \frac{\left[\phi_{E}^{t+\Delta t}-\phi_{P}^{t+\Delta t}\right]}{[\delta r]_{e}}-\Gamma_{w} \frac{\left[\phi_{P}^{t+\Delta t}-\phi_{W}^{t+\Delta t}\right]}{[\delta r]_{w}}\right] \\
+[1-f]\left[\Gamma_{e} \frac{\left[\phi_{E}^{t}-\phi_{P}^{t}\right]}{[\delta r]_{e}}-\Gamma_{w} \frac{\left[\phi_{P}^{t}-\phi_{W}^{t}\right]}{[\delta r]_{w}}\right]
\end{aligned}
$$

Sendo $f$ um fator entre 0 e 1 . $O$ valor dado a esse fator é o que diferencia os esquemas temporais Explícito $(f=0)$, Crank-Nicholson $(f=0,5)$ e Totalmente Implícito $(f=1)$. Suas características e limitações são descritas na literatura já mencionada do assunto.

O sistema de equações gerado é da forma (omitindo os sobrescritos $t+\Delta t \mathrm{e}$ substituindo os sobrescritos $t$ por 0 ):

$$
\begin{gathered}
a_{P} \phi_{P}=a_{E}\left[f \phi_{E}+[1-f] \phi_{E}^{0}\right]+a_{W}\left[f \phi_{W}+[1-f] \phi_{W}^{0}\right] \\
+\left[a_{P}^{0}-[1-f] a_{E}-[1-f] a_{W}\right] \phi_{P}^{0}
\end{gathered}
$$

Sendo:

$$
\begin{aligned}
a_{E} & =\left[\frac{\Gamma}{\delta r}\right]_{e} \\
a_{W} & =\left[\frac{\Gamma}{\delta r}\right]_{w}
\end{aligned}
$$




$$
\begin{gathered}
a_{P}^{0}=\frac{\rho_{P}^{0} \Delta r}{\Delta t} \\
a_{P}=f a_{E}+f a_{W}+a_{P}^{0}
\end{gathered}
$$

A escolha de um valor de $f$ permite uma simplificação maior do equacionamento.

Um comentário final importante sobre volumes finitos é a questão do acoplamento pressão-velocidade. No caso da resolução do campo de velocidades, tem-se $\phi=\dot{R}(r, t)$ e toda a formulação apresentada é válida. No entanto, se o campo de pressões não for previamente conhecido, o tratamento do termo fonte se complica. Se o fluido for contínuo, a utilização do campo de pressões correto no balanço de quantidade de movimento gera um campo de velocidades que satisfaz 0 balanço mássico do sistema. Uma solução iterativa se faz necessária, e vários algoritmos específicos para esse problema foram desenvolvidos, como o SIMPLE (Método Semi-Implícito para Equações Ligadas a Pressão) e suas variantes SIMPLER (SIMPLE Revisado) e SIMPLEC (SIMPLE Consistente) e o PISO (Pressão Implícita com Separação de Operadores), amplamente estudados e revisados na literatura.

\subsection{Escoamento Bifásico}

Escoamentos bifásicos (e multifásicos) diferem dos monofásicos pela existência de uma ou mais interfaces separando as fases ou componentes do sistema. Isso cria descontinuidades nas propriedades dos fluídos, tornando sua modelagem significativamente mais complexa que a tradicional de casos monofásicos. Ainda, essas interfaces não são estagnadas, demandando equacionamento próprio para descrever seu comportamento. Sistemas bifásicos podem ser modelados basicamente de duas formas: por formulação local instantânea e por formulação média no tempo (ISHII; HIBIKI, 2006).

$\mathrm{Na}$ formulação local instantânea, cada volume de fluido delimitado por uma ou mais interfaces é descrito com o equacionamento de sistemas monofásicos e as 
descontinuidades são equacionadas independentemente. As dificuldades matemáticas decorrentes de um modelo tão rigoroso fazem com que a aplicação desse método seja limitada a casos simples, como escoamentos separados ou quando o objetivo de estudo seja realmente o comportamento e os transportes ocorridos nas interfaces. Essa formulação também é a base do equacionamento médio.

Na formulação média no tempo é buscada uma simplificação análoga àquela que possibilita a descrição do fenômeno da turbulência: em troca da perda das informações locais, espera-se possibilitar a descrição do sistema como um todo. É impossível equacionar o sistema inteiro sem violar a condição de continuidade do equacionamento de transporte tradicional, mas, sabendo que cada ponto no espaço é ocupado, aleatoriamente, por uma das duas fases do sistema alternadamente, é possível derivar um equacionamento médio (no tempo) análogo ao de casos monofásicos. Os transportes ocorridos nas interfaces, que antes tinham equacionamento próprio, são substituídos por termos de interação nos balanços de cada fase do sistema e as interfaces em si são equacionadas estatisticamente. Essa aproximação é, por sua simplicidade, útil para a descrição de sistemas multifásicos e dispersos.

A formulação euleriana mediada no tempo, por sua vez, dá origem a dois modelos: o modelo de mistura e o modelo bifásico. O modelo de mistura se baseia em descrever ambas as fases como uma só. São escritas as equações de transporte da mistura (massa, energia e quantidade de movimento) e uma equação de difusão, responsável pela variação na concentração da mesma. Ele provê uma descrição das propriedades e do comportamento da mistura, mas não sobre a interação e o comportamento dos diferentes componentes. Já o modelo bifásico origina um conjunto de equações para cada fase, relacionadas entre si por termos de interação, e uma equação para descrever a distribuição da fase dispersa, o balanço populacional. Este é mais complexo que o modelo de mistura, mas é mais adequado para descrever sistemas em que as fases diferem em comportamento, como com velocidades de escoamento distintas. 


\section{Modelo Bifásico}

O desenvolvimento do modelo bifásico é mostrado por Gidaspow (1994) e desenvolvido com preciosismo por Ishii e Hibiki (2006). O equacionamento mostrado por ambos é equivalente. Tem-se o balanço de massa de cada fase $\beta$ :

$$
\frac{\partial\left[\gamma_{\beta} \rho_{\beta}\right]}{\partial t}+\nabla \cdot\left[\gamma_{\beta} \rho_{\beta} \dot{\boldsymbol{R}}(\boldsymbol{r}, t)\right]=Z_{\beta}
$$

Com

$$
\sum_{\beta=1}^{2} Z_{\beta}=0
$$

Sendo $\gamma_{\beta}$ a fração volumétrica da fase $\beta, \rho_{\beta}$ a sua massa específica média, $\dot{\boldsymbol{R}}(r, t)$ o campo de velocidades bulk ao qual está submetida e $Z_{\beta}$ o termo de transferência interfacial mássico entre ela e a outra fase.

$O$ balanço de quantidade de movimento da fase $\beta$ é equacionado:

$$
\frac{\partial\left[\gamma_{\beta} \rho_{\beta} \dot{\boldsymbol{R}}(\boldsymbol{r}, t)\right]}{\partial t}+\nabla \cdot\left[\gamma_{\beta} \rho_{\beta} \dot{\boldsymbol{R}}(\boldsymbol{r}, t) \dot{\boldsymbol{R}}(\boldsymbol{r}, t)\right]=\nabla \cdot\left[\gamma_{\beta} \boldsymbol{T}_{\beta}\right]+\gamma_{\beta} \rho_{\beta} \boldsymbol{g}+\boldsymbol{M}_{\beta}
$$

Sendo $\boldsymbol{T}_{\beta}$ é o tensor efetivo da fase $\beta$, composto das componentes viscosidade e turbulência do equacionamento, $\boldsymbol{g}$ ○ campo gravitacional ao qual o fluído está submetido e $\boldsymbol{M}_{\beta}$ o termo de transferência interfacial de quantidade de movimento entre as fases.

Discussão extensa desse equacionamento e do balanço de energia pode ser encontrada no livro de Ishii e Hibiki (2006) e Silva; Damian e Lage (2008) mostram um exemplo de aplicação.

$O$ termos de transferência, $Z_{\beta}$ e $\boldsymbol{M}_{\beta}$ dependem do conhecimento da área interfacial entre as fases. Para os casos em que essas descontinuidades não são fixas como, por exemplo, sistemas polidispersos líquido-líquido, nos quais há uma fase contínua e uma dispersa com uma grande distribuição de tamanho de gotas, 
faz-se necessário um modelo que permita acompanhar e prever o comportamento das mesmas. Dessa forma, a modelagem de tal sistema exige a solução não só dos balanços apresentados mas, também, do balanço de número de gotas, ou balanço populacional, da fase dispersa.

\subsection{Equação do Balanço Populacional (EBP)}

A modelagem mostrada a seguir é deduzida de acordo com os princípios e considerações de Ramkrishna (2000).

Dado um sistema bifásico onde há distinção clara entre uma fase contínua e uma dispersa, é possível agrupar as coordenadas que definem o comportamento de uma partícula no sistema em externas $\boldsymbol{r} \equiv\left(r_{1}, r_{2}, r_{3}\right)$, que definem a posição de seu centro de massa, e internas $\boldsymbol{x} \equiv\left(x_{1}, x_{2}, \ldots, x_{d}\right)$, representando $d$ variáveis associadas à mesma, como massa e temperatura. $\boldsymbol{\Omega}_{r}$ e $\boldsymbol{\Omega}_{x}$ são os domínios das coordenadas externas e internas, respectivamente.

Ainda, define-se $\boldsymbol{Y} \equiv\left[Y_{1}(\boldsymbol{r}, t), Y_{2}(\boldsymbol{r}, t), \ldots, Y_{c}(\boldsymbol{r}, t)\right]$ como o vetor de variáveis da fase contínua que afetam o comportamento de cada partícula, variáveis estas governadas por leis de transporte e interação com o particulado.

De posse dessas informações, define-se a função densidade numérica média de partículas $f(\boldsymbol{x}, \boldsymbol{r}, t)$, que permite representar o número médio de partículas no hiper-volume infinitesimal $\mathrm{d} V_{x} \mathrm{~d} V_{\boldsymbol{r}}$ ao redor de $(\boldsymbol{x}, \boldsymbol{r})$ por $f(\boldsymbol{x}, \boldsymbol{r}, t) \mathrm{d} V_{\boldsymbol{x}} \mathrm{d} V_{\boldsymbol{r}}$. Outras informações relevantes decorrem imediatamente dessa definição, como o número total (esperado) de partículas em um instante $t$ por unidade de volume físico $N(\boldsymbol{r}, t)$ :

$$
N(\boldsymbol{r}, t)=\int_{\Omega_{x}} f(\boldsymbol{x}, \boldsymbol{r}, t) \mathrm{d} V_{\boldsymbol{x}}
$$

Da mesma forma, é possível obter a fração volumétrica total da fase dispersa $\gamma_{d}(\boldsymbol{r}, t)$. Sendo $v(\boldsymbol{x})$ o volume da partícula de estado (interno) $\boldsymbol{x}$, a densidade volumétrica de partículas no espaço físico é $v(\boldsymbol{x}) f(\boldsymbol{x}, \boldsymbol{r}, t)$. Logo: 


$$
\gamma_{d}(\boldsymbol{r}, t)=\int_{\Omega_{x}} v(\boldsymbol{x}) f(\boldsymbol{x}, \boldsymbol{r}, t) \mathrm{d} V_{x}
$$

Voltando à discussão da EBP, como $f(\boldsymbol{x}, \boldsymbol{r}, t)$ representa a quantidade de partículas por unidade de volume, pode-se escrever um balanço microscópico euleriano de partículas no volume de controle $\mathrm{d} V_{x} \mathrm{~d} V_{r}$ como:

$$
\begin{aligned}
\frac{\partial f(\boldsymbol{x}, \boldsymbol{r}, t)}{\partial t}+\nabla_{\mathbf{x}} \cdot[\dot{\boldsymbol{X}}(\boldsymbol{x}, \boldsymbol{r}, \boldsymbol{Y}, t) f(\boldsymbol{x}, \boldsymbol{r}, t)]+\nabla_{\mathbf{r}} \cdot[\dot{\boldsymbol{R}}(\boldsymbol{x}, \boldsymbol{r}, \boldsymbol{Y}, t) f(\boldsymbol{x}, \boldsymbol{r}, t)] \\
=-\nabla \cdot \boldsymbol{j}(\boldsymbol{x}, \boldsymbol{r}, \boldsymbol{Y}, t)+S(\boldsymbol{x}, \boldsymbol{r}, \boldsymbol{Y}, t)
\end{aligned}
$$

Sendo $\dot{\boldsymbol{X}}(\boldsymbol{x}, \boldsymbol{r}, \boldsymbol{Y}, t)$ e $\dot{\boldsymbol{R}}(\boldsymbol{x}, \boldsymbol{r}, \boldsymbol{Y}, t)$ velocidades determinísticas às quais está submetida a população, relativas às coordenadas internas e externas, respectivamente. $\boldsymbol{j}(\boldsymbol{x}, \boldsymbol{r}, \boldsymbol{Y}, t)$ é o termo difusivo que, por se tratar de um equacionamento médio, é associado a mudanças aleatórias no estado das partículas do sistema, e $S(\boldsymbol{x}, \boldsymbol{r}, \boldsymbol{Y}, t)$ é o termo fonte.

Para sistemas com comportamento puramente determinístico, ou cujo comportamento aleatório seja desprezível, $\boldsymbol{j}(\boldsymbol{x}, \boldsymbol{r}, \boldsymbol{Y}, t)$ é desconsiderado (DAMIAN, 2007). O campo $\dot{X}(\boldsymbol{x}, \boldsymbol{r}, \boldsymbol{Y}, t)$ determina o deslocamento da população pelo espaço de coordenadas internas, ou seja, a velocidade de crescimento da partícula (como, por exemplo, em processos de cristalização). É comum na literatura o agrupamento deste termo com $S(\boldsymbol{x}, \boldsymbol{r}, \boldsymbol{Y}, t)$ quando há conveniência em descrever separadamente a evolução de $f(\boldsymbol{x}, \boldsymbol{r}, t)$ no domínio das coordenadas internas e externas (RAMKRISHNA, 2000; CAMPOS; LAGE, 2003; MARCHISIO; FOX, 2005; SU et al., 2008). Vale observar também que $\dot{\boldsymbol{R}}(\boldsymbol{x}, \boldsymbol{r}, \boldsymbol{Y}, t)$ não é nada mais que o campo de velocidades ao qual estão submetidas as partículas. Em sistemas com uma fase contínua e somente uma dispersa, este termo representa a velocidade (média) da última. Finalmente, para sistemas em que agregação e quebra podem ser considerados ocorrendo independentemente, é conveniente decompor o termo fonte do balanço em nascimento $(B)$ e morte $(D)$ de partículas pelo primeiro (sobrescrito $A$ ) e pelo segundo fenômenos (sobrescrito $B$ ). Assim:

$$
S(\boldsymbol{x}, \boldsymbol{r}, \boldsymbol{Y}, t)=B^{A}(\boldsymbol{x}, \boldsymbol{r}, \boldsymbol{Y}, t)+B^{B}(\boldsymbol{x}, \boldsymbol{r}, \boldsymbol{Y}, t)-D^{A}(\boldsymbol{x}, \boldsymbol{r}, \boldsymbol{Y}, t)-D^{B}(\boldsymbol{x}, \boldsymbol{r}, \boldsymbol{Y}, t) \quad 2.3 .4
$$


O que resulta na seguinte equação simplificada para processos de agregação e quebra sem crescimento:

$$
\begin{aligned}
\frac{\partial f(\boldsymbol{x}, \boldsymbol{r}, t)}{\partial t}+\nabla_{\mathbf{r}} & \cdot[\dot{\boldsymbol{R}}(\boldsymbol{x}, \boldsymbol{r}, \boldsymbol{Y}, t) f(\boldsymbol{x}, \boldsymbol{r}, t)] \\
& =B^{A}(\boldsymbol{x}, \boldsymbol{r}, \boldsymbol{Y}, t)+B^{B}(\boldsymbol{x}, \boldsymbol{r}, \boldsymbol{Y}, t)-D^{A}(\boldsymbol{x}, \boldsymbol{r}, \boldsymbol{Y}, t) \\
& -D^{B}(\boldsymbol{x}, \boldsymbol{r}, \boldsymbol{Y}, t)
\end{aligned}
$$

\subsubsection{Agregação}

Considera-se o sistema diluído o suficiente para que a freqüência de agregação simultânea de múltiplas partículas (originando uma única partícula) seja desconsiderável frente à de somente duas. Partindo desse princípio, faz-se possível definir a fração de partículas de estado $(\boldsymbol{x}, \boldsymbol{r})$ e $\left(\boldsymbol{x}^{\prime}, \boldsymbol{r}\right)$ sofrendo agregação por unidade de tempo (ou sua probabilidade de agregação) como $a\left(\boldsymbol{x}, \boldsymbol{r} ; \boldsymbol{x}^{\prime}, \boldsymbol{r}^{\prime} ; \boldsymbol{Y}, t\right)$, sendo que, por simetria:

$$
a\left(\boldsymbol{x}, \boldsymbol{r} ; \boldsymbol{x}^{\prime}, \boldsymbol{r}^{\prime} ; \boldsymbol{Y}, t\right)=a\left(\boldsymbol{x}^{\prime}, \boldsymbol{r}^{\prime} ; \boldsymbol{x}, \boldsymbol{r} ; \boldsymbol{Y}, t\right)
$$

É necessário saber a quantidade de pares de partículas cuja frequência de agregação é $a\left(\boldsymbol{x}, \boldsymbol{r} ; \boldsymbol{x}^{\prime}, \boldsymbol{r}^{\prime} ; \boldsymbol{Y}, t\right)$. Define-se então $f_{2}\left(\boldsymbol{x}^{\prime}, \boldsymbol{r}^{\prime} ; \boldsymbol{x}, \boldsymbol{r} ; t\right)$ como a função densidade numérica média de pares de partículas distintos ao redor de $\left(\boldsymbol{x}^{\prime}, \boldsymbol{r}\right)$ e $(\boldsymbol{x}, \boldsymbol{r})$ num instante $t$.

Tomando uma nova partícula de estado $\left(\boldsymbol{x}^{\prime \prime}, \boldsymbol{r}^{\prime \prime}\right)$, os termos de agregação do balanço podem ser escritos como:

$$
\begin{aligned}
& D^{A}(\boldsymbol{x}, \boldsymbol{r}, \boldsymbol{Y}, t)=\int_{\Omega_{x}} \int_{\Omega_{r}} a\left(\boldsymbol{x}^{\prime}, \boldsymbol{r}^{\prime} ; \boldsymbol{x}, \boldsymbol{r} ; \boldsymbol{Y}, t\right) f_{2}\left(\boldsymbol{x}^{\prime}, \boldsymbol{r}^{\prime} ; \boldsymbol{x}, \boldsymbol{r} ; t\right) \mathrm{d} V_{\boldsymbol{r}^{\prime}} \mathrm{d} V_{\boldsymbol{x}^{\prime}} \\
& B^{A}(\boldsymbol{x}, \boldsymbol{r}, \boldsymbol{Y}, t) \\
& =\int_{\boldsymbol{\Omega}_{x}} \int_{\Omega_{r}} \frac{1}{\delta} a\left(\boldsymbol{x}^{\prime \prime}, \boldsymbol{r}^{\prime \prime} ; \boldsymbol{x}^{\prime}, \boldsymbol{r}^{\prime} ; \boldsymbol{Y}, t\right) f_{2}\left(\boldsymbol{x}^{\prime \prime}, \boldsymbol{r}^{\prime \prime} ; \boldsymbol{x}^{\prime}, \boldsymbol{r}^{\prime} ; t\right) \frac{\partial\left(\boldsymbol{x}^{\prime \prime}, \boldsymbol{r}^{\prime \prime}\right)}{\partial(\boldsymbol{x}, \boldsymbol{r})} \mathrm{d} V_{\boldsymbol{r}^{\prime}} \mathrm{d} V_{\boldsymbol{x}^{\prime}}
\end{aligned}
$$


Sendo $\frac{\partial\left(x^{\prime \prime}, r^{\prime \prime}\right)}{\partial(x, r)}$ ○ Jacobiano da transformação de coordenadas do espaço $\left(x^{\prime \prime}, \boldsymbol{r}^{\prime \prime}\right)$ para ○ $(\boldsymbol{x}, \boldsymbol{r})$ e $\delta$ o número de vezes que pares idênticos de partículas são considerados $\left(\frac{1}{\delta}\right.$ corrige a redundância).

Os termos apresentados não são fechados. Analogamente ao fato de que a descrição de $f(\boldsymbol{x}, \boldsymbol{r}, t)$ necessitou da definição de $f_{2}\left(\boldsymbol{x}^{\prime}, \boldsymbol{r}^{\prime} ; \boldsymbol{x}, \boldsymbol{r} ; t\right)$, uma equação que descrevesse $f_{2}\left(\boldsymbol{x}^{\prime}, \boldsymbol{r}^{\prime} ; \boldsymbol{x}, \boldsymbol{r} ; t\right)$ envolveria uma terceira função, $f_{3}$, e, sucessivamente, infinitas incógnitas. Dessa maneira, é adotada comumente a seguinte aproximação:

$$
f_{2}\left(\boldsymbol{x}^{\prime}, \boldsymbol{r}^{\prime} ; \boldsymbol{x}, \boldsymbol{r} ; t\right)=f\left(\boldsymbol{x}^{\prime}, \boldsymbol{r}^{\prime}, t\right) f(\boldsymbol{x}, \boldsymbol{r}, t)
$$

Que basicamente desconsidera qualquer relação estatística entre as partículas em $\left(\boldsymbol{x}^{\prime}, \boldsymbol{r}\right)$ e $(\boldsymbol{x}, \boldsymbol{r})$, ou seja, considera uma probabilidade igual de supostas partículas em qualquer estado formarem pares, por mais improvável que seja a sua existência ou proximidade. O erro associado a essa relação é inversamente proporcional ao tamanho da população em questão.

\section{O Caso Monovariável}

É comum, por simplicidade matemática ou do sistema em si, utilizar-se de somente uma coordenada interna na EBP. Para o caso particular de uma população de partículas distribuída de acordo com as suas respectivas massas tem-se, por conservação, se uma partícula de massa $x "$ se agrega a outra de massa $x$ ' para formar uma de massa $x$ :

$$
x^{\prime \prime}=x-x^{\prime}
$$

Dessa relação, tratando-se de um sistema de coordenadas externas ortonormal, é possível observar que o Jacobiano da transformada de coordenadas se reduz a: 


$$
\frac{\partial\left(\boldsymbol{x}^{\prime \prime}, \boldsymbol{r}^{\prime \prime}\right)}{\partial(\boldsymbol{x}, \boldsymbol{r})}=\frac{\partial x^{\prime \prime}}{\partial x}=1
$$

Além disso, como

$$
0<x^{\prime}<x
$$

$E$

$$
0<x^{\prime \prime}=x-x^{\prime}<x
$$

Vê-se que cada par $\left\{x^{\prime}, x^{\prime \prime}\right\}$ é considerado duas vezes, ou seja, $\delta=2$.

Para o caso em que não haja dependência espacial direta do fenômeno de agregação (só indireta por meio de $\boldsymbol{Y}$ ), os termos de sua modelagem podem, então, ser reescritos como:

$$
\begin{gathered}
D^{A}(x, \boldsymbol{Y}, t)=f(x, t) \int_{0}^{\infty} a\left(x^{\prime} ; x ; \boldsymbol{Y}, t\right) f\left(x^{\prime}, t\right) \mathrm{d} x^{\prime} \\
B^{A}(x, \boldsymbol{Y}, t)=\frac{1}{2} \int_{0}^{\infty} a\left(x^{\prime \prime} ; x^{\prime} ; \boldsymbol{Y}, t\right) f\left(x^{\prime \prime}, t\right) f\left(x^{\prime}, t\right) \mathrm{d} x^{\prime \prime}
\end{gathered}
$$

\subsubsection{Quebra}

Analogamente ao processo de agregação de partículas, define-se a fração de partículas de estado $(\boldsymbol{x}, \boldsymbol{r})$ sofrendo quebra por unidade de tempo (ou sua freqüência de quebra) como $b(\boldsymbol{x}, \boldsymbol{r}, \boldsymbol{Y}, t)$. É deduzido diretamente que:

$$
D^{B}(\boldsymbol{x}, \boldsymbol{r}, \boldsymbol{Y}, t)=b(\boldsymbol{x}, \boldsymbol{r}, \boldsymbol{Y}, t) f(\boldsymbol{x}, \boldsymbol{r}, t)
$$

A dedução do termo de nascimento de partículas por quebra requer a introdução de duas novas definições: o número médio de partículas originadas da quebra de uma única partícula de estado $(\boldsymbol{x}, \boldsymbol{r}), \vartheta(\boldsymbol{x}, \boldsymbol{r}, \boldsymbol{Y}, t)$, e a função densidade de probabilidade de partículas originadas da quebra de uma partícula de estado $\left(\boldsymbol{x}^{\prime}, \boldsymbol{r}\right)$ 
terem o estado $(\boldsymbol{x}, \boldsymbol{r}), P\left(\boldsymbol{x}, \boldsymbol{r} \mid \boldsymbol{x}^{\prime}, \boldsymbol{r}^{\prime}, \boldsymbol{Y}, t\right)$. A primeira é restrita, logicamente, por $\vartheta(\boldsymbol{x}, \boldsymbol{r}, \boldsymbol{Y}, t) \geq 2$, mas pode adquirir valores reais sendo uma quantidade média. A distribuição $P\left(\boldsymbol{x}, \boldsymbol{r} \mid \boldsymbol{x}^{\prime}, \boldsymbol{r}^{\prime}, \boldsymbol{Y}, t\right)$ precisa satisfazer as condições de normalização e conservação de massa, respectivamente:

$$
\begin{gathered}
\int_{\boldsymbol{\Omega}_{x}} P\left(\boldsymbol{x}, \boldsymbol{r} \mid \boldsymbol{x}^{\prime}, \boldsymbol{r}^{\prime}, \boldsymbol{Y}, t\right) \mathrm{d} V_{\boldsymbol{x}}=1 \\
P\left(\boldsymbol{x}, \boldsymbol{r} \mid \boldsymbol{x}^{\prime}, \boldsymbol{r}^{\prime}, \boldsymbol{Y}, t\right)=0, m(\boldsymbol{x}) \geq m(\boldsymbol{x})
\end{gathered}
$$

Sendo $m(\boldsymbol{x})$ a massa de uma partícula de estado interno $\boldsymbol{x}$.

Outra exigência imposta pelo princípio da conservação de massa no processo é:

$$
m(\boldsymbol{x}) \geq \vartheta\left(\boldsymbol{x}^{\prime}, \boldsymbol{r}^{\prime}, \boldsymbol{Y}, t\right) \int_{\boldsymbol{\Omega}_{x}} m(\boldsymbol{x}) P\left(\boldsymbol{x}, \boldsymbol{r} \mid \boldsymbol{x}^{\prime}, \boldsymbol{r}^{\prime}, \boldsymbol{Y}, t\right) \mathrm{d} V_{\boldsymbol{x}}
$$

Sendo o caso em que não há perda de massa pelo processo de quebra representado pela igualdade entre os termos à esquerda e à direita.

Com isso, é possível escrever o termo que representa o nascimento de partículas de estado $(\boldsymbol{x}, \boldsymbol{r})$ pela quebra de partículas de qualquer outro estado em $\Omega_{r} \times \Omega_{x}$ :

$$
\begin{aligned}
& B^{B}(\boldsymbol{x}, \boldsymbol{r}, \boldsymbol{Y}, t) \\
& =\int_{\boldsymbol{\Omega}_{x}} \int_{\boldsymbol{\Omega}_{\boldsymbol{r}}} \vartheta\left(\boldsymbol{x}^{\prime}, \boldsymbol{r}^{\prime}, \boldsymbol{Y}, t\right) b\left(\boldsymbol{x}^{\prime}, \boldsymbol{r}^{\prime}, \boldsymbol{Y}, t\right) P\left(\boldsymbol{x}, \boldsymbol{r} \mid \boldsymbol{x}^{\prime}, \boldsymbol{r}^{\prime}, \boldsymbol{Y}, t\right) f\left(\boldsymbol{x}^{\prime}, \boldsymbol{r}^{\prime}, t\right) \mathrm{d} V_{\boldsymbol{r}^{\prime}} \mathrm{d} V_{\boldsymbol{x}^{\prime}}
\end{aligned}
$$

\section{O Caso Monovariável}

Tomando novamente a massa das partículas como a única coordenada interna e, ainda, assumindo um comportamento binário para o fenômeno, temos:

$$
\vartheta \equiv 2
$$


E, por simetria:

$$
P\left(x, \boldsymbol{r} \mid x^{\prime}, \boldsymbol{r}^{\prime}, \boldsymbol{Y}, t\right)=P\left(x^{\prime}-x, \boldsymbol{r} \mid x^{\prime}, \boldsymbol{r}^{\prime}, \boldsymbol{Y}, t\right)
$$

Ou seja, a probabilidade de uma partícula de massa $x^{\prime}$ se quebrar originando uma de massa $x$ deve ser igual à de originar a sua complementar de massa $x^{\prime}-x$.

Considerando novamente independência espacial direta, a forma final dos termos de quebra para esse caso é, então:

$$
\begin{gathered}
D^{B}(x, \boldsymbol{Y}, t)=b(x, \boldsymbol{Y}, t) f(x, t) \\
B^{B}(x, \boldsymbol{Y}, t)=2 \int_{x}^{\infty} b\left(x^{\prime}, \boldsymbol{Y}, t\right) P\left(x \mid x^{\prime}, \boldsymbol{Y}, t\right) f\left(x^{\prime}, t\right) \mathrm{d} x^{\prime}
\end{gathered}
$$

\subsection{Métodos Numéricos para a Resolução de Balanços Populacionais}

A EBP (equação 2.3.3) é, usualmente, intratável (FRENKLACH; HARRIS, 1987; RAMKRISHNA; MAHONEY, 2002). No entanto, devido à sua importância para descrever sistemas dispersos, o desenvolvimento de métodos numéricos que possibilitem sua resolução tem sido objeto de estudo desde meados da segunda metade do século passado (HULBURT; KATZ, 1964; KUMAR; RAMKRISHNA, 1996; MCGRAW, 1997), excepcionalmente durante a presente década (FAN; MARCHISIO; FOX, 2004; BOVE; SOLBERG; HJERTAGER, 2005; ATTARAKIH; BART; FAQIR, 2006; KUMAR et al., 2006; SU et al., 2007, 2008; ATTARAKIH; DRUMM; BART, 2009; GU et al., 2009). O Método dos Momentos (MOM) de Hulburt e Katz (1964) foi o primeiro trabalho a se focar especificamente na resolução numérica da EBP (SU; GU; XU, 2009).

Seguindo essa linha, o método em maior evidência atualmente é, muito provavelmente, o Método Direto das Quadraturas dos Momentos (DQMOM) de Fan; Marchisio e Fox (2004), que é uma implementação diferente do Método das Quadraturas dos Momentos (QMOM) de McGraw (1997). O DQMOM é computacionalmente mais eficiente que o QMOM, mas é mais sensível a erros 
numéricos (MARCHISIO; FOX, 2005). Apesar de ser relativamente recente, já existem muitos trabalhos que estudam sua implementação e acoplamento com simulações CFD (FOX, 2006; ZUCCA et al., 2006; SILVA; DAMIAN; LAGE 2008; FOX; LAURENT; MASSOT, 2008; SU et al., 2008). Ambos os métodos não requerem um conhecimento completo da distribuição de partículas inicial do sistema, somente de alguns de seus momentos, o que facilita seu equacionamento. Por essas razões, o QMOM e o DQMOM foram escolhidos como os métodos a serem explorados neste estudo, e a revisão que segue visa explicitar seu equacionamento e as idéias nas quais eles se baseiam.

\subsubsection{Método dos Momentos (MOM)}

Introduzido por Hulburt e Katz (1964) para sistemas descritos por somente uma coordenada interna, consiste em substituir a resolução do balanço da função densidade numérica média de partículas pela do de um conjunto de momentos de baixa ordem, tidos como suficientes para descrever as propriedades relevantes do sistema.

Sendo o momento de ordem $k$ definido como:

$$
m_{k}(\boldsymbol{r}, t)=\int_{\Omega_{x}} x^{k} f(x, \boldsymbol{r}, t) \mathrm{d} x
$$

E a EBP da forma:

$$
\frac{\partial f(x, \boldsymbol{r}, t)}{\partial t}+\nabla_{\mathbf{r}} \cdot[\dot{\boldsymbol{R}}(\boldsymbol{r}, \boldsymbol{Y}, t) f(x, \boldsymbol{r}, t)]=-\nabla \cdot \boldsymbol{j}(x, \boldsymbol{r}, \boldsymbol{Y}, t)+S(x, \boldsymbol{r}, \boldsymbol{Y}, t)
$$

Com o termo de deslocamento da partícula pelo campo da coordenada interna (termo de crescimento) combinado ao termo fonte, multiplicando-a por $x^{k} \mathrm{e}$ integrando a equação em $x$ por todo o domínio $\Omega_{x}$ :

$$
\frac{\partial m_{k}(\boldsymbol{r}, t)}{\partial t}+\nabla_{\mathbf{r}} \cdot\left[\dot{\boldsymbol{R}}(\boldsymbol{r}, \boldsymbol{Y}, t) m_{k}(\boldsymbol{r}, t)\right]=-\nabla \cdot \boldsymbol{j}_{k}(\boldsymbol{r}, \boldsymbol{Y}, t)+S_{k}(\boldsymbol{r}, \boldsymbol{Y}, t)
$$


Que representa o sistema de $k$ equações diferenciais nas quais se baseia o método.

A grande vantagem dessa abordagem do problema do balanço populacional, além de se utilizar de um número reduzido de escalares, é não ser necessário um conhecimento profundo da distribuição de partículas em si, só da evolução dos seus momentos, grandezas essas com significados físicos bem definidos e que, em muitos casos, são exatamente as informações que se deseja obter do sistema. Por exemplo, o momento de ordem zero representa a integração da própria função densidade numérica média de partículas $f(x, r, t)$ por todo o espaço de coordenadas internas (a densidade numérica média de partículas em sua totalidade) e o de primeira ordem, para uma população definida pela massa de suas partículas, a densidade mássica da fase dispersa.

Apesar de facilitar o tratamento de sistemas dispersos, o Método dos Momentos tem duas severas limitações:

- Para que a equação 2.3.1.3 seja verdadeira, o termo $\dot{\boldsymbol{R}}(\boldsymbol{r}, \boldsymbol{Y}, t)$ deve ser independente da coordenada interna $x$. Ou seja, todas as partículas distribuídas em $\Omega_{x}$ por $x$ devem compartilhar o mesmo campo de velocidades. Essa consideração limita a aplicação do método a populações para as quais haja distribuição concentrada ao redor de um ponto em $x$ ou cuja coordenada interna seja escolhida de forma a não influenciar significativamente esse campo.

- O sistema de equações diferenciais resultante (mais especificamente o termo fonte $\left.S_{k}(\boldsymbol{r}, \boldsymbol{Y}, t)\right)$ só é fechado para alguns casos simples. De forma geral, a evolução do $k$-ésimo momento depende de um momento de ordem maior, o que cria a necessidade de uma formulação para o fechamento específica para cada problema, limitando, assim, sua aplicabilidade (MCGRAW, 1997). Apesar disso, Wan e Ring (2006) mostraram que seu acoplamento com CFD é possível, se restrito.

\subsubsection{Método das Quadraturas dos Momentos (QMOM)}

Tomando, por exemplo, o problema apresentado por Hulburt e Katz, 1964 para nucleação e crescimento de partículas: 


$$
\begin{aligned}
& S_{k}(\boldsymbol{r}, \boldsymbol{Y}, t)= n(\boldsymbol{r}, \boldsymbol{Y}, t) \int_{\Omega_{x}} x^{k} \psi(x) \mathrm{d} x \\
&+g(\boldsymbol{r}, \boldsymbol{Y}, t) k \int_{\Omega_{x}} x^{k-1} \phi(x) f(x, \boldsymbol{r}, t) \mathrm{d} x \\
& N(x, \boldsymbol{r}, \boldsymbol{Y}, t)=n(\boldsymbol{r}, \boldsymbol{Y}, t) \psi(x) \\
& G(x, \boldsymbol{r}, \boldsymbol{Y}, t)=g(\boldsymbol{r}, \boldsymbol{Y}, t) \phi(x)
\end{aligned}
$$

Sendo $N(x, \boldsymbol{r}, \boldsymbol{Y}, t)$ e $G(x, \boldsymbol{r}, \boldsymbol{Y}, t)$ as funções de nucleação e crescimento de partículas, respectivamente. O fechamento exato do segundo termo à direita da equação 2.4.2.1 só é obtido se $\phi(x)$ for da forma:

$$
\phi(x)=\beta_{0}+\beta_{1} x
$$

O que resulta em:

$$
\begin{aligned}
k g(\boldsymbol{r}, \boldsymbol{Y}, t) \int_{\Omega_{x}} x^{k-1} \phi(x) f(x, \boldsymbol{r}, t) \mathrm{d} x \\
\quad=k g(\boldsymbol{r}, \boldsymbol{Y}, t)\left[\beta_{0} m_{k-1}(\boldsymbol{r}, t)+\beta_{1} m_{k}(\boldsymbol{r}, t)\right]
\end{aligned}
$$

E cada momento $m_{k}(\boldsymbol{r}, t)$ for dependente somente de momentos de ordens menores. Se esse comportamento não for observado, o fechamento não é obtido. Em vista do potencial do Método dos Momentos e ciente de suas limitações, McGraw (1997) propôs uma extensão do mesmo, o Método das Quadraturas dos Momentos, que elimina a necessidade de fechamento exato do sistema ao introduzir um fechamento aproximado dos termos problemáticos por meio de uma quadratura gaussiana de $N$ pontos. Para o caso em questão:

$$
\int_{\Omega_{x}} x^{k-1} \phi(x) f(x, \boldsymbol{r}, t) \mathrm{d} x \cong \sum_{\alpha=1}^{N} x_{\alpha}{ }^{k-1}(\boldsymbol{r}, t) \phi\left(x_{\alpha}(\boldsymbol{r}, t)\right) \omega_{\alpha}(\boldsymbol{r}, t)
$$


Sendo que os $N$ pares de pesos $\omega_{\alpha}$ e abscissas $x_{\alpha}$ podem ser determinados pela aplicação do algoritmo Produto-Diferença (GORDON, 1968) ao seguinte sistema de equações:

$$
m_{k}=\int_{\Omega_{x}} x^{k} f(x, \boldsymbol{r}, t) \mathrm{d} x \cong \sum_{\alpha=1}^{N} \omega_{\alpha}(\boldsymbol{r}, t) x_{\alpha}{ }^{k}(\boldsymbol{r}, t), k=0, \ldots, 2 N-1
$$

Que é obtido diretamente da aproximação por quadratura gaussiana previamente introduzida. A idéia da aproximação por quadratura pode ser melhor entendida graficamente, como mostrado na figura 2.4.2.1.

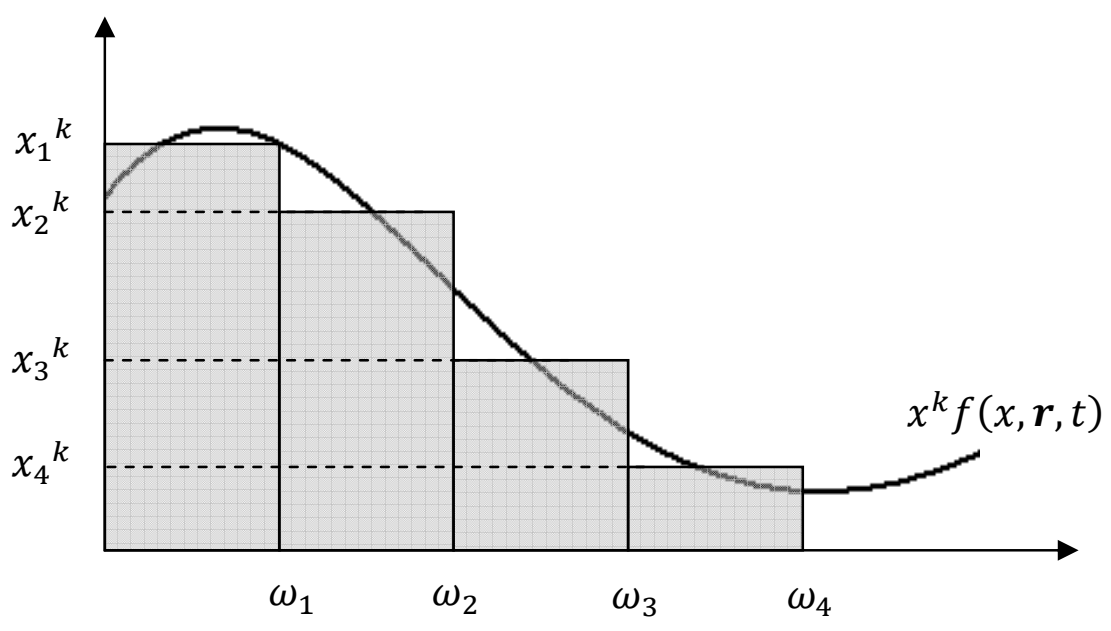

Figura 2.4.2.1 - Representação gráfica da aproximação por quadratura

Além do caso de nucleação e crescimento de partículas para o qual foi inicialmente proposto, o método foi estendido e validado para agregação (BARRET; WEBB, 1998; MARCHISIO et al., 2003b), duas coordenadas internas (WRIGHT; MCGRAW; ROSNER, 2001) e quebra (MARCHISIO; VIRGIL; FOX, 2003a). A possibilidade de sua implementação em códigos CFD foi comprovada (MARCHISIO; VIRGIL; FOX, 2003c; DRUMM; ATTARAKIH; BART, 2009), e seus resultados se mostram equivalentes aos obtidos pelo MOM original, mesmo para os limitados casos em que ele pode ser aplicado (WAN; RING, 2006).

Apesar dos avanços apresentados, O QMOM não é livre de restrições. O problema do fechamento do MOM foi contornado, mas sua extensão para casos com mais de uma coordenada interna considerada retém as limitações do mesmo. Ainda, o algoritmo PD (GORDON, 1968), utilizado para calcular os pesos e 
abscissas da quadratura (e que, num acoplamento CFD, precisa ser recalculado em cada ponto da malha e a cada passo temporal), perde sua simplicidade para casos multivariados (WRIGHT; MCGRAW; ROSNER, 2001). O caso multivariado torna-se ainda mais dispendioso matematicamente que no MOM. Finalmente, Fan; Marchisio e Fox (2004) apontam a impropriedade do método para sistemas com escoamentos multifásicos (em contraste com bifásicos), uma vez que ele retém as equações de transporte de momentos originais do $\mathrm{MOM}$, nas quais a velocidade da fase dispersa é tida como invariante na coordenada interna. Sua extensão para a resolução de mais de uma fase dispersa envolve, como para o MOM, a resolução do transporte dos momentos bivariados, aumentando significativamente o número de equações tratadas (FOX, 2006).

\subsubsection{Método Direto das Quadraturas dos Momentos (DQMOM)}

O QMOM foi uma evolução sobre o MOM, mas reteve em grande parte as limitações de seu antecessor. Necessitando de um método adequado para sistemas polidispersos, mas ainda com $\mathrm{o}$ intuito de manter a robustez e praticidade características dos métodos originados da proposta de Hulburt e Katz (1964), Fan; Marchisio e Fox (2004) propuseram a substituição direta dos termos aproximados por quadratura gaussiana na EBP, originando o Método Direto das Quadraturas dos Momentos.

Relembrando a contribuição de McGraw (1997) ao trabalho de Hulburt e Katz (1964):

$$
m_{k}=\int_{\Omega_{x}} x^{k} f(x, \boldsymbol{r}, t) \mathrm{d} x \cong \sum_{\alpha=1}^{N} \omega_{\alpha}(\boldsymbol{r}, t) x_{\alpha}{ }^{k}(\boldsymbol{r}, t), k=0, \ldots, 2 N-1
$$

Para que essa aproximação seja verdadeira, tem-se que:

$$
f(x, \boldsymbol{r}, t) \cong \sum_{\alpha=1}^{N} \omega_{\alpha}(\boldsymbol{r}, t) \delta\left(x-x_{\alpha}(\boldsymbol{r}, t)\right)
$$

Sendo $\delta\left(x-x_{\alpha}(\boldsymbol{r}, t)\right)$ a função delta de Dirac (Butkov, 1968). 
Substituindo essa relação diretamente na seguinte EBP (por simplificação, sem o termo de crescimento explicitado e sem o termo difusivo):

$$
\frac{\partial f(x, \boldsymbol{r}, t)}{\partial t}+\nabla_{\mathbf{r}} \cdot[\dot{\boldsymbol{R}}(\boldsymbol{r}, \boldsymbol{Y}, t) f(x, \boldsymbol{r}, t)]=S(x, \boldsymbol{Y}, t)
$$

Tem-se, após o agrupamento dos termos resultantes:

$$
\begin{gathered}
\sum_{\alpha=1}^{N} \delta\left(x-x_{\alpha}(\boldsymbol{r}, t)\right)\left[\frac{\partial \omega_{\alpha}(\boldsymbol{r}, t)}{\partial t}+\nabla_{\mathbf{r}} \cdot\left[\dot{\boldsymbol{R}}_{\alpha}(\boldsymbol{r}, \boldsymbol{Y}, t) \omega_{\alpha}(\boldsymbol{r}, t)\right]\right] \\
-\sum_{\alpha=1}^{N} \delta^{\prime}\left(x-x_{\alpha}(\boldsymbol{r}, t)\right)\left[\frac{\partial x_{\alpha}(\boldsymbol{r}, t)}{\partial t}+\dot{\boldsymbol{R}}_{\alpha}(\boldsymbol{r}, \boldsymbol{Y}, t)\right. \\
\left.\cdot \nabla_{\mathbf{r}} x_{\alpha}(\boldsymbol{r}, t)\right]=S(x, \boldsymbol{Y}, t)
\end{gathered}
$$

Sendo $\delta^{\prime}\left(x-x_{\alpha}(\boldsymbol{r}, t)\right)$ a derivada de primeira ordem da função delta de Dirac.

Em vez de acompanhar a evolução dos momentos da distribuição, o DQMOM aplica equações de transporte aos pesos e abscissas ponderadas da quadratura. Sendo a abscissa ponderada definida como produto da abscissa e seu peso correspondente:

$$
\varsigma_{\alpha}(\boldsymbol{r}, t) \equiv \omega_{\alpha}(\boldsymbol{r}, t) x_{\alpha}(\boldsymbol{r}, t)
$$

É possível substituir essa definição na equação anterior, obtendo-se: 


$$
\begin{aligned}
\sum_{\alpha=1}^{N} \delta\left(x-x_{\alpha}(\boldsymbol{r}, t)\right)\left[\frac{\partial \omega_{\alpha}(\boldsymbol{r}, t)}{\partial t}+\nabla_{\mathbf{r}} \cdot\left[\dot{\boldsymbol{R}}_{\alpha}(\boldsymbol{r}, \boldsymbol{Y}, t) \omega_{\alpha}(\boldsymbol{r}, t)\right]\right] \\
-\sum_{\alpha=1}^{N} \delta^{\prime}\left(x-x_{\alpha}(\boldsymbol{r}, t)\right)\left[\frac{\partial \zeta_{\alpha}(\boldsymbol{r}, t)}{\partial t}+\nabla_{\mathbf{r}}\right. \\
\cdot\left[\dot{\boldsymbol{R}}_{\alpha}(\boldsymbol{r}, \boldsymbol{Y}, t) \varsigma_{\alpha}(\boldsymbol{r}, t)\right] \\
-x_{\alpha}(\boldsymbol{r}, t)\left[\frac{\partial \omega_{\alpha}(\boldsymbol{r}, t)}{\partial t}+\nabla_{\mathbf{r}} \cdot\left[\dot{\boldsymbol{R}}_{\alpha}(\boldsymbol{r}, \boldsymbol{Y}, t) \omega_{\alpha}(\boldsymbol{r}, t)\right]\right] \\
=S(x, \boldsymbol{Y}, t)
\end{aligned}
$$

Desse resultado são obtidas as equações de transporte do método:

$$
\begin{aligned}
& \frac{\partial \omega_{\alpha}(\boldsymbol{r}, t)}{\partial t}+\nabla_{\mathbf{r}} \cdot\left[\dot{\boldsymbol{R}}_{\alpha}(\boldsymbol{r}, \boldsymbol{Y}, t) \omega_{\alpha}(\boldsymbol{r}, t)\right]=S_{\omega_{\alpha}}(\boldsymbol{r}, \boldsymbol{Y}, t) \\
& \frac{\partial \zeta_{\alpha}(\boldsymbol{r}, t)}{\partial t}+\nabla_{\mathbf{r}} \cdot\left[\dot{\boldsymbol{R}}_{\alpha}(\boldsymbol{r}, \boldsymbol{Y}, t) \zeta_{\alpha}(\boldsymbol{r}, t)\right]=S_{\zeta_{\alpha}}(\boldsymbol{r}, \boldsymbol{Y}, t)
\end{aligned}
$$

Introduzindo os termos fonte dos pesos e das abscissas ponderadas, $S_{\omega_{\alpha}}(\boldsymbol{r}, \boldsymbol{Y}, t)$ e $S_{\zeta \alpha}(\boldsymbol{r}, \boldsymbol{Y}, t)$ respectivamente. Dessa forma, é possível reescrever a EBP:

$$
\begin{array}{r}
\sum_{\alpha=1}^{N}\left[\delta\left(x-x_{\alpha}(\boldsymbol{r}, t)\right)-\delta^{\prime}\left(x-x_{\alpha}(\boldsymbol{r}, t)\right) x_{\alpha}(\boldsymbol{r}, t)\right] S_{\omega_{\alpha}}(\boldsymbol{r}, \boldsymbol{Y}, t) \\
-\sum_{\alpha=1}^{N} \delta^{\prime}\left(x-x_{\alpha}(\boldsymbol{r}, t)\right) S_{\zeta_{\alpha}}(\boldsymbol{r}, \boldsymbol{Y}, t)=S(x, \boldsymbol{Y}, t)
\end{array}
$$

O próximo passo é integrar essa equação de forma similar à formulação dos momentos da distribuição de partículas, tirando proveito das propriedades da função delta de Dirac. Mais especificamente:

$$
\begin{gathered}
\int_{\Omega_{x}} x^{k} \delta\left(x-x_{\alpha}(\boldsymbol{r}, t)\right) \mathrm{d} x=x_{\alpha}{ }^{k}(\boldsymbol{r}, t) \\
\int_{\Omega_{x}} x^{k} \delta^{\prime}\left(x-x_{\alpha}(\boldsymbol{r}, t)\right) \mathrm{d} x=-k x_{\alpha}{ }^{k-1}(\boldsymbol{r}, t)
\end{gathered}
$$


Resultando em:

$$
\begin{gathered}
(1-k) \sum_{\alpha=1}^{N} x_{\alpha}{ }^{k}(\boldsymbol{r}, t) S_{\omega_{\alpha}}(\boldsymbol{r}, \boldsymbol{Y}, t)+k \sum_{\alpha=1}^{N} x_{\alpha}{ }^{k-1}(\boldsymbol{r}, t) S_{\zeta \alpha}(\boldsymbol{r}, \boldsymbol{Y}, t) \\
=\bar{S}_{k}^{(N)}(\boldsymbol{Y}, t)
\end{gathered}
$$

Sendo $\bar{S}_{k}^{(N)}(\boldsymbol{Y}, t)$ o momento de ordem $k$ do termo fonte $S(x, \boldsymbol{Y}, t)$, descrito pela equação a seguir, para um caso de agregação e quebra sem crescimento de partículas:

$$
\begin{aligned}
\bar{S}_{k}^{(N)}(\boldsymbol{Y}, t)= & \int_{\Omega_{x}} x^{k} S(x, \boldsymbol{Y}, t) \mathrm{d} x \\
& =\bar{B}_{k}^{A}(\boldsymbol{Y}, t)+\bar{B}_{k}^{B}(\boldsymbol{Y}, t)-\bar{D}_{k}^{A}(\boldsymbol{Y}, t)-\bar{D}_{k}^{B}(\boldsymbol{Y}, t)
\end{aligned}
$$

E os momentos dos termos de agregação e quebra resultantes desse tratamento matemático, após as devidas mudanças dos domínios de integração como vistas no livro de Ramkrishna (2000):

$$
\begin{aligned}
& \bar{D}_{k}^{A}(\boldsymbol{Y}, t)=\int_{0}^{\infty} x^{k}\left[f(x, t) \int_{0}^{\infty} a\left(x^{\prime} ; x ; \boldsymbol{Y}, t\right) f\left(x^{\prime}, t\right) \mathrm{d} x^{\prime}\right] \mathrm{d} x \\
&=\sum_{\alpha=1}^{N} \sum_{\beta=1}^{N} \omega_{\alpha}(\boldsymbol{r}, t) \omega_{\beta}(\boldsymbol{r}, t) x_{\alpha}{ }^{k}(\boldsymbol{r}, t) a_{\beta \alpha}(\boldsymbol{Y}, t) \\
& \bar{B}_{k}^{A}(\boldsymbol{Y}, t)=\int_{0}^{\infty} x^{k}\left[\frac{1}{2} \int_{0}^{\infty} a\left(x^{\prime \prime} ; x^{\prime} ; \boldsymbol{Y}, t\right) f\left(x^{\prime \prime}, t\right) f\left(x^{\prime}, t\right) \mathrm{d} x^{\prime \prime}\right] \mathrm{d} x \\
&=\int_{0}^{\infty}\left[x^{\prime \prime}\right. \\
&\left.+x^{\prime}\right]^{k} \frac{1}{2} \int_{0}^{\infty} a\left(x^{\prime \prime} ; x^{\prime} ; \boldsymbol{Y}, t\right) f\left(x^{\prime \prime}, t\right) f\left(x^{\prime}, t\right) \mathrm{d} x^{\prime \prime} \mathrm{d} x^{\prime} \\
&=\frac{1}{2} \sum_{\alpha=1}^{N} \sum_{\beta=1}^{N} \omega_{\beta}(\boldsymbol{r}, t) \omega_{\alpha}(\boldsymbol{r}, t)\left[x_{\beta}(\boldsymbol{r}, t)\right. \\
&\left.+x_{\alpha}(\boldsymbol{r}, t)\right]^{k} a_{\beta \alpha}(\boldsymbol{Y}, t)
\end{aligned}
$$




$$
\begin{gathered}
\bar{D}_{k}^{B}(\boldsymbol{Y}, t)=\int_{0}^{\infty} x^{k} b(x, \boldsymbol{Y}, t) f(x, t) \mathrm{d} x=\sum_{\alpha=1}^{N} \omega_{\alpha}(\boldsymbol{r}, t) x_{\alpha}{ }^{k}(\boldsymbol{r}, t) b_{\alpha}(\boldsymbol{Y}, t) \\
\bar{B}_{k}^{B}(\boldsymbol{Y}, t)=\int_{0}^{\infty} x^{k}\left[2 \int_{x}^{\infty} b\left(x^{\prime}, \boldsymbol{Y}, t\right) P\left(x \mid x^{\prime}, \boldsymbol{Y}, t\right) f\left(x^{\prime}, t\right) \mathrm{d} x^{\prime}\right] \mathrm{d} x \\
=2 \int_{0}^{\infty} b\left(x^{\prime}, \boldsymbol{Y}, t\right) f\left(x^{\prime}, t\right)\left[\int_{0}^{x^{\prime}} x^{k} P\left(x \mid x^{\prime}, \boldsymbol{Y}, t\right) \mathrm{d} x\right] \mathrm{d} x^{\prime} \\
=2 \sum_{\alpha=1}^{N} \omega_{\alpha}(\boldsymbol{r}, t) b_{\alpha}(\boldsymbol{Y}, t) \bar{P}_{\alpha}^{k}(\boldsymbol{r}, t)
\end{gathered}
$$

Com:

$$
\bar{P}_{\alpha}^{k}(\boldsymbol{r}, t)=\int_{0}^{x_{\alpha}} x^{k} P\left(x \mid x_{\alpha}, \boldsymbol{Y}, t\right) \mathrm{d} x
$$

Esse sistema de equações algébricas permite calcular os termos fonte das equações de transporte dos pesos $\omega_{\alpha}$ e abscissas ponderadas $\varsigma_{\alpha}$, cuja resolução permite, por sua vez, o cálculo dos momentos da distribuição.

Essa abordagem representa uma grande evolução sobre o QMOM, contornando tanto limitações próprias do mesmo quanto herdadas do MOM. A mesma aproximação dos momentos por quadratura gaussiana é utilizada e, então, o fechamento dos termos fonte é mantido. A contribuição principal desse método é permitir a modelagem da diferença de velocidade entre partículas de tamanhos distintos. Apesar da EBP em que se baseia apresentar um campo de velocidades independente da coordenada interna, cada ponto $\alpha$ da quadratura pode ser interpretado como uma fase distinta com seu próprio campo de velocidades $\dot{\boldsymbol{R}}_{\alpha}(\boldsymbol{r}, \boldsymbol{Y}, t)$ (FAN; MARCHISIO; FOX, 2004). Outra característica notável é que, ao contrário de seus antecessores, seu equacionamento para casos multivariados é essencialmente o mesmo do caso monovariado e não gera um aumento grande do número de equações a serem trabalhadas (MARCHISIO; FOX, 2005). Além disso, a resolução desses casos torna-se muito mais simples ao se eliminar a necessidade da utilização do algoritmo PD para o cálculo dos pesos e abscissas (FOX, 2006), apesar do mesmo ainda ser útil, mas não imprescindível, para a inicialização desses valores em simulações CFD (SILVA; DAMIAN; LAGE, 2008). 
Apesar dos avanços obtidos, o sistema de equações 2.4.3.12 gera uma matriz mal condicionada que pode levar o método a divergir dependendo dos valores dos pesos e abscissas alcançados. Discussão maior sobre maneiras de contornar esse problema quando significante foi feita por Marchisio e Fox (2005) e Su et al. (2008).

\section{MATERIAIS E MÉTODOS}

\subsection{Reprodução do DQMOM}

O primeiro passo do trabalho foi obter uma implementação confiável do método, e um maior entendimento do seu funcionamento. Damian (2007) realizou um estudo da viabilidade do acoplamento do DQMOM ao simulador CFD comercial Ansys Cfx 11.0. Para fins de validação foi utilizada a solução analítica da EBP desenvolvida por McCoy e Madras (2003), válida para dimensões numéricas específicas dos termos de agregação e quebra de partículas e uma distribuição inicial pré-definida. Utilizando as mesmas condições iniciais em ambos os métodos foi mostrado que, apesar da incerteza inerente à aproximação dos momentos por quadratura gaussiana, os resultados obtidos são condizentes com o esperado.

Utilizando então o software Microsoft Excel 2007 para obter os momentos da distribuição analiticamente e o compilador Bloodshed Dev-C++ 5.0 para compilar a rotina $\mathrm{DQMOM}$ em linguagem $\mathrm{C}$ e, com esta, obter os momentos aproximados, foi possível realizar a validação do método com os resultados exibidos por Damian (2007).

Para a seguinte EBP, que descreve um problema de agregação e quebra sem escoamento:

$$
\begin{gathered}
\frac{\partial f(x, t)}{\partial t}=B^{A}(x, t)+B^{B}(x, t)-D^{A}(x, t)-D^{B}(x, t) \\
B^{A}(x, t)=\frac{1}{2} \int_{0}^{\infty} a f\left(x-x^{\prime}, t\right) f\left(x^{\prime}, t\right) \mathrm{d} x^{\prime \prime}
\end{gathered}
$$




$$
\begin{gathered}
B^{B}(x, t)=2 \int_{x}^{\infty} b x \frac{1}{x^{\prime}} f\left(x^{\prime}, t\right) \mathrm{d} x^{\prime} \\
D^{A}(x, t)=f(x, t) \int_{0}^{\infty} a f\left(x^{\prime}, t\right) \mathrm{d} x^{\prime} \\
D^{B}(x, Y, t)=b x f(x, t)
\end{gathered}
$$

Com $a$ e $b$ constantes $(b(x, t)=b x)$, a solução analítica correspondente à condição inicial:

$$
f(x, 0)=\frac{\left[m_{0}^{0}\right]^{2}}{m_{1}^{0}} e^{-\frac{m_{0}^{0}}{m_{1}^{0}} x}
$$

É dada por:

$$
\phi(\eta, \tau)=\Phi^{2}(\tau) e^{-\eta \Phi(\tau)}
$$

Sendo:

$$
\begin{gathered}
\Phi(\tau)=\Phi(\infty)\left[\frac{1+\Phi(\infty) \tanh \left(\Phi(\infty)^{\tau} / 2\right)}{\Phi(\infty)+\tanh \left(\Phi(\infty)^{\tau} / 2\right)}\right] \\
\Phi(\infty)=\frac{\sqrt{\frac{2 b m_{1}^{0}}{a}}}{m_{0}^{0}}
\end{gathered}
$$

E as grandezas adimensionalizadas:

$$
\begin{gathered}
\tau=m_{0}^{0} a t \\
\eta=x \frac{m_{0}^{0}}{m_{1}^{0}} \\
\phi(\eta, \tau)=f(x, t) \frac{m_{1}^{0}}{\left[m_{0}^{0}\right]^{2}}
\end{gathered}
$$




$$
\Phi(\tau)=\frac{m_{0}(t)}{m_{0}^{0}}
$$

Tomando a freqüência de agregação $a=1$ constante, tem-se, pela definição de $\Phi(\infty)$ :

$$
b=\frac{\Phi^{2}(\infty)}{2}
$$

O que faz com que $\Phi(\infty)$, a solução do balanço adimensionalizado em regime estacionário, seja o único parâmetro do problema.

Os momentos da distribuição podem ser então calculados por (DAMIAN, 2007):

$$
m_{k}(\tau)=\left[\frac{\Phi(\infty)+\tanh (\Phi(\infty) \tau / 2)}{\Phi(\infty)\left[1+\Phi(\infty) \tanh \left(\Phi(\infty)^{\tau} / 2\right)\right]}\right]^{k-1} k !, k \in \mathbb{N}
$$

E os momentos da distribuição inicial por:

$$
m_{k}^{0}=k !
$$

Para essa distribuição inicial, os pesos e abscissas obtidos pelo algoritmo PD de Gordon (1968) para $N=4$ pontos de quadratura (a mesma quantidade exibida por Damian (2007)) são os mostrados na tabela 3.1.1.

Tabela 3.1.1 - Pesos e abscissas iniciais para validação do DQMOM

\begin{tabular}{ccc}
\hline$\alpha$ & $\omega_{\alpha}$ & $\xi_{\alpha}$ \\
\hline 1 & $6,0315 \cdot 10^{-1}$ & $3,2255 \cdot 10^{-1}$ \\
2 & $3,5742 \cdot 10^{-1}$ & $1,7458 \cdot 10^{0}$ \\
3 & $3,8888 \cdot 10^{-2}$ & $4,5366 \cdot 10^{0}$ \\
4 & $5,3929 \cdot 10^{-4}$ & $9,3951 \cdot 10^{-1}$ \\
\hline
\end{tabular}


As equações de transporte dos pesos e abscissas ponderadas sem convecção se reduzem a:

$$
\begin{aligned}
& \frac{\partial \omega_{\alpha}(\boldsymbol{r}, t)}{\partial t}=S_{\omega_{\alpha}}(\boldsymbol{r}, \boldsymbol{Y}, t) \\
& \frac{\partial \zeta_{\alpha}(\boldsymbol{r}, t)}{\partial t}=S_{\zeta_{\alpha}}(\boldsymbol{r}, \boldsymbol{Y}, t)
\end{aligned}
$$

Que, se discretizadas no tempo por meio do esquema explícito, resultam em:

$$
\begin{gathered}
\omega_{\alpha}{ }^{t_{i}}-\omega_{\alpha}{ }^{t_{i-1}}=S_{\omega_{\alpha}}{ }^{t_{i-1}} \\
S_{\alpha}{ }^{t_{i}}-\zeta_{\alpha}{ }^{t_{i-1}}=S_{\zeta_{\alpha}}{ }^{t_{i-1}}, i \geq 1
\end{gathered}
$$

E o $k$-ésimo momento do termo fonte $\bar{S}_{k}^{(N)}(t)$ da equação de transporte da densidade numérica de partículas, necessário para o cálculo dos termos fontes das mesmas, se reduz a:

$$
\begin{gathered}
\bar{S}_{k}^{(N)}(t)=\bar{B}_{k}^{A}(t)+\bar{B}_{k}^{B}(t)-\bar{D}_{k}^{A}(t)-\bar{D}_{k}^{B}(t) \\
\bar{B}_{k}^{A}(t)=\frac{1}{2} \sum_{\alpha=1}^{N} \sum_{\beta=1}^{N} \omega_{\beta}(t) \omega_{\alpha}(t)\left[x_{\beta}(t)+x_{\alpha}(t)\right]^{k} \\
\bar{B}_{k}^{B}(t)=\frac{\Phi^{2}(\infty)}{k+1} \sum_{\alpha=1}^{N} \omega_{\alpha}(t) x_{\alpha}{ }^{k}(t) \\
\bar{D}_{k}^{A}(t)=\sum_{\alpha=1}^{N} \sum_{\beta=1}^{N} \omega_{\alpha}(t) \omega_{\beta}(t) x_{\alpha}{ }^{k}(t) \\
\bar{D}_{k}^{B}(t)=\frac{\Phi^{2}(\infty)}{2} \sum_{\alpha=1}^{N} \omega_{\alpha}(\boldsymbol{r}, t) x_{\alpha}{ }^{k}(\boldsymbol{r}, t)
\end{gathered}
$$

Três casos foram estudados: agregação dominante $(\Phi(\infty)=0,1)$, quebra dominante $(\Phi(\infty)=10)$, e distribuição constante $(\Phi(\infty)=1)$, com mil passos temporais de 0,04, 0,001 e 0,04 segundos cada, respectivamente. Os resultados obtidos pelo DQMOM foram: 


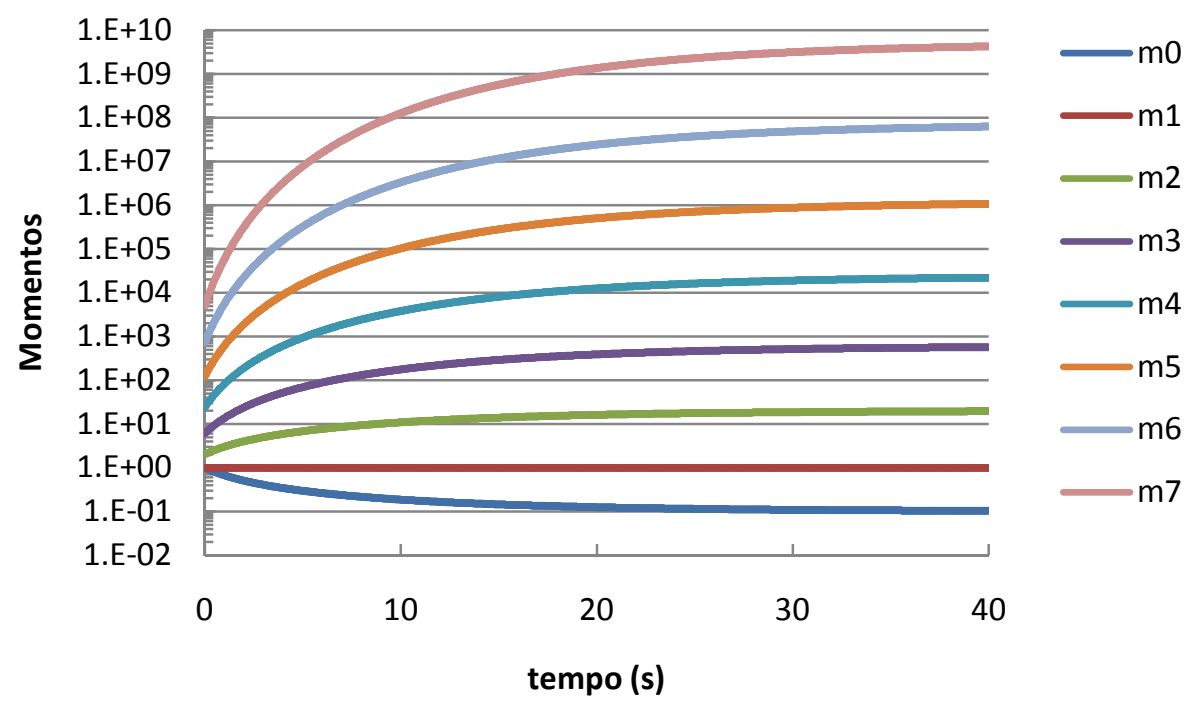

Figura 3.1.1 - Solução do DQMOM para a EBP de McCoy e Madras (2003) com agregação dominante $(\Phi(\infty)=0,1)$

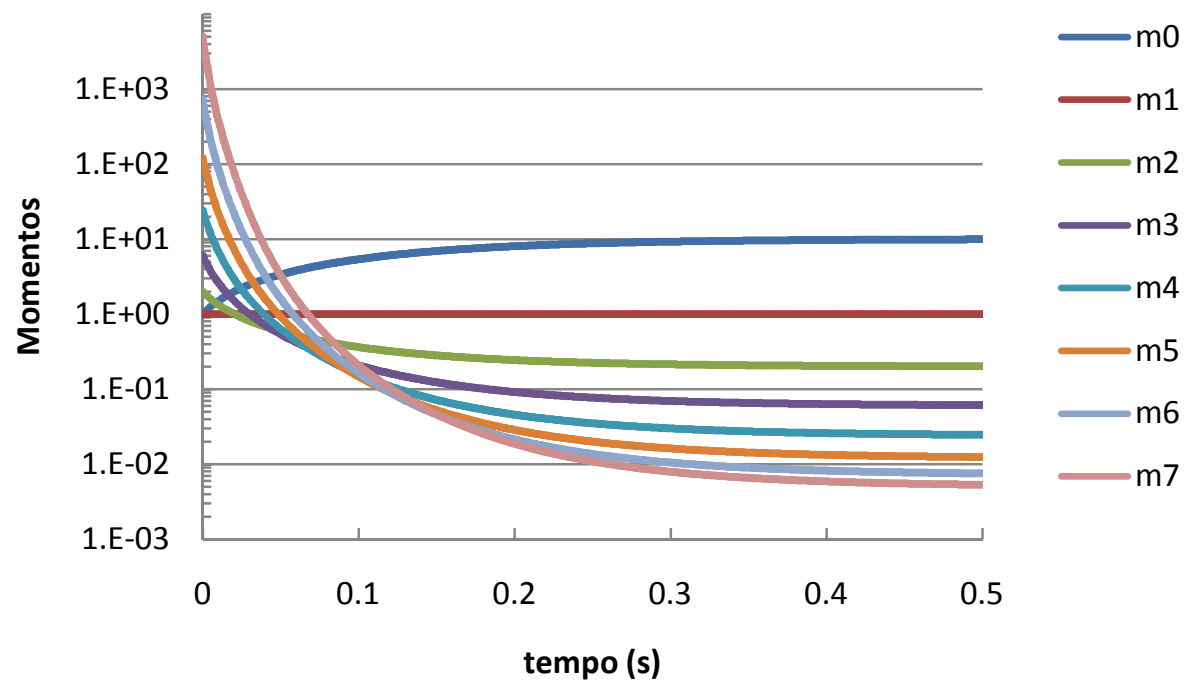

Figura 3.1.2 - Solução do DQMOM para a EBP de McCoy e Madras (2003) com quebra dominante $(\Phi(\infty)=10)$ 


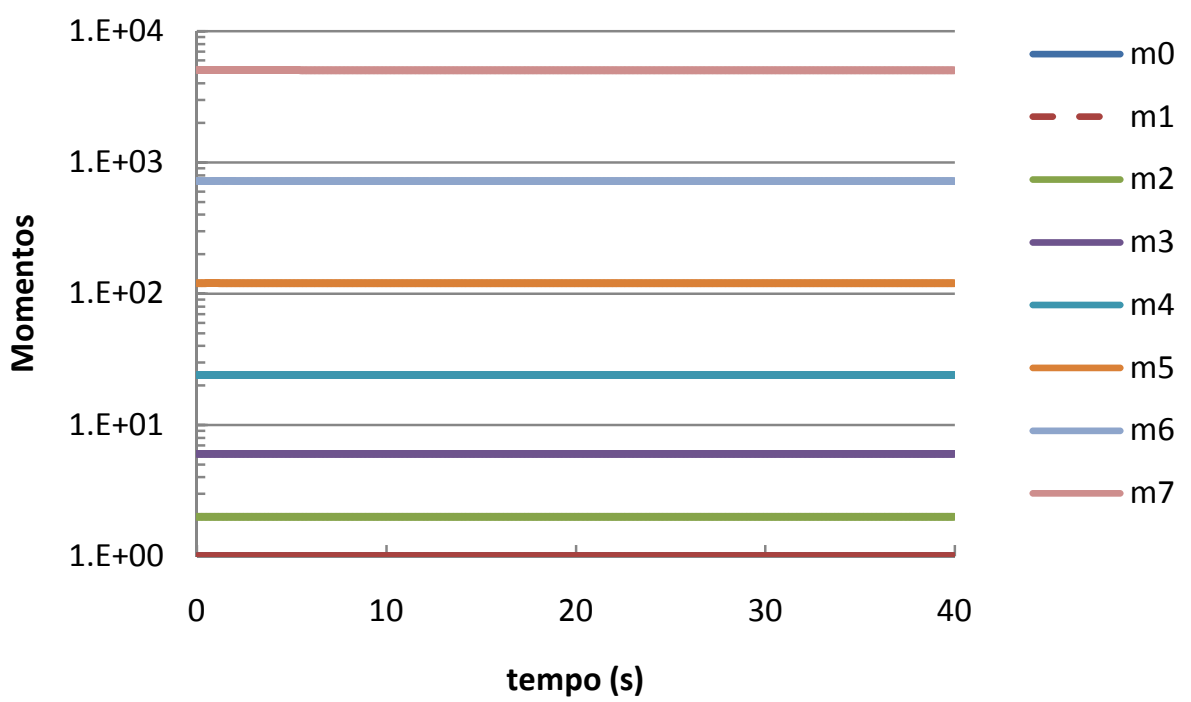

Figura 3.1.3 - Solução do DQMOM para a EBP de McCoy e Madras (2003) com distribuição constante $(\Phi(\infty)=1)$

Os três resultados permitem concluir que a implementação do método foi, ao menos qualitativamente, correta. No caso com agregação dominante verifica-se um decréscimo do número de partículas do sistema $\left(m_{0}\right)$, enquanto a coordenada interna da distribuição (massa ou volume totais do sistema, nesse caso) mantém-se constante, logo que $m_{1}$ não varia. $O$ mesmo é observado para o caso de quebra dominante, com a diferença de que o número total de partículas do sistema aumenta. $O$ caso das frequências de agregação e quebra equilibradas exibe uma distribuição constante com o tempo, como se pode ver pela conservação de todos os momentos monitorados.

Faz-se necessário também observar os erros relativos à solução analítica: 


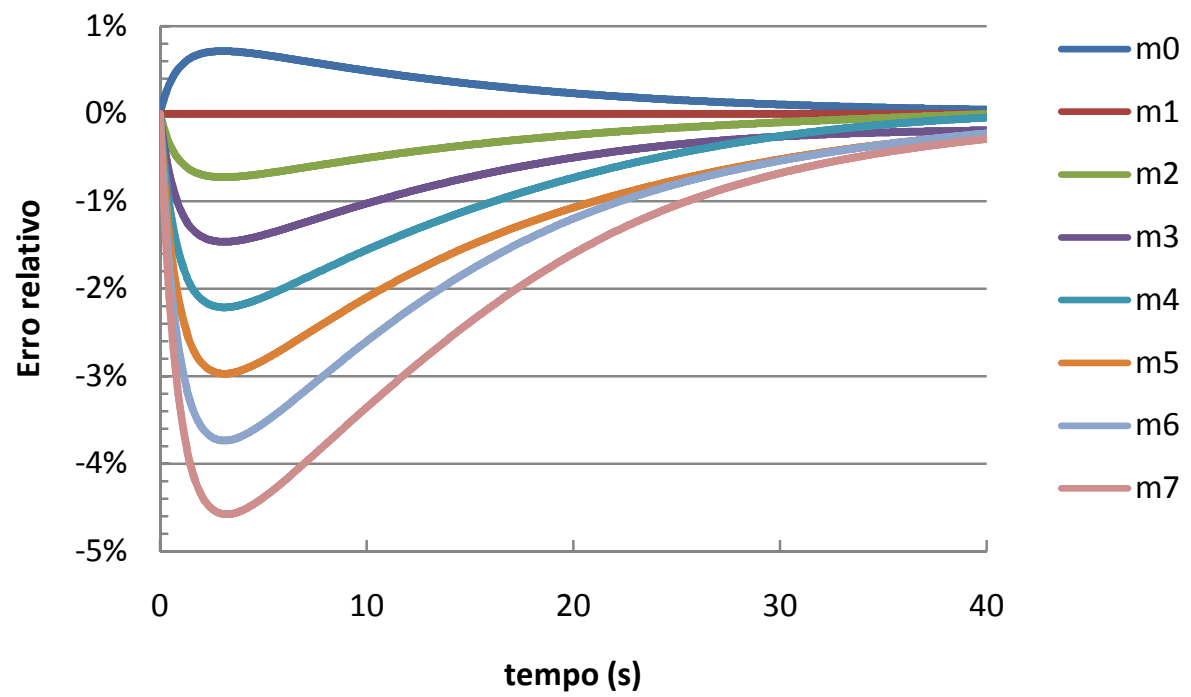

Figura 3.1.4 - Erro do DQMOM em relação à solução analítica da EBP de McCoy e Madras (2003) com agregação dominante $(\Phi(\infty)=0,1)$

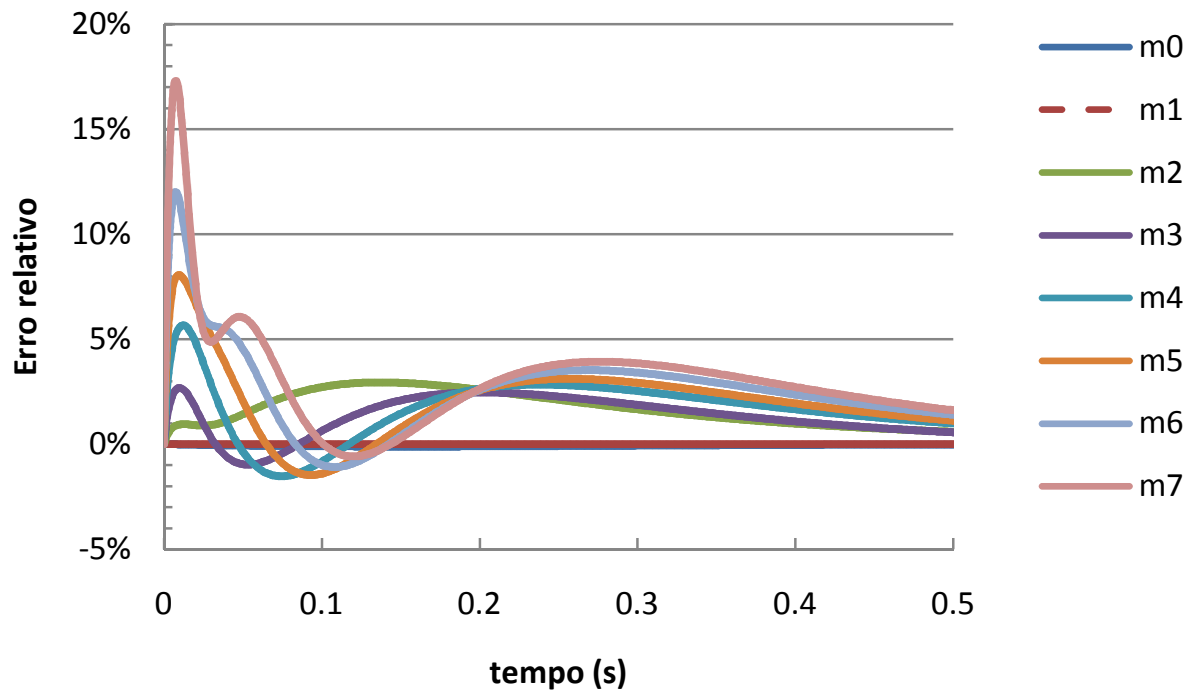

Figura 3.1.5 - Erro do DQMOM em relação à solução analítica da EBP de McCoy e Madras (2003) com quebra dominante $(\Phi(\infty)=10)$ 


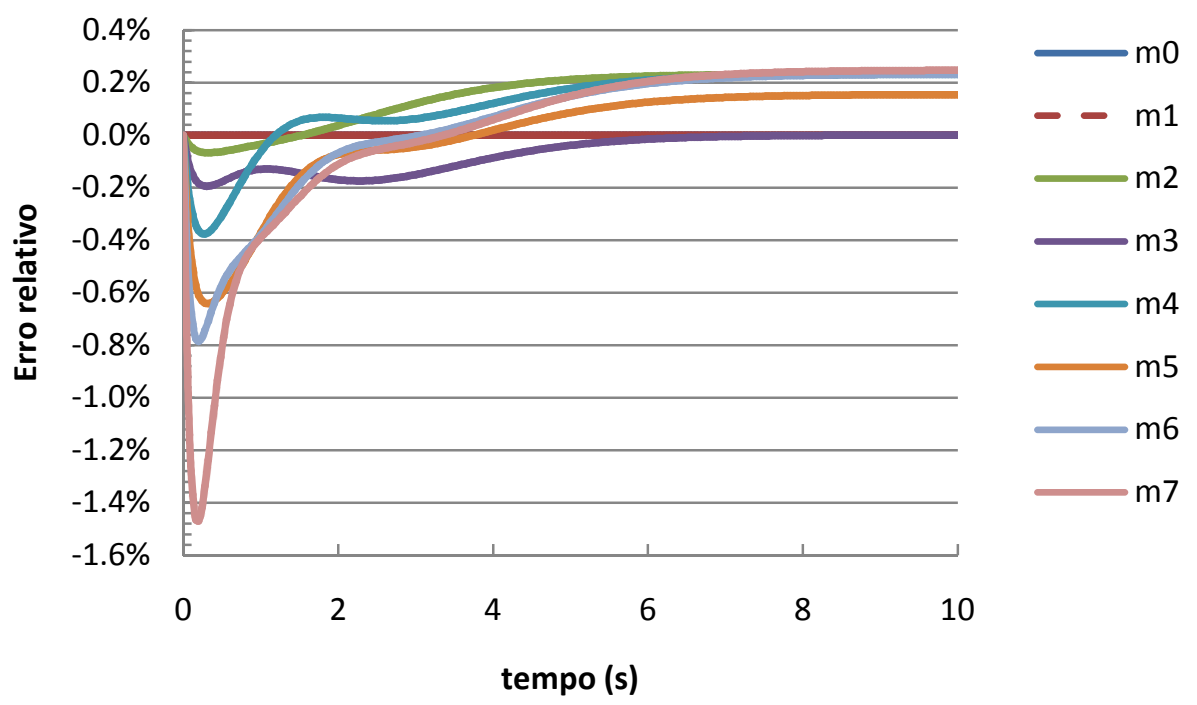

Figura 3.1.6 - Erro do DQMOM em relação à solução analítica da EBP de McCoy e Madras (2003) com distribuição constante $(\Phi(\infty)=1)$

Observa-se que, para todos os casos, os erros das soluções pelo método numérico são aceitáveis, apesar de serem bastante expressivos inicialmente, principalmente para o caso de quebra dominante, devido às maiores variações dos valores dos momentos nos instantes iniciais. Comparando esses resultados aos obtidos por Damian (2007) pode-se isolar o erro da aproximação por quadratura daquele referente à integração temporal, praticamente inexistente no trabalho anterior. Nota-se que o erro da integração temporal explícita é significativo, inviabilizando esse método simplificado de resolver o sistema de equações diferenciais do DQMOM. Apesar disso, os erros pequenos aos quais os momentos calculados tendem mostram que o método numérico conseguiu reproduzir o resultado analítico, comprovando finalmente sua implementação correta.

\subsection{Influência do Número de Pontos de Quadratura na Precisão do DQMOM}

Comprovada a implementação correta do método, faz-se necessária a determinação de seus parâmetros de forma que este corresponda acuradamente ao 
fenômeno estudado. Por sua simplicidade, o DQMOM exige apenas a especificação do número de pontos da aproximação por quadratura gaussiana dos momentos.

Em sua introdução do QMOM, McGraw (1997) adota em seus exemplos $N=3$ pontos de quadratura para representar as suas distribuições, aparentemente de forma arbitrária. Trabalhos subsequentes como os de Barret e Webb (1998), Marchisio; Virgil e Fox (2003a; 2003c), Marchisio et al. (2003b), Wan e Ring (2006) apóiam-se nesse precedente para justificar a mesma escolha. Marchisio e Fox (2005) provaram a equivalência dos resultados obtidos pelos métodos QMOM e DQMOM para casos monovariáveis, o que influenciou as escolhas feitas por Fan; Marchisio e Fox (2004) e Silva; Damian e Lage (2008). A falta de informação na literatura faz importante um levantamento do quanto a precisão do método é influenciada por este, seu único parâmetro.

Para isolar o erro da aproximação por quadratura, foi realizada outra implementação do DQMOM baseada na solução analítica de McCoy e Madras (2003), mas dessa vez as equações diferenciais foram resolvidas por meio do solver ODE45 do software Matlab R2009a, que se baseia num algoritmo Runge-Kutta explícito. A mesma verificação da implementação realizada para a solução por discretização explícita foi feita para validar os resultados obtidos.

Para o caso de agregação dominante, os erros do método para $N=2,34$ e 5 pontos de quadratura são, mostrados nas figuras 3.2.1 a 3.2.4. 


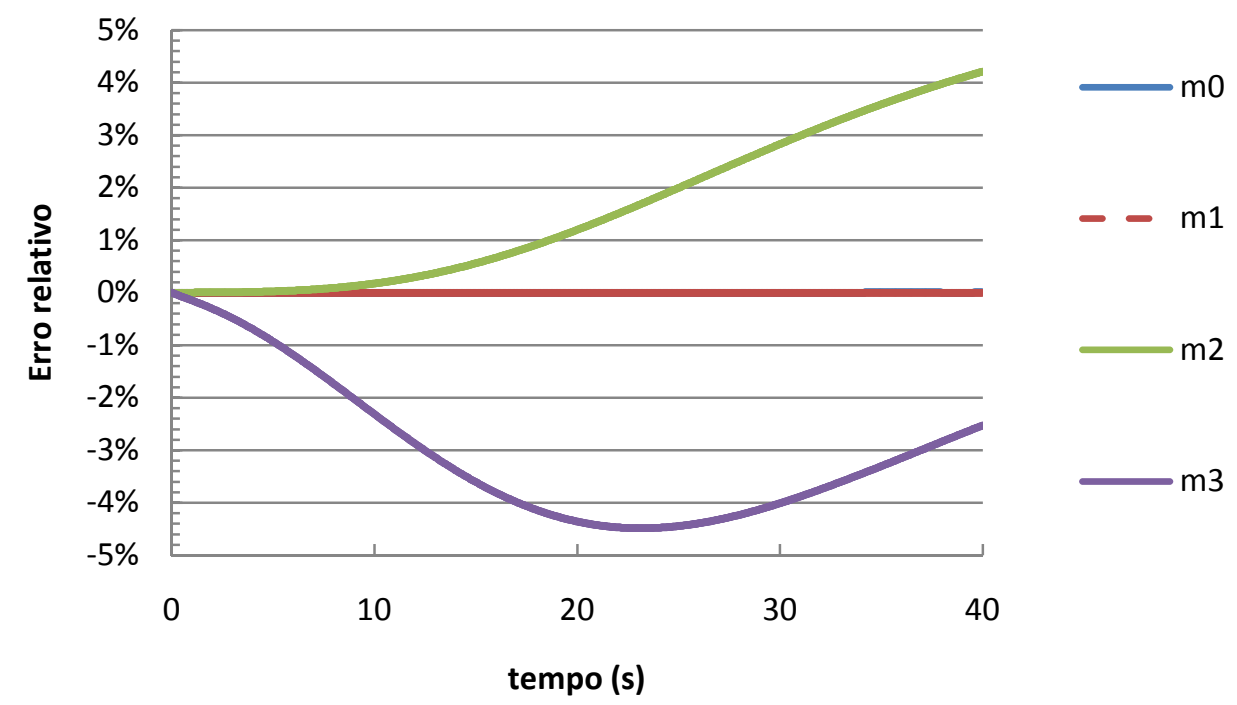

Figura 3.2.1 - Erro do DQMOM em relação à solução analítica da EBP de McCoy e Madras (2003) com agregação dominante $(\Phi(\infty)=0,1)$ e $N=2$ pontos de quadratura

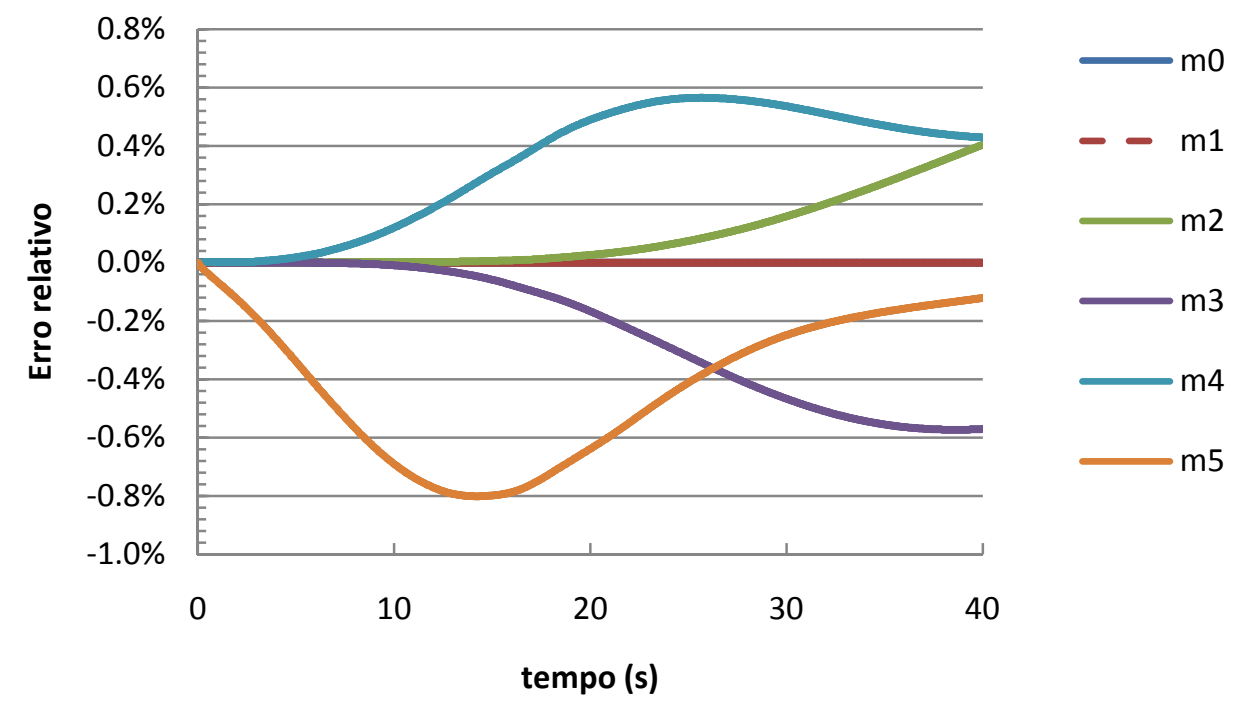

Figura 3.2.2 - Erro do DQMOM em relação à solução analítica da EBP de McCoy e Madras (2003) com agregação dominante $(\Phi(\infty)=0,1)$ e $N=3$ pontos de quadratura 


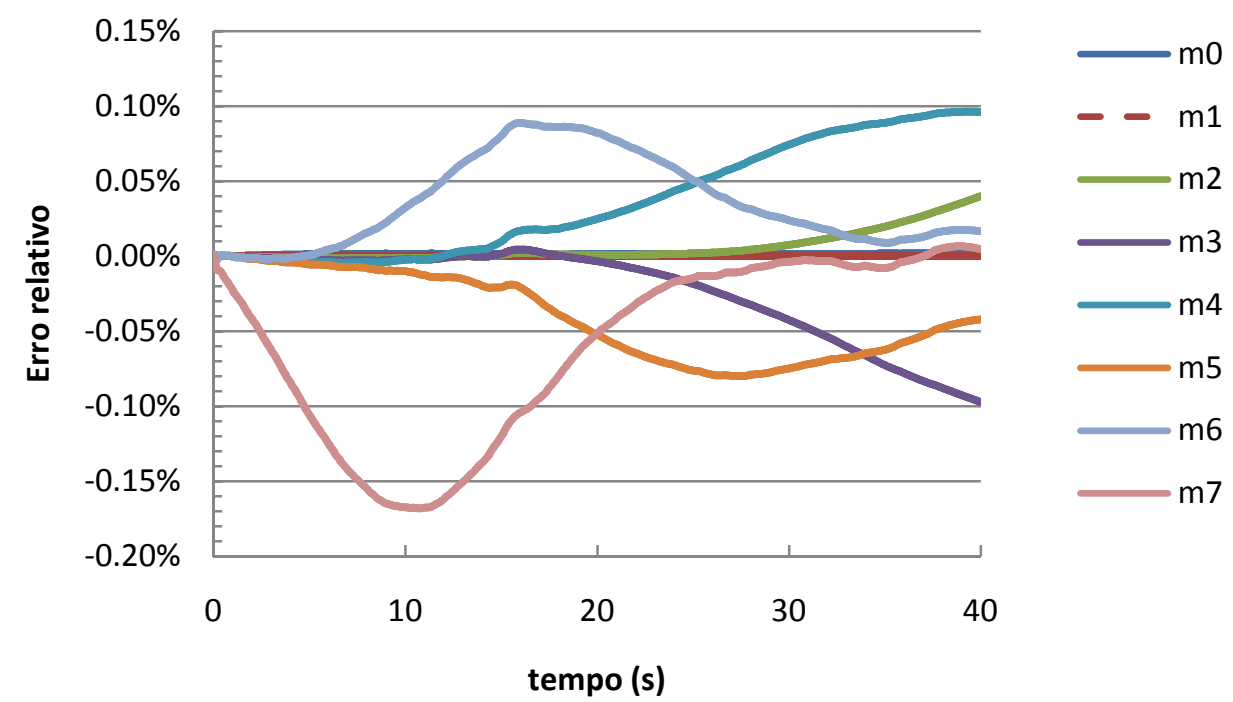

Figura 3.2.3 - Erro do DQMOM em relação à solução analítica da EBP de McCoy e Madras (2003) com agregação dominante $(\Phi(\infty)=0,1)$ e $N=4$ pontos de quadratura

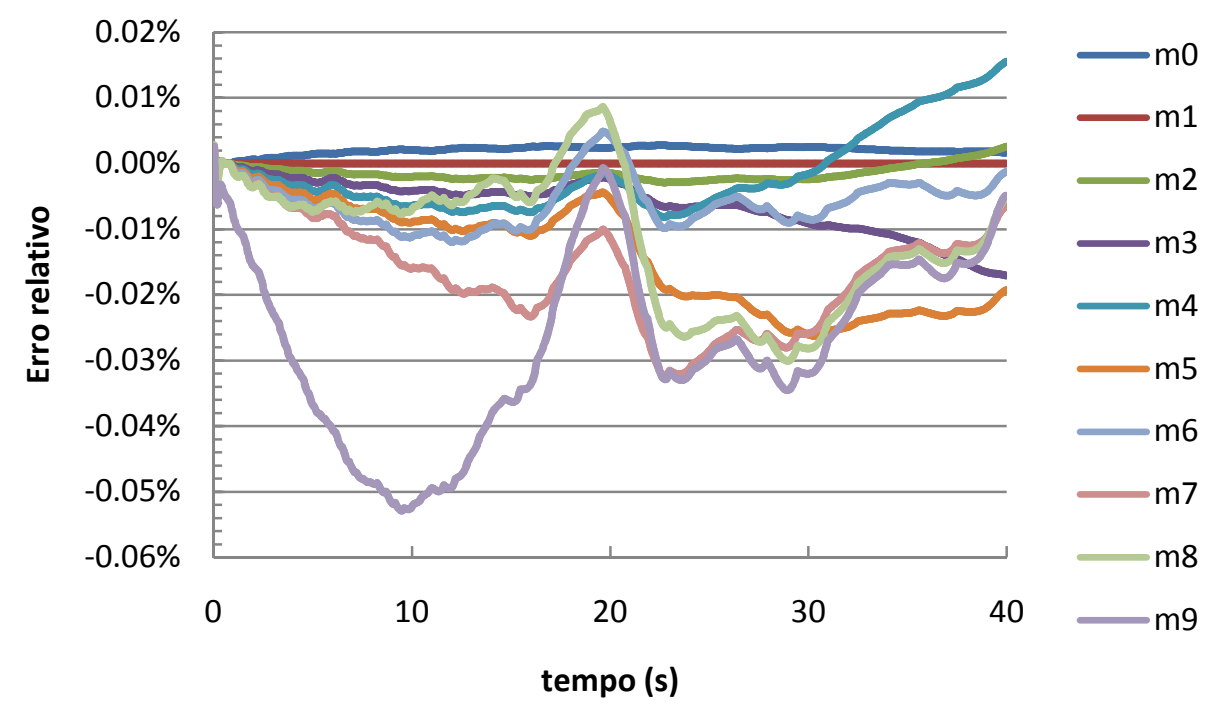

Figura 3.2.4 - Erro do DQMOM em relação à solução analítica da EBP de McCoy e Madras (2003) com agregação dominante $(\Phi(\infty)=0,1)$ e $N=5$ pontos de quadratura

É claro, nas figuras, o aumento da precisão do modelo com o aumento do número de pontos de quadratura. No entanto, aumenta também o esforço computacional requerido, apesar de este não ser grande. Como os erros para agregação dominante não se estabilizam com o tempo (ao contrário dos demais 
casos) e esse é o fenômeno estudado neste trabalho, o parâmetro $N=4$ foi adotado, apesar de a precisão obtida com $N=3$ ser suficientemente grande.

\section{RESULTADOS E DISCUSSÃO}

\subsection{Aplicação do Balanço Populacional}

Bresciani (2009) discutiu a dessalgação e propôs um modelo determinístico para a agregação de duas gotas de água emulsionadas no interior de uma dessalgadora, de acordo com o processo descrito previamente, dada uma distribuição inicial conhecida. Baseado em sua proposta foi desenvolvido um algoritmo que representa o processo lagrangeanamente por autômatos celulares, que é basicamente um método de discretização utilizado para viabilizar a computação dos balanços de forças entre as inúmeras gotas emulsionadas. No entanto, como a coleta de dados em etapas intermediárias da dessalgação não foi possível, somente os dados de entrada e saída do equipamento se fizeram disponíveis. Ou seja, a dinâmica de seu modelo proposto não pôde ser validada.

Apesar da evidente necessidade de estudos mais aprofundados do comportamento de emulsões água em óleo sob o efeito de corrente elétrica alternada, o modelo proposto foi considerado suficiente para dar prosseguimento a este trabalho. Os focos principais deste estudo são o balanço populacional de partículas em um sistema bifásico polidisperso, seu equacionamento e os métodos numéricos necessários para sua resolução eficiente.

\subsubsection{Modelo de Agregação}

O fechamento matemático do equacionamento do balanço populacional requer conhecimento da natureza dos fenômenos de agregação e quebra ocorrendo na fase dispersa. Na dessalgadora de alta velocidade existe a possibilidade de haver quebra de gotas por fenômenos diversos, principalmente quando o campo elétrico 
ao qual a emulsão é submetida é muito elevado, mas esse fenômeno é considerado desprezível nas condições aqui estudadas. Assim sendo, para a determinação do termo fonte da EBP foi necessário traduzir os dados coletados do algoritmo de autômatos celulares em uma função frequência de agregação (seção 2.3.1.).

Os resultados desse algoritmo consistem na distribuição de tamanhos de gotas ao longo do tempo, ou seja, nos $t=20$ segundos que um volume lagrangeano da emulsão demora para percorrer a distância de $r=1$ metro do dispersor até o fim do módulo de dessalgação, a velocidade constante (BRESCIANI, 2009), conforme mostrado na figura 4.1.1.

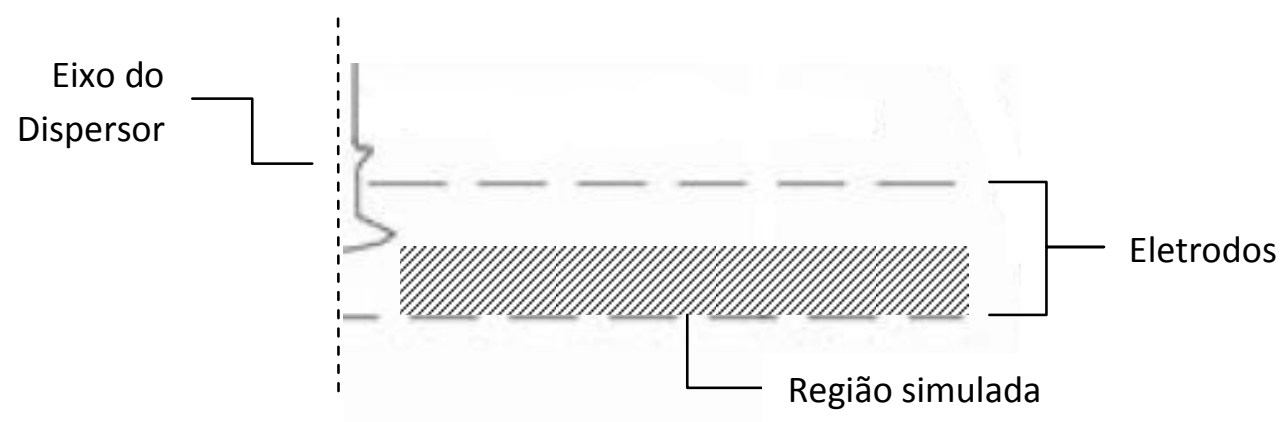

Figura 4.1.1 - Região do módulo de dessalgação considerada por Bresciani (2009), angularmente simétrica em torno do eixo do dispersor

Desses dados foi construída uma tabela de quantidade de gotas por faixa de tamanho por intervalo de tempo. Desconsiderando, assim como no trabalho original, a deformação das gotas pela ação do campo elétrico e de outros efeitos de polarização, é possível transformar as faixas de tamanho originais em faixas de volume de gotas esféricas, proporcional à sua massa, já que a massa específica da água é suposta constante. Obtém-se, então, a variação dos momentos da distribuição com o tempo, os mesmos resultados obtidos por qualquer método derivado do MOM.

Como parâmetros do algoritmo foram impostas células de 400 milímetros de largura por 400 milímetros de profundidade (e 225 milímetros de altura, pela região simulada), temperatura constante $T=120^{\circ} \mathrm{C}$, teor de água inicial de $7,0 \%$ em volume, campo elétrico alternado constante de $E=1,0 \mathrm{kV} / \mathrm{cm}$ e emulsão média, pela classificação de Bhardwaj e Hartland (1994). O volume e a quantidade de gotas foram adimensionalizados de acordo com: 


$$
\begin{gathered}
v^{a d m}=\frac{v}{\min (v)}=i^{3}, i=1,2, \ldots, 80 \\
n^{a d m}=\frac{n}{m_{0}^{0}}
\end{gathered}
$$

Sendo:

$$
m_{k}^{a d m}=\sum_{\alpha=1}^{\max (v)} n_{\alpha}^{a d m}\left[v_{\alpha}^{a d m}\right]^{k}
$$

Os momentos obtidos exibem o comportamento mostrado na figura 4.1.2.

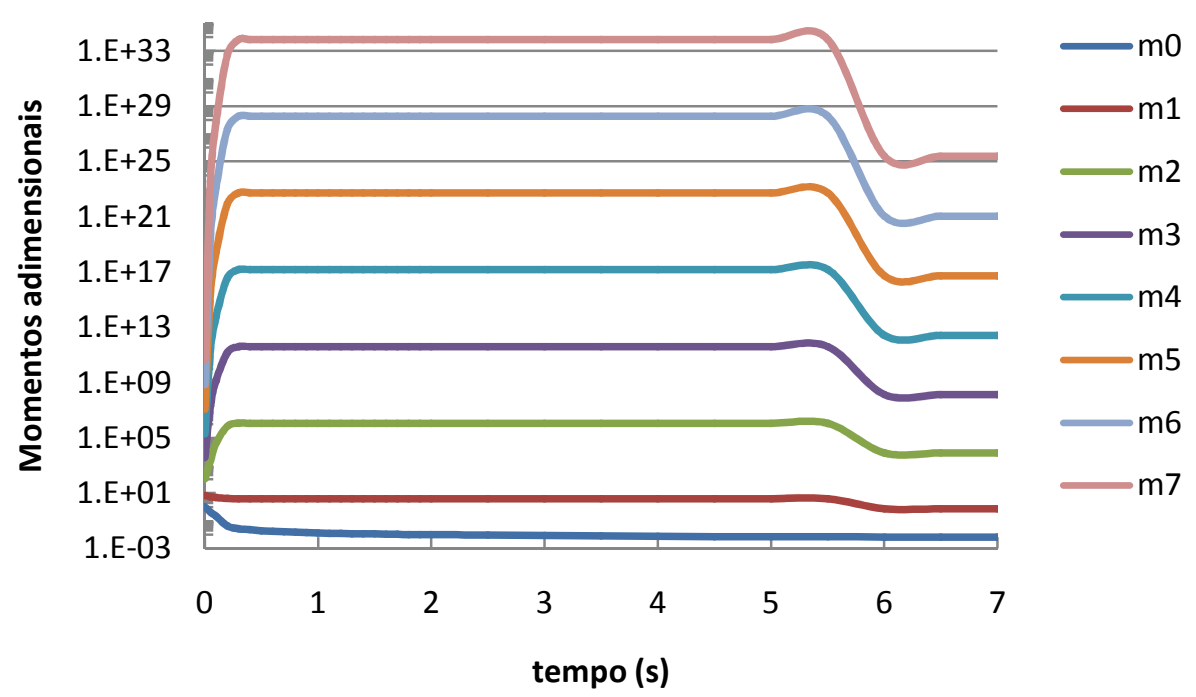

Figura 4.1.2 - Comportamento dos momentos obtidos pelo algoritmo de Bresciani (2009) para células de $400 \mathrm{~mm} \times 400 \mathrm{~mm}, T=120^{\circ} \mathrm{C}, 7,0 \%$ de água, $E=1,0 \mathrm{kV} /$ $\mathrm{cm}$, emulsão média e grandezas adimensionalizadas

Os resultados mostram uma queda abrupta dos momentos de ordem superior a $m_{0}$ devido à saída de uma grande quantidade de água do sistema entre os instantes $t=5,5 \mathrm{~s}$ e $t=6 \mathrm{~s}$. Para contornar essa característica, os valores dos momentos $m_{2}, m_{3}, \ldots, m_{7}$ posteriores a $t=5,5 \mathrm{~s}$, que já exibiam comportamento estacionário, foram mantidos constantes e os de $m_{1}$ (a massa total do sistema), foram extrapolados a partir de seu valor em $t=0 \mathrm{~s}$. Com essas alterações, os valores do algoritmo iniciais, finais e do instante $t=0,4 \mathrm{~s}$ (quando o comportamento 
do sistema muda) utilizados para a validação do kernel de agregação são os mostrados na tabela 4.1.1:

Tabela 4.1.1 - Momentos iniciais e finais obtidos pelo algoritmo de Bresciani (2009) para células de $400 \times 400, T=120{ }^{\circ} \mathrm{C}, 7,0 \%$ de água, $E=1,0 \mathrm{kV} / \mathrm{cm}$, emulsão média e grandezas adimensionalizadas

\begin{tabular}{cccc}
\hline$k$ & $m_{k}^{t=0 s}$ & $m_{k}^{t=0,4 s}$ & $m_{k}^{t=20 s}$ \\
\hline 0 & $1,0000 \cdot 10^{0}$ & $2,1532 \cdot 10^{-2}$ & $5,2323 \cdot 10^{-3}$ \\
1 & $6,6602 \cdot 10^{0}$ & $6,6602 \cdot 10^{0}$ & $6,6602 \cdot 10^{0}$ \\
2 & $1,0699 \cdot 10^{2}$ & $1,1067 \cdot 10^{6}$ & $1,1083 \cdot 10^{6}$ \\
3 & $3,7014 \cdot 10^{3}$ & $3,9403 \cdot 10^{11}$ & $3,9407 \cdot 10^{11}$ \\
4 & $1,9139 \cdot 10^{5}$ & $1,4099 \cdot 10^{17}$ & $1,4099 \cdot 10^{17}$ \\
5 & $1,1332 \cdot 10^{7}$ & $5,0464 \cdot 10^{22}$ & $5,0464 \cdot 10^{22}$ \\
6 & $7,0293 \cdot 10^{8}$ & $1,8061 \cdot 10^{28}$ & $1,8061 \cdot 10^{28}$ \\
7 & $4,4406 \cdot 10^{10}$ & $6,4644 \cdot 10^{33}$ & $6,4644 \cdot 10^{33}$ \\
\hline
\end{tabular}

Para encontrar a função frequência de agregação que melhor reproduzisse o comportamento dessa distribuição foi necessário realizar vários testes e comparar os erros relativos obtidos. Uma característica do DQMOM, identificada por Su et al. (2008), é a sensibilidade do condicionamento de seu sistema linear à magnitude relativa dos pesos e abscissas. Valores muito próximos podem fazer o método divergir, gerando abscissas e pesos negativos. Dessa forma, para esse exercício exploratório, foi realizada uma implementação do QMOM, método este que, como já discutido, é equivalente ao DQMOM para casos monovariáveis. Seu equacionamento para um sistema sem convexão ou difusão cujas partículas sofrem apenas o fenômeno de agregação é o seguinte:

$$
\frac{\partial m_{k}(\boldsymbol{r}, t)}{\partial t}=\bar{S}_{k}^{(4)}(\boldsymbol{r}, \boldsymbol{Y}, t), k=1,2, \ldots, 8
$$

Sendo o termo fonte o mesmo apresentado para o DQMOM:

$$
\bar{S}_{k}^{(4)}(t)=\bar{B}_{k}^{A}(t)-\bar{D}_{k}^{A}(t)
$$




$$
\begin{gathered}
\bar{B}_{k}^{A}(\boldsymbol{Y}, t)=\frac{1}{2} \sum_{\alpha=1}^{4} \sum_{\beta=1}^{4} \omega_{\beta}(\boldsymbol{r}, t) \omega_{\alpha}(\boldsymbol{r}, t)\left[x_{\beta}(\boldsymbol{r}, t)+x_{\alpha}(\boldsymbol{r}, t)\right]^{k} a_{\beta \alpha}(\boldsymbol{Y}, t) \\
\bar{D}_{k}^{A}(\boldsymbol{Y}, t)=\sum_{\alpha=1}^{4} \sum_{\beta=1}^{4} \omega_{\alpha}(\boldsymbol{r}, t) \omega_{\beta}(\boldsymbol{r}, t) x_{\alpha}{ }^{k}(\boldsymbol{r}, t) a_{\beta \alpha}(\boldsymbol{Y}, t)
\end{gathered}
$$

Os pesos e abscissas foram calculados a cada instante de tempo por meio do algoritmo PD de Gordon (1968), a partir do resultado do sistema de equações diferenciais obtido por meio do algoritmo Runge-Kutta explícito ODE45 do software Matlab R2009a.

Foi verificado que, para a dada formulação do QMOM, qualquer função frequência de agregação corretamente simétrica promove a conservação do momento de primeira ordem naturalmente (Apêndice A). Quando a variável interna escolhida é a massa do sistema (ou, como no caso aqui estudado, o volume dada uma massa específica constante), há conservação dessa propriedade automaticamente.

Para um modelo determinístico de deslocamento de partículas em um sistema disperso, conhecida a velocidade relativa entre um par de partículas qualquer, é normalmente possível a derivação de um modelo teórico de agregação (RAMKRISHNA, 2000). Apesar de Bresciani (2009) apresentar uma aproximação para essa grandeza, a influência que a distância entre as partículas de um dado par tem devido ao campo elétrico complica significativamente esse tratamento matemático. Uma maneira mais simples de se obter um modelo para esse fenômeno é a utilização de um kernel conhecido com os parâmetros adaptados de forma a se adequarem aos dados experimentais disponíveis (ADETAYO; ENNIS, 1997).

Há grande quantidade de modelos empíricos e teóricos na literatura, aplicados aos mais diversos casos de agregação e coalescência de diferentes tipos de partículas e gotas. Alguns modelos simples genéricos foram selecionados para que seu comportamento pudesse ser comparado ao do sistema estudado (Tabela 4.1.2). Diferentes valores das constantes existentes em cada formulação foram testados, objetivando sempre minimizar o erro relativo ao valor de $m_{0}^{t=20 s}$ mostrado na Tabela 4.1.1 ( $m_{1}$ é naturalmente conservado, como já discutido, fazendo com que seu erro seja inexistente). 
Tabela 4.1.2 - Funções frequência de agregação testadas

\begin{tabular}{ccc}
\hline Denominação & $a_{\beta \alpha}$ & Referência \\
\hline $\begin{array}{c}\text { Sonstante } \\
\text { Soma }\end{array}$ & $a\left[x_{\alpha}+x_{\beta}\right]$ & Hounslow e Ni (2004) \\
$\begin{array}{c}\text { Sisalhamento de } \\
\text { Produchowsky }\end{array}$ & $a\left[x_{\alpha}{ }^{1 / 3}+x_{\beta}{ }^{1 / 3}\right]^{3}$ & Hounslow e Ni (2004) \\
Kapur & $a x_{\alpha}{ }^{b} x_{\beta}{ }^{b}$ & Hounslow e Ni (2004) \\
Sastry & $a \frac{\left[x_{\alpha}+x_{\beta}\right]^{b}}{\left[x_{\alpha} x_{\beta}\right]^{c}}$ & Kapur (1972) \\
& $a \frac{\left[x_{\alpha}{ }^{2} / 3+x_{\beta}{ }^{2 / 3}\right]}{\left[\frac{1}{x_{\alpha}}+\frac{1}{x_{\beta}}\right]}$ & Liu e Litster (2002) \\
Adetayo e Ennis & $a \frac{\left[x_{\alpha} x_{\beta}\right]^{b}}{\left[x_{\alpha}+x_{\beta}\right]^{c}}$ & Adetayo e Ennis (1997) \\
\hline
\end{tabular}

A adoção do kernel Constante faz com que a taxa de agregação seja proporcional à quantidade de partículas no sistema. O fenômeno é mais expressivo inicialmente e tende suavemente ao estado estacionário. É o mesmo tipo de comportamento observado na Figura 4.1.2 (resultado do algoritmo de autômatos celulares), mas mais suave, como se pode ver pela Figura 4.1.3. 


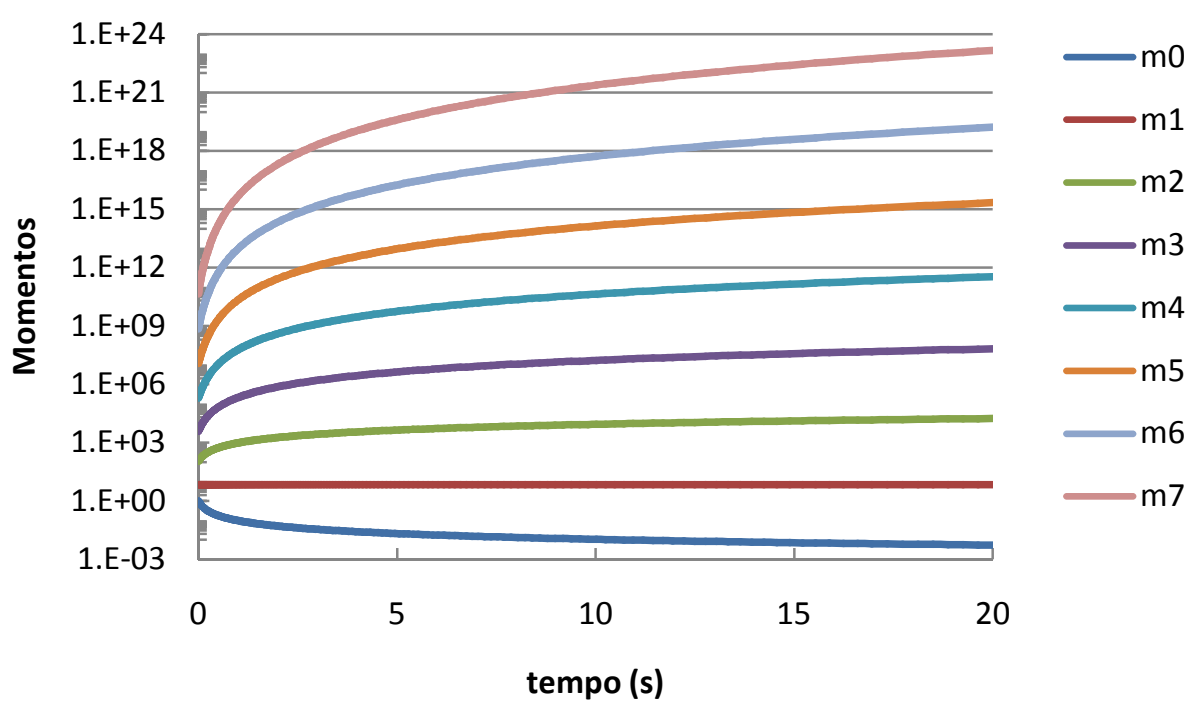

Figura 4.1.3 - Solução do QMOM proposto para um sistema sem escoamento ou difusão com agregação pura e Kernel Constante com $a=19$

Os kernéis Soma, Cisalhamento de Smoluchowsky e Produto dependem positivamente do valor da variável interna das partículas de cada par considerado. Assim sendo, com o progresso da simulação, há um aumento da intensidade de agregação observada, logo que as partículas tendem a concentrar valores maiores da variável interna, como mostram as Figuras 4.1.4 a 4.1.6. No caso específico do kernel Produto um decréscimo de $b$ faz com que seu comportamento se aproxime àquele do kernel Constante, enquanto o inverso intensifica a influência da magnitude da variável interna de cada partícula. 


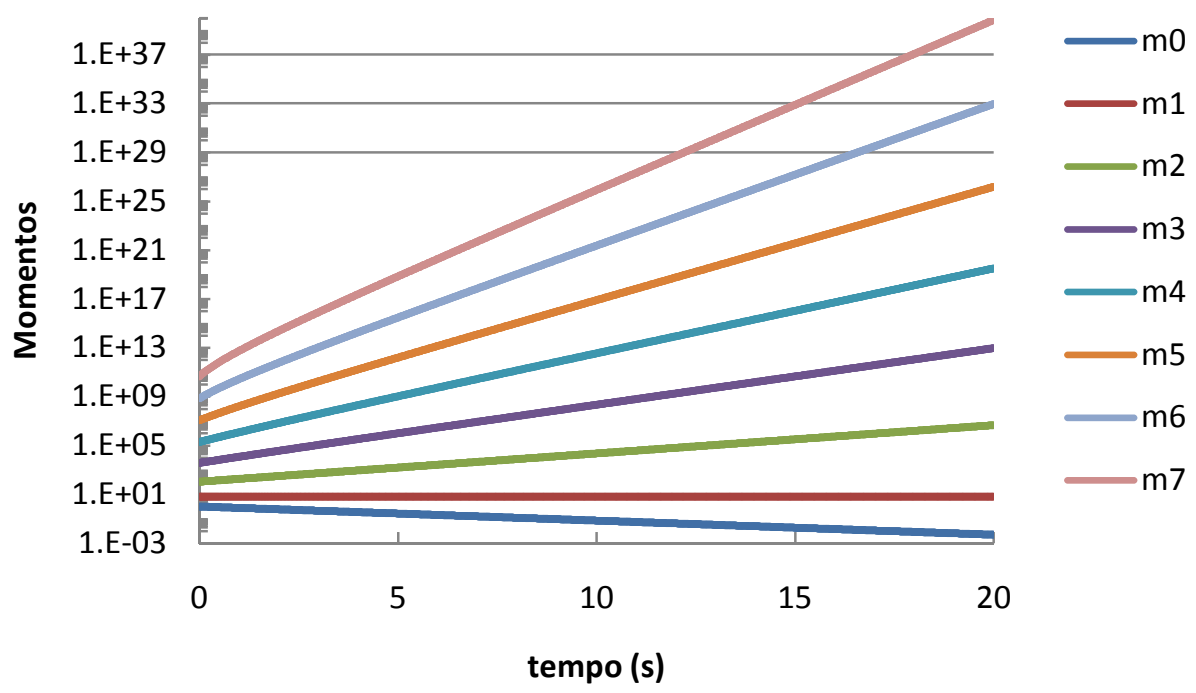

Figura 4.1.4 - Solução do QMOM proposto para um sistema sem escoamento ou difusão com agregação pura e Kernel Soma com $a=0,04$

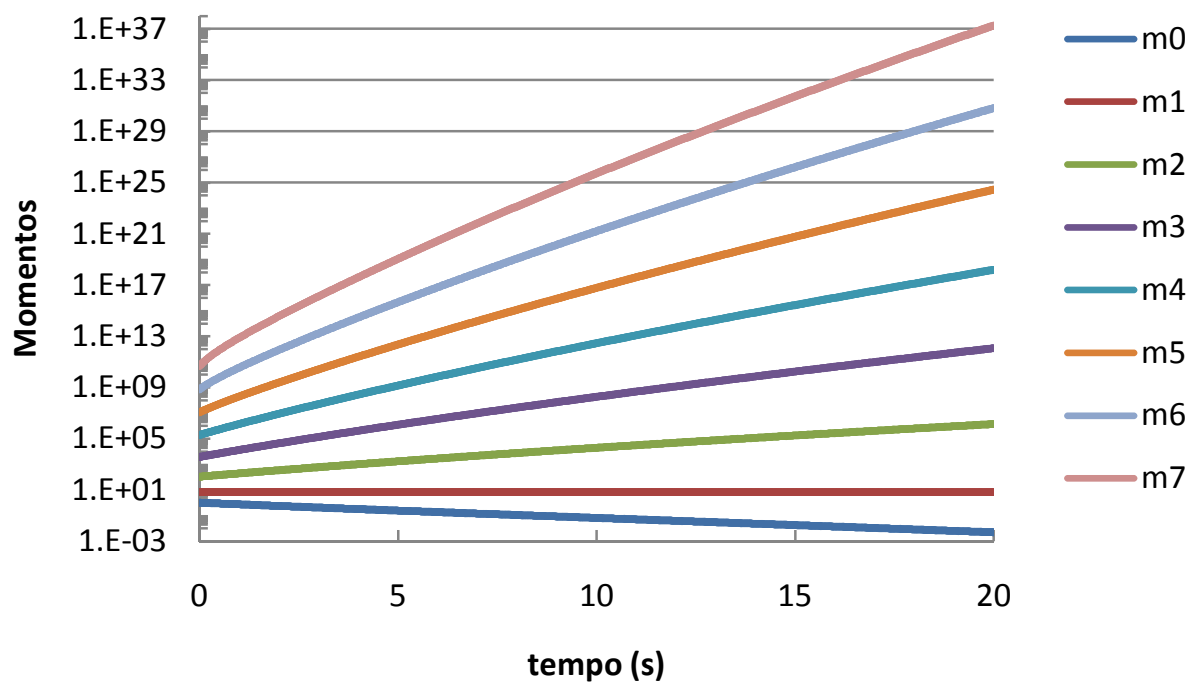

Figura 4.1.5 - Solução do QMOM proposto para um sistema sem escoamento ou difusão com agregação pura e Kernel Cisalhamento de Smoluchowsky com $a=$ 0,013 


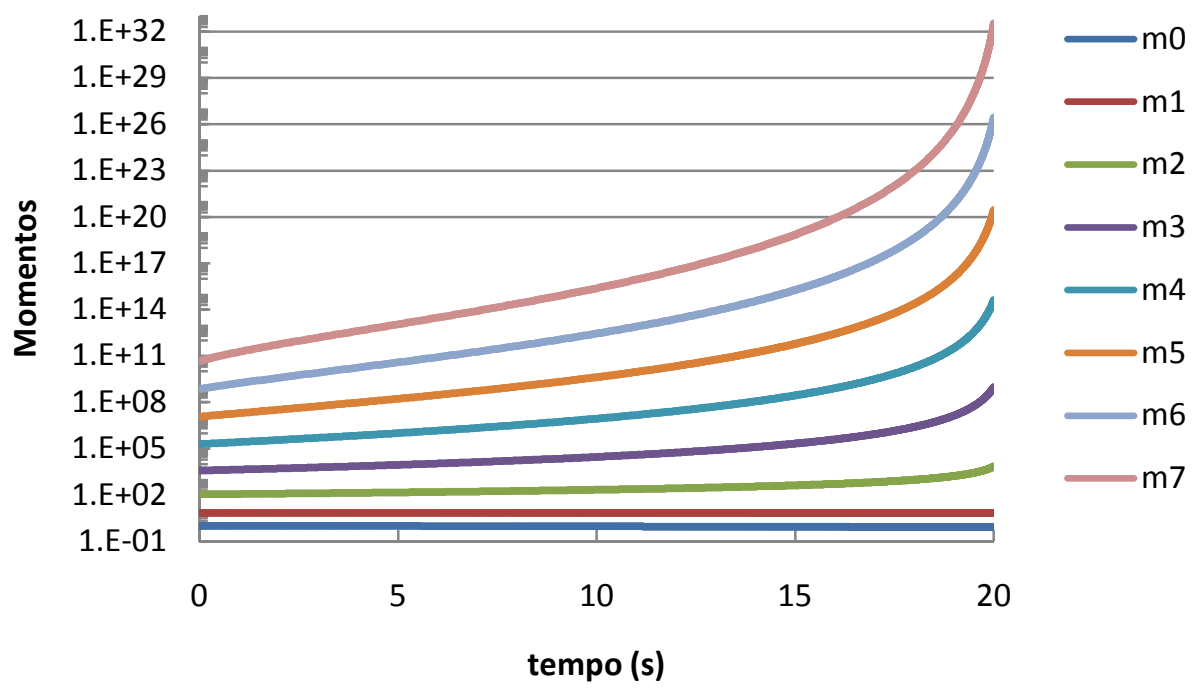

Figura 4.1.6 - Solução do QMOM proposto para um sistema sem escoamento ou difusão com agregação pura e Kernel Produto com $a=4,6 \cdot 10^{-4}$ e $b=1$

O kernel de Kapur produz um comportamento similar ao Constante, mas mais intenso. Um aumento de $b$ em relação a $c$ promove uma resposta mais suave, e vice-versa (Figuras 4.1.7 a 4.1.9).

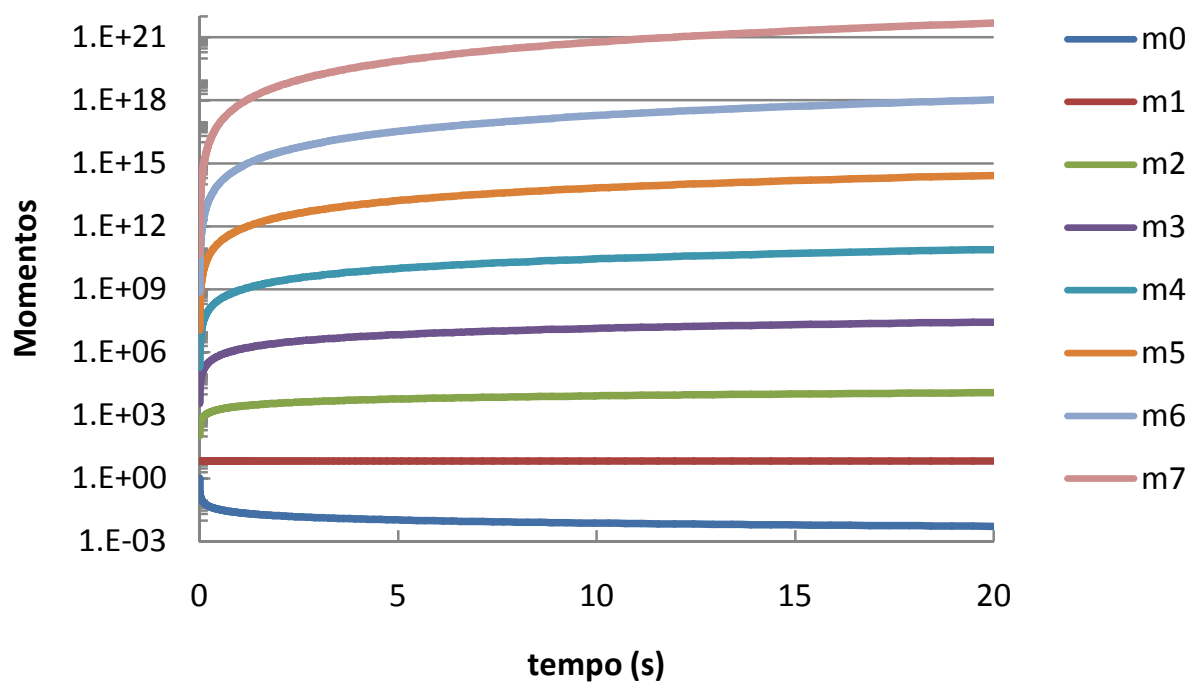

Figura 4.1.7 - Solução do QMOM proposto para um sistema sem escoamento ou difusão com agregação pura e Kernel de Kapur com $a=4300$ e $b=c=1$ 


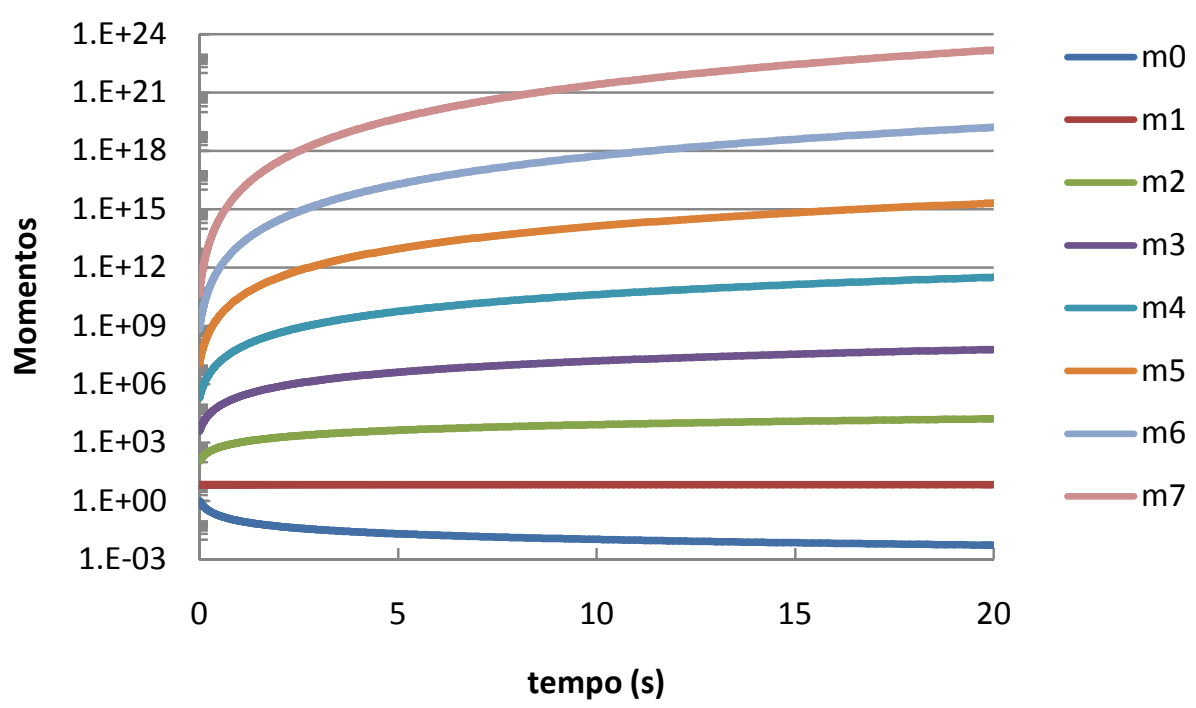

Figura 4.1.8 - Solução do QMOM proposto para um sistema sem escoamento ou difusão com agregação pura e Kernel de Kapur com $a=3,1, b=2$ e $c=1$

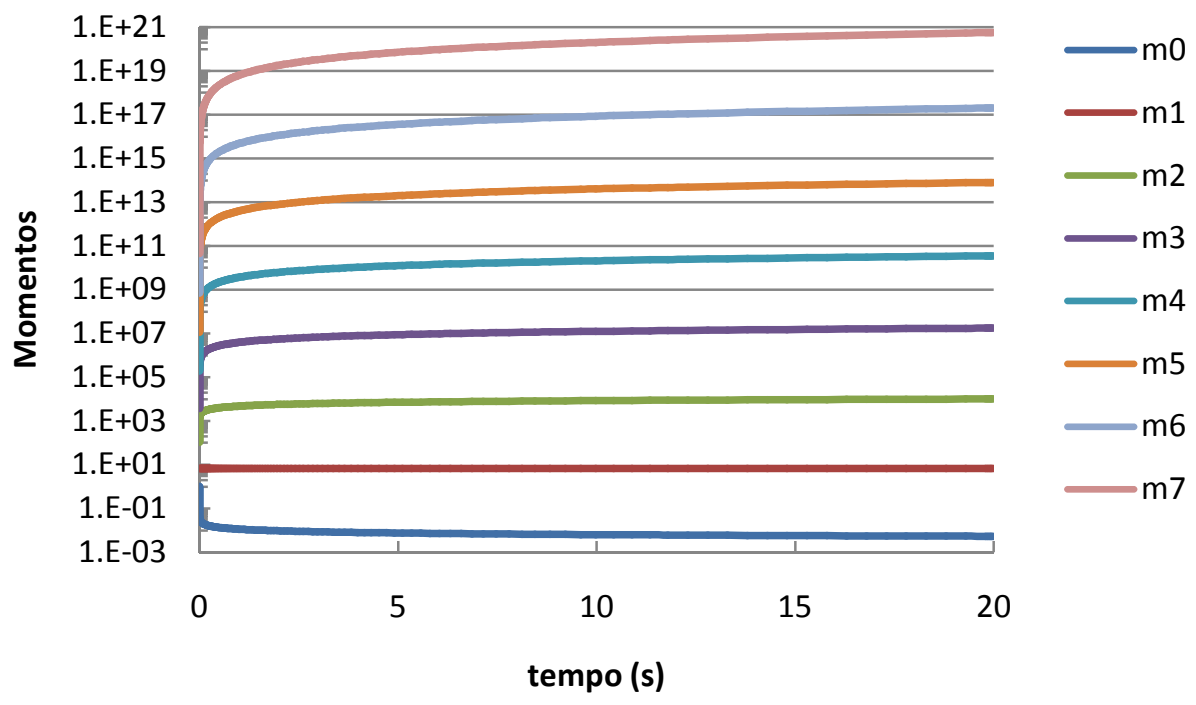

Figura 4.1.9 - Solução do QMOM proposto para um sistema sem escoamento ou difusão com agregação pura e Kernel de Kapur com $a=2,4.10^{9}, b=1$ e $c=2$

Finalmente, os kernéis de Sastry e de Adetayo e Ennis se comportam de maneira similar ao Soma (Figuras 4.1.10 e 4.1.11), com a diferença de que um aumento de $c$ em relação a $b$ no segundo faz com que sua resposta se aproxime àquela do Constante (Figura 4.1.12). 


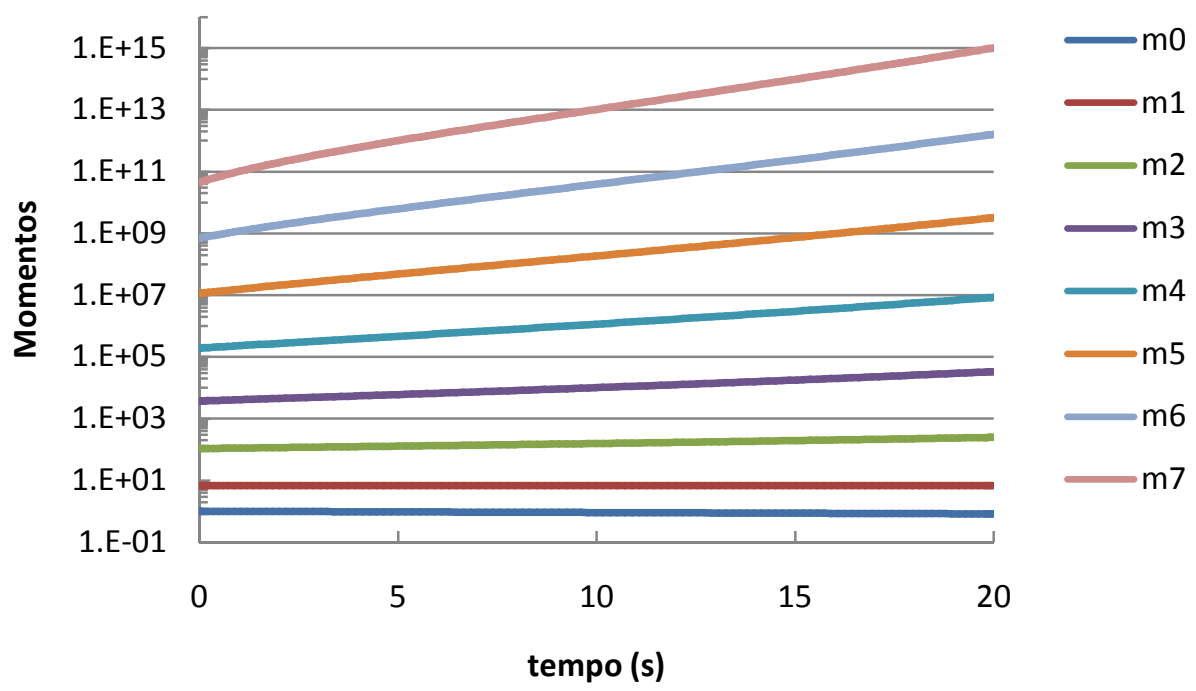

Figura 4.1.10 - Solução do QMOM proposto para um sistema sem escoamento ou difusão com agregação pura e Kernel de Sastry com $a=10^{-3}$

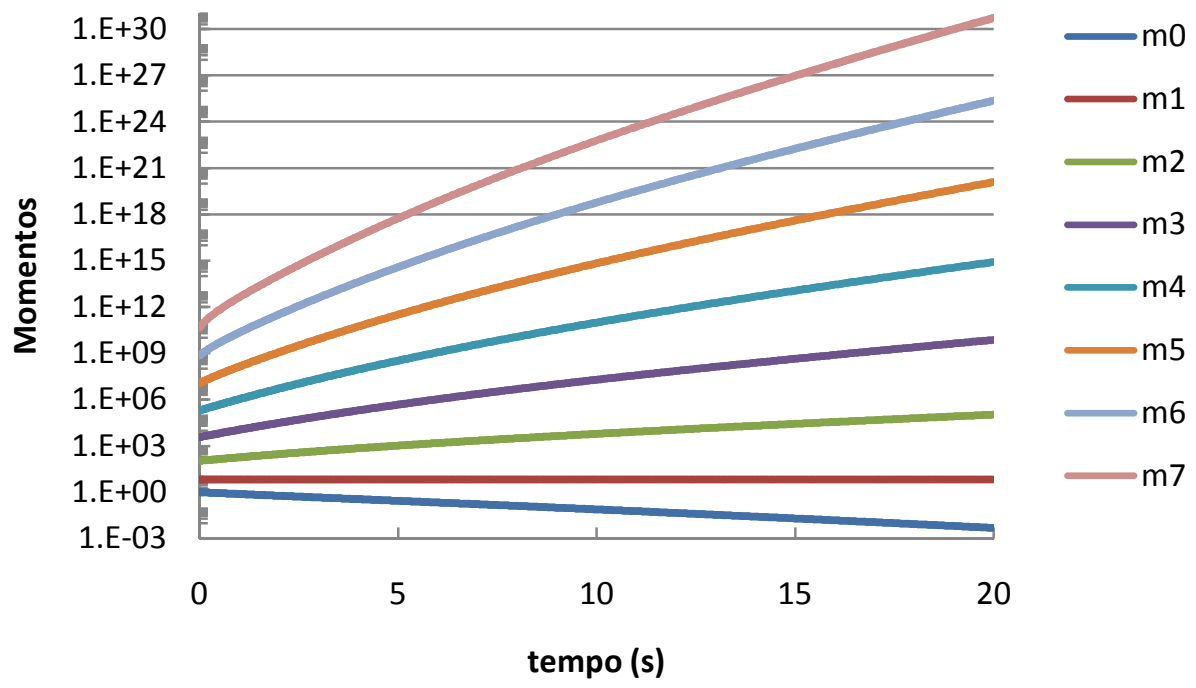

Figura 4.1.11 - Solução do QMOM proposto para um sistema sem escoamento ou difusão com agregação pura e Kernel de Adetayo e Ennis com $a=0,24, b=1 \mathrm{e}$ $c=1$ 


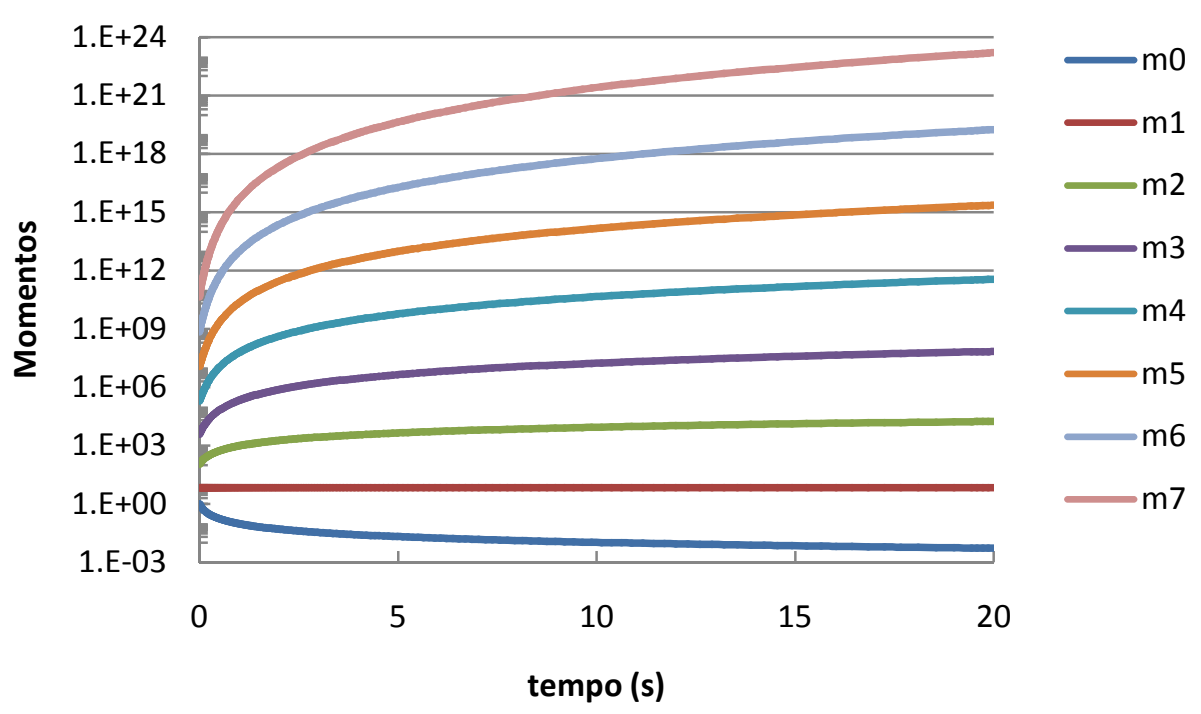

Figura 4.1.12 - Solução do QMOM proposto para um sistema sem escoamento ou difusão com agregação pura e Kernel de Adetayo e Ennis com $a=9,9 b=1$ e $c=2$

Dentre as possibilidades testadas, o kernel de Kapur com os parâmetros com $a=2,4 \cdot 10^{9}, b=1$ e $c=2$ foi o que melhor aproximou o comportamento previsto por Bresciani (2009). A formulação final do termo fonte do QMOM (e do DQMOM) é, então:

$$
\begin{gathered}
\bar{S}_{k}^{(N)}(t)=\bar{B}_{k}^{A}(t)-\bar{D}_{k}^{A}(t) \\
\bar{B}_{k}^{A}(\boldsymbol{Y}, t)=1,2.10^{9} \sum_{\alpha=1}^{N} \sum_{\beta=1}^{N} \omega_{\beta}(\boldsymbol{r}, t) \omega_{\alpha}(\boldsymbol{r}, t) \frac{\left[x_{\beta}(\boldsymbol{r}, t)+x_{\alpha}(\boldsymbol{r}, t)\right]^{k+1}}{\left[x_{\alpha}(\boldsymbol{r}, t) x_{\beta}(\boldsymbol{r}, t)\right]^{2}} \\
\bar{D}_{k}^{A}(\boldsymbol{Y}, t)=2,4.10^{9} \sum_{\alpha=1}^{N} \sum_{\beta=1}^{N} \omega_{\alpha}(\boldsymbol{r}, t) \omega_{\beta}(\boldsymbol{r}, t) \frac{x_{\alpha}{ }^{k-2}(\boldsymbol{r}, t)}{x_{\beta}{ }^{2}(\boldsymbol{r}, t)}\left[x_{\alpha}(\boldsymbol{r}, t)\right. \\
\left.+x_{\beta}(\boldsymbol{r}, t)\right]
\end{gathered}
$$

E os resultados obtidos com esse modelo são os mostrados na Tabela 4.1.3. 
Tabela 4.1.3 - Momentos finais obtidos pelo modelo proposto e seu erro relativo ao algoritmo de Bresciani (2009) para células de $400 \times 400, T=120^{\circ} \mathrm{C}, 7,0 \%$ de água, $E=1,0 \mathrm{kV} / \mathrm{cm}$, emulsão média e grandezas adimensionalizadas

\begin{tabular}{ccccc}
\hline$k$ & $m_{k}^{t=0,4 s}$ & Erro relativo (\%) & $m_{k}^{t=20 s}$ & Erro relativo (\%) \\
\hline 0 & $1,4038 \cdot 10^{-2}$ & 34,80 & $5,2769 \cdot 10^{-2}$ & 0,85 \\
1 & $6,6602 \cdot 10^{0}$ & 0,00 & $6,6602 \cdot 10^{0}$ & 0,00 \\
2 & $3,7347 \cdot 10^{3}$ & 99,66 & $9,9348 \cdot 10^{3}$ & 99,10 \\
3 & $2,4425 \cdot 10^{7}$ & 99,99 & $1,7283 \cdot 10^{8}$ & 99,99 \\
4 & $1,8364 \cdot 10^{10}$ & 100 & $3,4568 \cdot 10^{11}$ & 100 \\
5 & $1,5645 \cdot 10^{12}$ & 100 & $7,8341 \cdot 10^{13}$ & 100 \\
6 & $1,4916 \cdot 10^{15}$ & 100 & $1,9868 \cdot 10^{17}$ & 100 \\
7 & $1,5745 \cdot 10^{18}$ & 100 & $5,5789 \cdot 10^{20}$ & 100 \\
\hline
\end{tabular}

O modelo ajustado reproduz satisfatoriamente a evolução de $m_{0}$, que representa a distribuição de quantidade de gotas no sistema, e $m_{1}$, referente ao volume total da fase aquosa dispersa. Os momentos de ordem maior tendem a erros crescentes devido à natureza da aproximação por quadratura, mas são insignificantes para o entendimento do comportamento do sistema por serem desprovidos de significado físico relevante.

Como já discutido, a dinâmica do modelo de Bresciani (2009) não pôde ser validada. Pelo seu modelo, $t=0,4 \mathrm{~s}$ de exposição ao campo elétrico são suficientes para que quase toda a coalescência entre as gotas de água ocorra. Os $t=20 \mathrm{~s}$ necessários para que um volume de emulsão percorra toda a região dos eletrodos foram originados por um cálculo aproximado do escoamento, que pode ser facilmente verificado com técnicas de CFD. Descobrir se o tempo de residência da emulsão é maior que $t=0,4 \mathrm{~s}$ é, logo, necessário para comprovar que o modelo proposto pode aproximar o processo real. 


\subsection{Modelagem do Escoamento da Fase Contínua}

Relembrando o equacionamento dos balanços dos momentos:

$$
\frac{\partial m_{k}(\boldsymbol{r}, t)}{\partial t}+\nabla_{\mathbf{r}} \cdot\left[\dot{\boldsymbol{R}}(\boldsymbol{r}, \boldsymbol{Y}, t) m_{k}(\boldsymbol{r}, t)\right]=-\nabla \cdot \boldsymbol{j}_{k}(\boldsymbol{r}, \boldsymbol{Y}, t)+S_{k}(\boldsymbol{r}, \boldsymbol{Y}, t)
$$

Devido à existência da força elétrica entre as partículas, a contribuição à evolução da distribuição de partículas por fenômenos aleatórios é considerada desprezível. Logo, o termo difusivo, $\boldsymbol{j}_{k}(\boldsymbol{r}, \boldsymbol{Y}, t)$, como discutido na apresentação do equacionamento da EBP, é desconsiderado. O termo fonte $S_{k}(r, Y, t)$, por sua vez, foi equacionado na seção anterior. No entanto, ele só é válido para a emulsão escoando entre os eletrodos da dessalgadora. O conhecimento desse domínio de validade requer, então, a determinação do perfil de escoamento do sistema. Mais especificamente, do campo de velocidades da fase dispersa, $\dot{\boldsymbol{R}}(\boldsymbol{r}, \boldsymbol{Y}, t)$.

Bresciani (2009) contornou esse problema sugerindo uma aproximação para o perfil de escoamento no módulo de dessalgação, entre a válvula distribuidora e os últimos eletrodos do módulo (figura 4.2.1). Dada geometria do escoamento e a vazão de operação de $\dot{v}=2400 \mathrm{~m}^{3} /$ dia de emulsão, foram calculados a velocidade de injeção na saída da válvula, $v=1,18 \mathrm{~m} / \mathrm{s}$, e o tempo de residência da emulsão no volume de controle, $t_{r}=20 \mathrm{~s}$, para o qual os resultados do algoritmo de autômatos celulares já discutido foram validados.

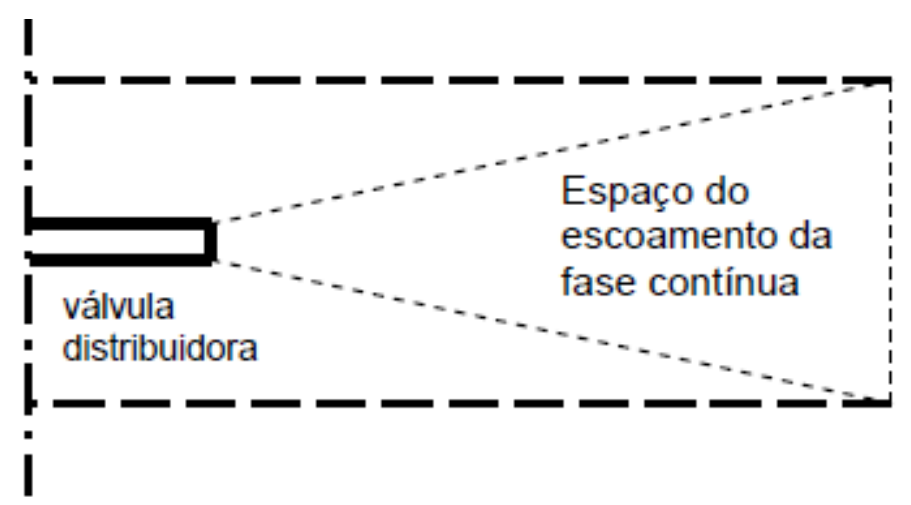

Figura 4.2.1 - Perfil de escoamento proposto por Bresciani (2009) 
O conhecimento desse perfil é fundamental para a validação dos dados apresentados, e a técnicas de CFD discutidas possibilitam uma aproximação mais segura do fenômeno real que a existente.

Para o início do estudo, algumas simplificações se fizeram necessárias. Primeiramente, foi considerado que a fase dispersa compartilha o campo de velocidades da fase contínua, e que a existência desta não afeta o escoamento da primeira. Ou seja, as forças de interação entre as fases são desprezíveis para a fase óleo (contínua), mas predominantes para a fase água (dispersa). A geometria complexa do módulo de dessalgação também precisou ser simplificada. A figura 4.2.2 mostra as medidas relatadas por Bresciani (2009).

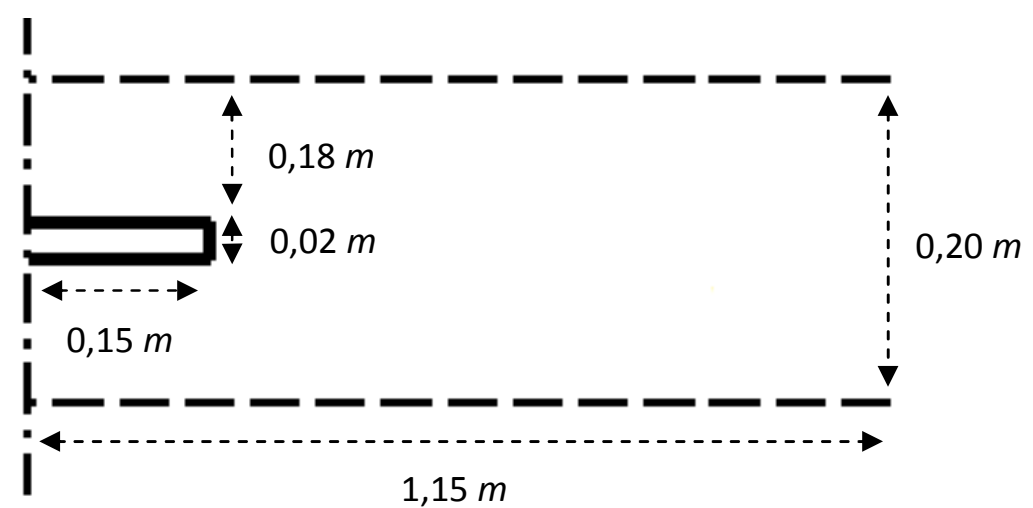

Figura 4.2.2 - Geometria do módulo de dessalgação apresentada por Bresciani (2009)

Pelas aproximações apresentadas, a simulação de um sistema monofásico é suficiente para se obter o perfil do escoamento. O fluido escolhido foi um petróleo líquido teórico com massa específica $\rho=792 \mathrm{~kg} / \mathrm{m}^{3}$, o mesmo do trabalho no qual esse estudo se baseou.

Foram estudadas duas geometrias principais: a apresentada na figura 4.2.3, que aproxima os eletrodos por uma parede plana, e a da figura 4.2.4, na qual os eletrodos são representados por feixes de seção quadrada. Em ambos os casos foram adotadas malhas bidimensionais com elementos quadrangulares, minimizando erros numéricos causados por elementos com vértices de ângulos muito distintos, comuns em malhas não-estruturadas. A seleção do tamanho dos elementos das malhas finais foi realizada de forma a minimizar os erros inerentes à discretização espacial, por tentativa e erro. 


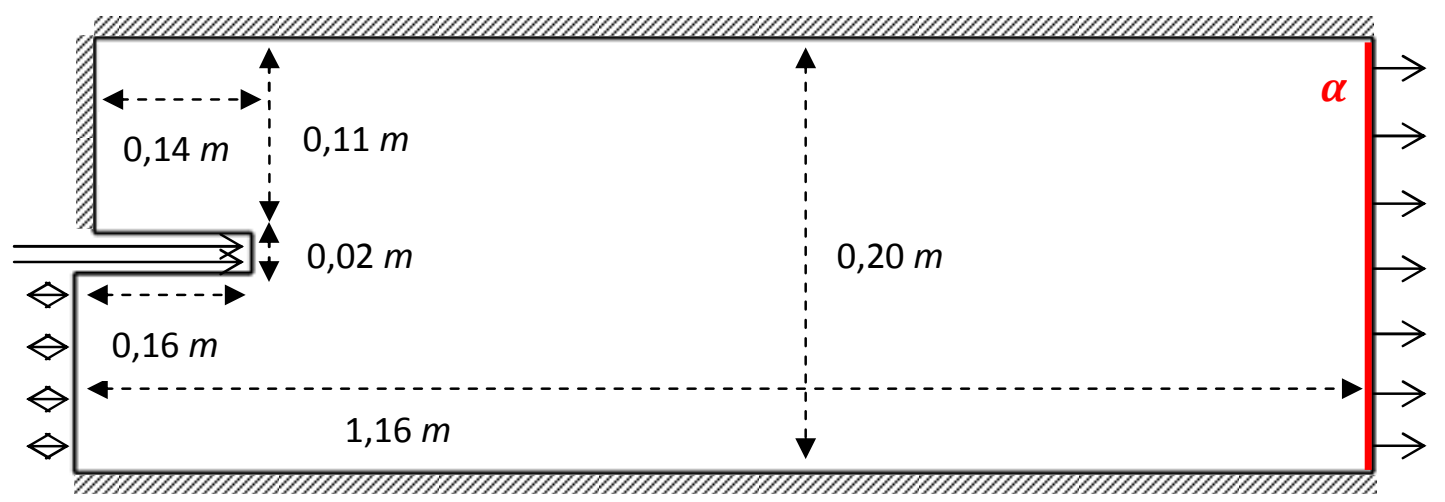

Figura 4.2.3 - Geometria da simulação de escoamento em CFD (A)

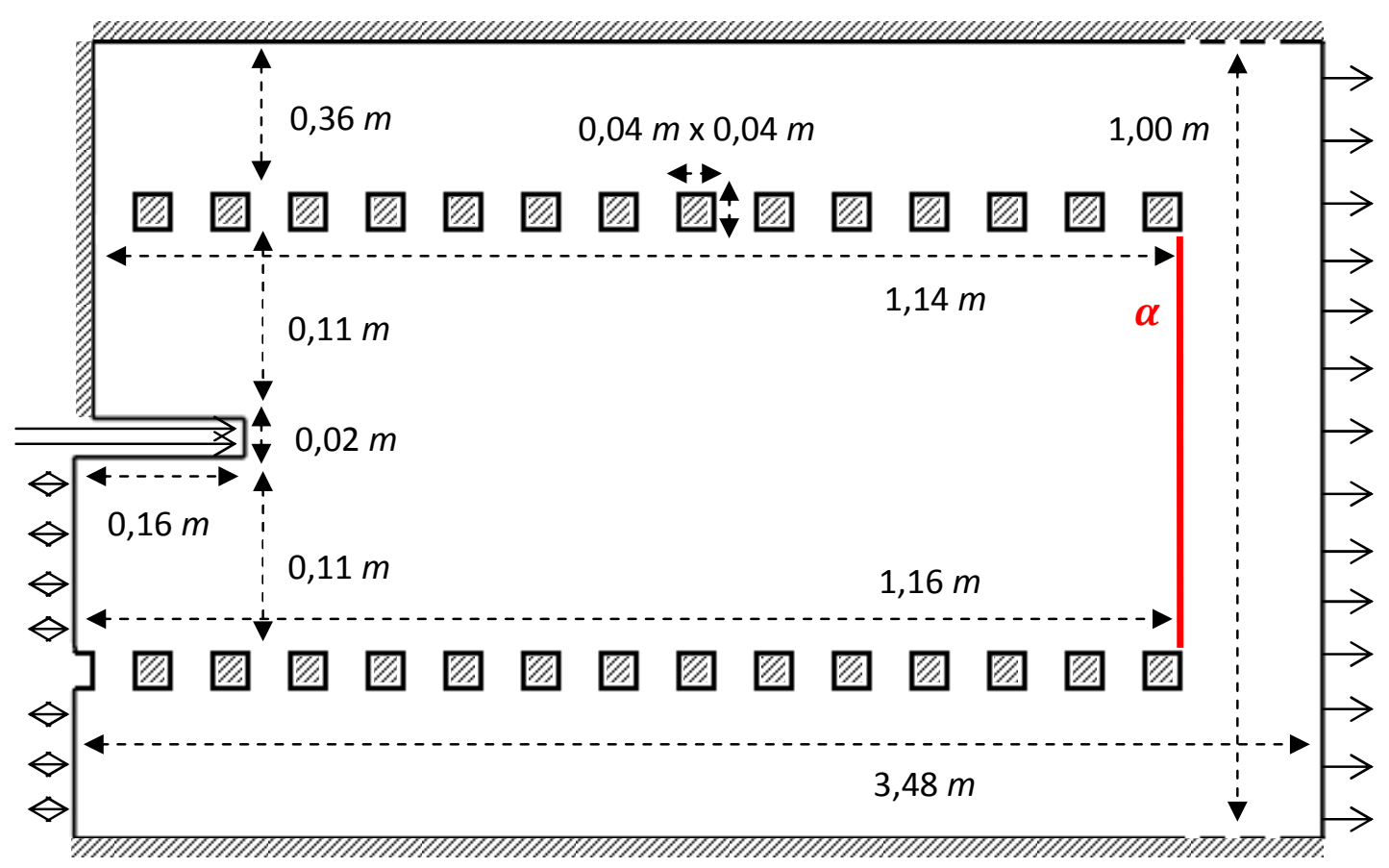

Figura 4.2.4 - Geometria da simulação de escoamento em CFD (B)

Os campos de velocidade e os tempos de residência do fluido do bocal do dispersor até a reta $\alpha$, para ambas as geometrias dadas, são mostrados nas figuras 4.2.5 a 4.2.10. Os tempos de residência foram obtidos plotando-se o tempo de escoamento sobre as linhas de fluxo das geometrias e extraindo os valores alcançados por essa grandeza na intersecção das linhas com a reta $\alpha$. 

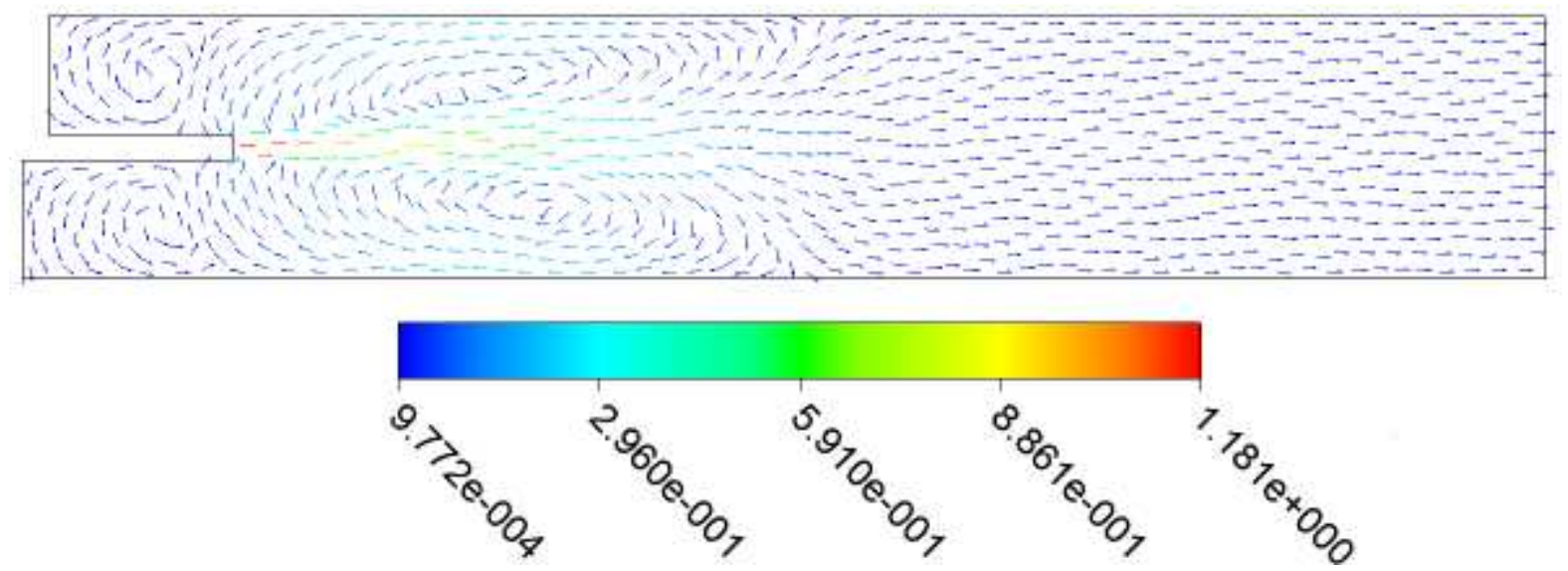

Figura 4.2.5 - Campo de velocidades calculado para a geometria (A)
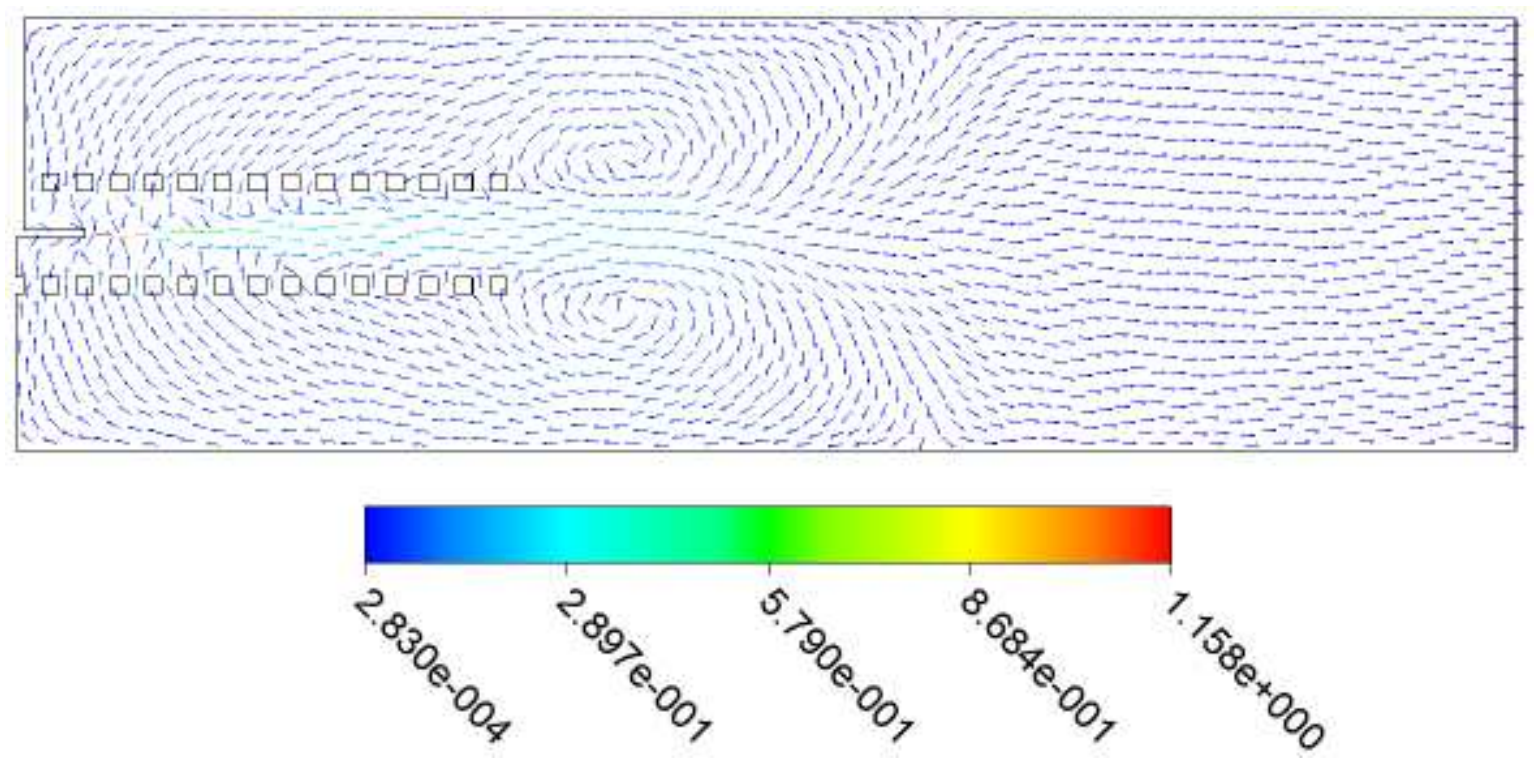

Figura 4.2.6 - Campo de velocidades calculado para a geometria (B) 

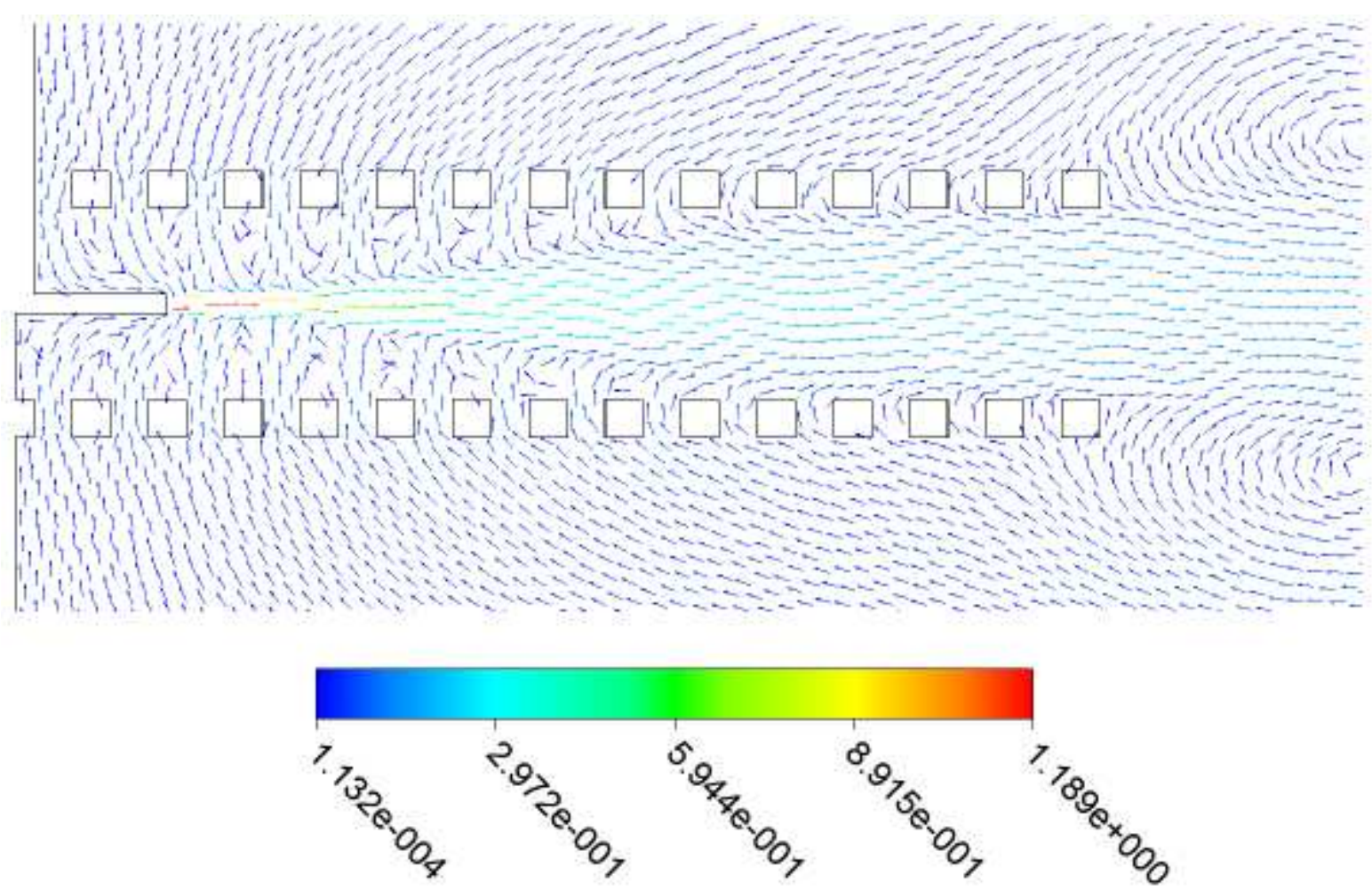

Figura 4.2.7 - Detalhe do campo de velocidades calculado para a geometria (B)

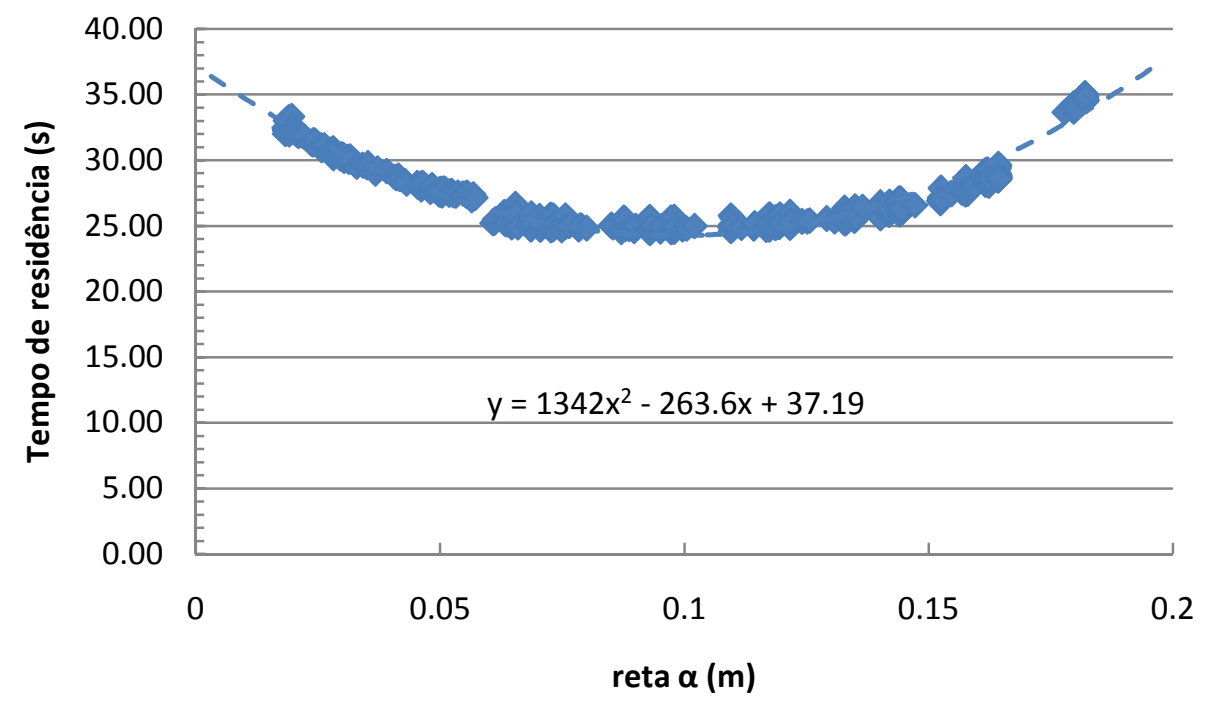

Figura 4.2.8 - Tempo de residência do fluido escoando da válvula distribuidora até a reta $\alpha$ para a geometria $(\mathrm{A})$ 


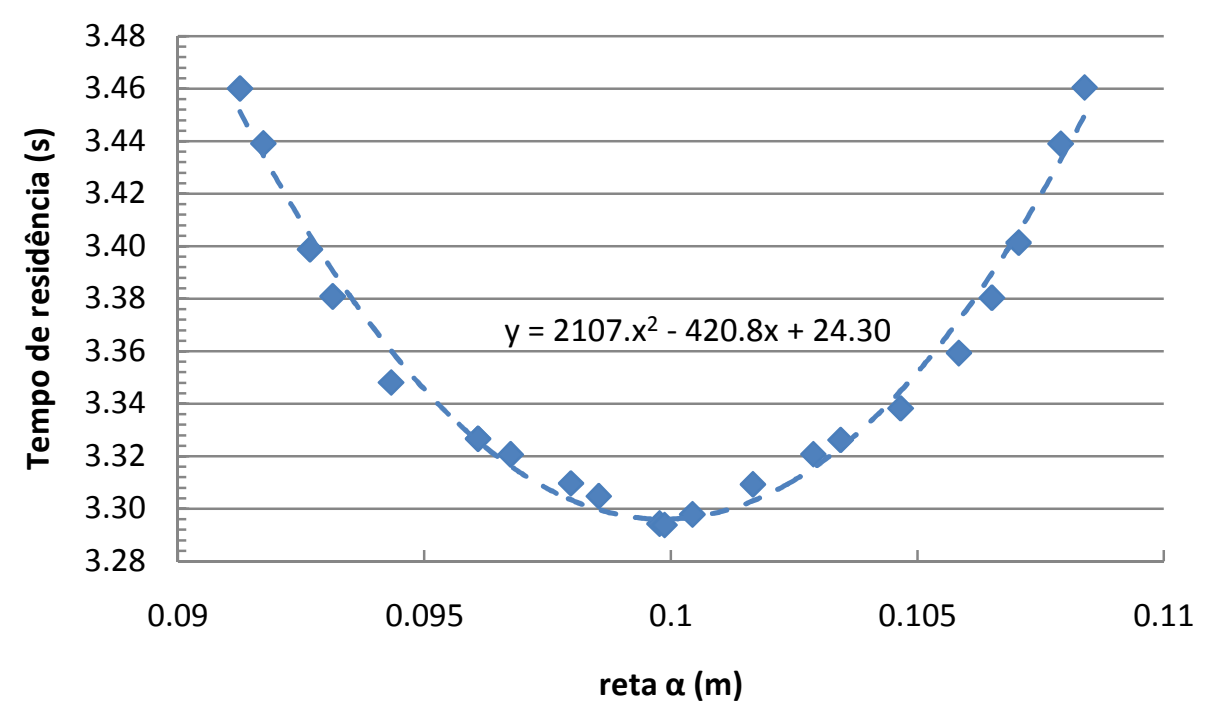

Figura 4.2.9 - Tempo de residência do fluido escoando da válvula distribuidora até a reta $\alpha$ para a geometria (B)

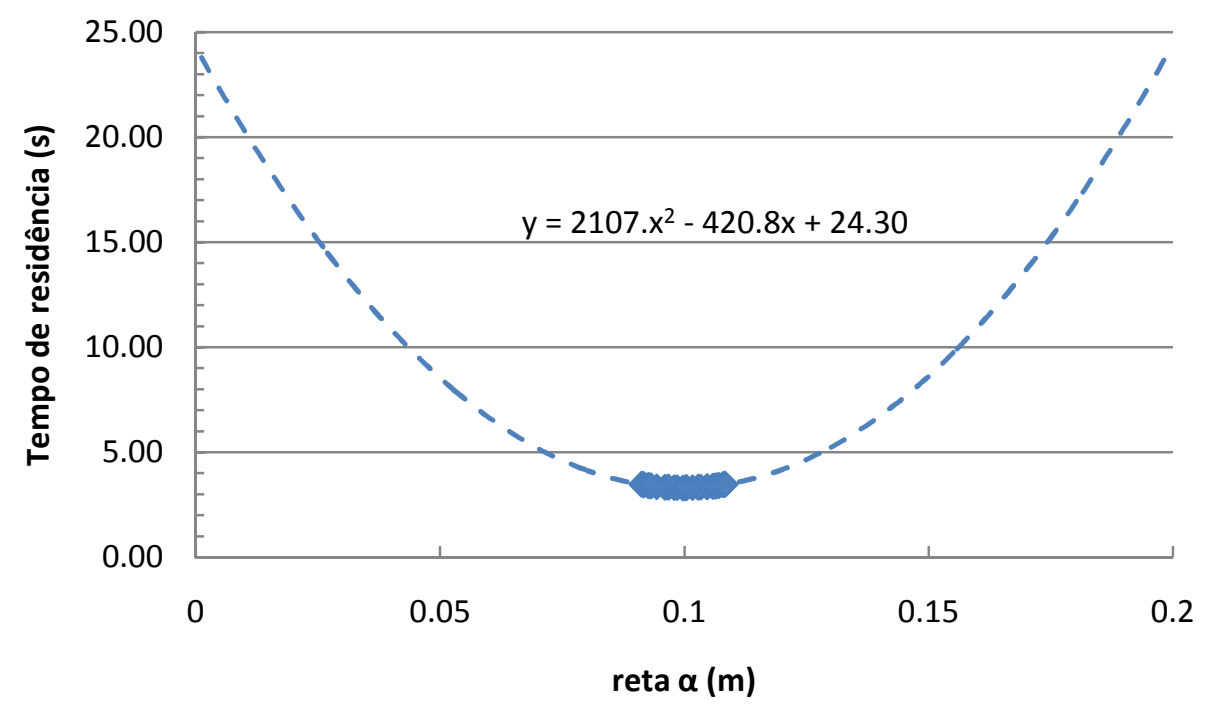

Figura 4.2.10 - Tempo de residência do fluido escoando da válvula distribuidora até a reta $\alpha$ para a geometria (B). Valores extrapolados por todos os $0,2 \mathrm{~m}$ do segmento de reta

A geometria (A) (figura 4.2.3) é uma reprodução da geometria simplificada proposta por Bresciani (2009). A simulação CFD corrobora, para esse caso, suas previsões . O campo de velocidades simulado (figura 4.2.5), apesar de diferente do sugerido (figura 4.2.1), produz um tempo de residência mínimo do fluido de 
aproximadamente $t=24 \mathrm{~s}$ (figura 4.2.8), próximo dos $t=20 \mathrm{~s}$ inicialmente considerados.

A geometria (B) (figura 4.2.4), por sua vez, busca uma aproximação mais realista do escoamento real, ao substituir as paredes representativas dos eletrodos por feixes que permitem a passagem de fluido. Apesar do perfil de escoamento figuras 4.2.6 e 4.2.7 ser mais condizente com o da previsão original (figura 4.2.1), o tempo de residência mínimo do fluido cai drasticamente para, aproximadamente, $t=3,3 \mathrm{~s}$. Isso pode ser explicado pela influência do fluido que recircula por entre os feixes de eletrodos, promovendo um desenvolvimento mais rápido do escoamento. Comparando os campos de velocidade de ambas as geometrias a diferente localização das zonas de recirculação é evidente.

As discrepâncias observadas sugerem um estudo mais preciso da geometria da dessalgadora, mas mesmo o caso mais conservador estudado (geometria (B)) produziu uma distribuição de tempos de residência maiores do que o necessário para invalidar o modelo de agregação, de $t=0,4 \mathrm{~s}$, como mostra a figura 4.2.11.

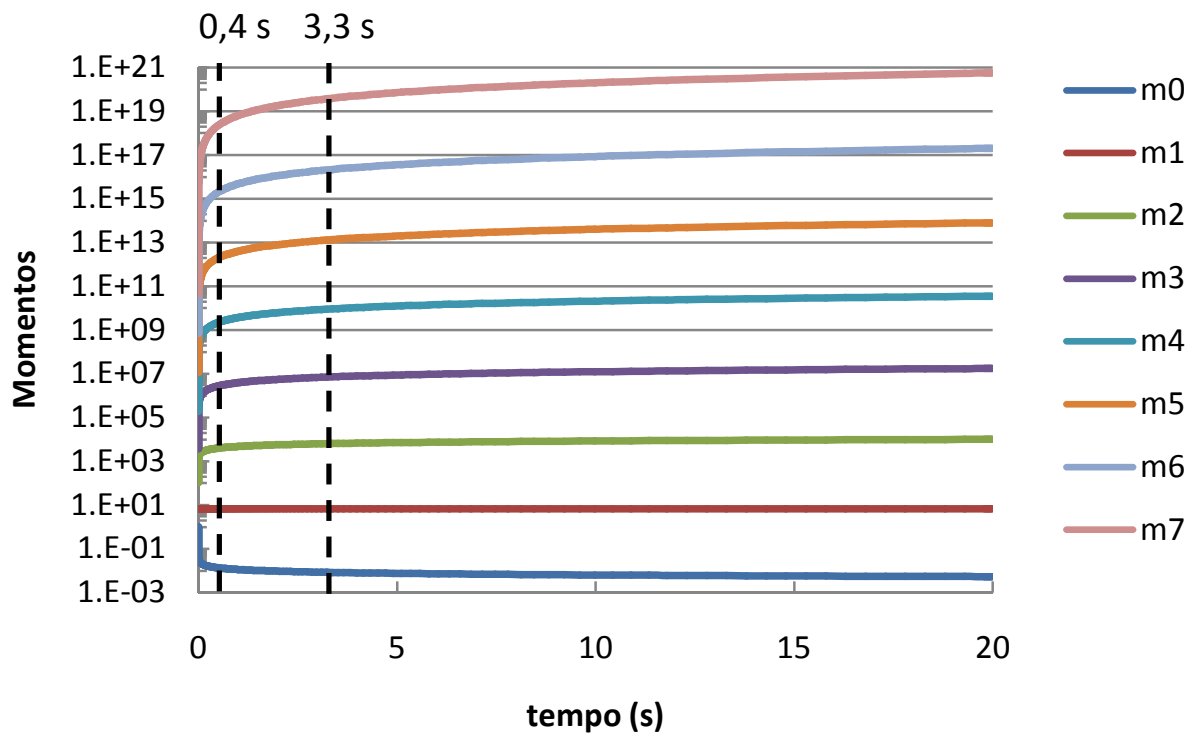

Figura 4.2.11 - Solução do modelo de agregação proposto, com indicação do tempo de residência mínimo requerido para utilização do modelo $(t=0,4 \mathrm{~s})$ e do obtido com a simulação das geometria $(\mathrm{A})(t=3,3 \mathrm{~s})$ 


\section{CONCLUSÕES}

O equacionamento de transporte genérico de sistemas bifásicos polidispersos foi discutido e simplificado de acordo com práticas comuns na literatura, sendo este composto por equações de transporte de cada fase e uma equação de interface, a EBP. Os principais métodos dos momentos, utilizados para a solução da equação do balanço populacional, foram equacionados e suas vantagens e desvantagens listadas.

Os algoritmos dos métodos propostos para a solução da EBP (QMOM e DQMOM) foram implementados em Matlab e linguagem C. Equivalência dos resultados obtidos à solução analítica proposta por McCoy e Madras (2003) validaram sua aplicação.

Importantes informações sobre o comportamento numérico do DQMOM foram obtidas, como o fato de que os erros numéricos para processos de coalescência (nos moldes do modelo de validação estudado) não se estabilizam com o tempo de simulação. A sensibilidade do sistema em relação à forma da função freqüência de agregação foi mostrada significativa pelo estudo de diferentes kerneis propostos na literatura. A complexidade do fenômeno de agregação no interior de uma dessalgadora impediu que um kernel físico específico para esse fenômeno pudesse, no escopo deste, ser gerado. Para tanto é necessário um conhecimento da distribuição de distâncias entre partículas por toda a emulsão.

Considerando, para fins de estudo, o algoritmo de Bresciani (2009) como uma reprodução fiel do comportamento de uma emulsão água-óleo no interior de uma dessalgadora, foi gerado um modelo que traduz satisfatoriamente o fenômeno para o equacionamento de balanço populacional. Esse resultado pode, futuramente, ser utilizado em conjunto das equações de transporte bifásicas aqui apresentadas para um estudo mais completo do escoamento no processo aqui exemplificado.

No intuito de ampliar a confiança no algoritmo de autômatos celulares apresentado por Bresciani (2009), foi realizado um estudo simplificado do escoamento no interior de um módulo de dessalgação auxiliado por técnicas de CFD. De acordo com os resultados obtidos, o tempo de residência do fluido é suficiente para que, de acordo com a dinâmica do modelo proposto, ocorra agregação entre as gotas d'água suficiente para coerência com o processo real. 
Finalmente, o escopo proposto de apresentação do equacionamento da coalescência em sistemas bifásicos polidispersos genéricos e seu exemplo de aplicação para o caso da dessalgação de petróleo foi atendido e possibilidades de estudos mais aprofundados tanto sobre o modelo de agregação quanto sobre a simulação do escoamento da emulsão foram apresentadas. 


\section{REFERÊNCIAS}

ADETAYO, A. A., ENNIS, B. J. Unifying Approach to Modeling Granule Coalescence Mechanisms. A.I.Ch.E Journal, v. 43, p. 927-934, 1997.

ATTARAKIH, M. M., BART, H., FAQIR, N. M. Numerical solution of the bivariate population balance equation for the interacting hydrodynamics and mass transfer in liquid-liquid extraction columns. Chemical Engineering Science, v. 61, p. 113-123, 2006.

ATTARAKIH, M. M., DRUMM, C., BART, H. Solution of the population balance equation using the sectional quadrature method of moments (SQMOM). Chemical Engineering Science, v. 64, p. 742-752, 2009.

BARRET, J. C.; WEBB, N. A. A comparison of some approximate methods for solving the aerosol general dynamic equation. Journal of Aerosol Science, v. 29, p. 31-39, 1998.

BIRD, R. B., STEWART, W. E., LIGHTFOOT, E. N. Transport Phenomena: Second Edition. New York, NY, USA: John Wiley \& Sons, Inc., 2002.

BENNETT, C. O., MYERS, J. E. Momentum, Heat and Mass Transfer. Columbus, OH, USA: McGraw-Hill, Inc., 1962.

BHARDWAJ, A., HARTLAND, S. Dynamics of emulsification and demulsification of water in crude oil emulsions. Industrial \& Engineering Chemistry Research, v. 33, p. 1271-1279, 1994.

BOVE, S., SOlBERG, T., HJERTAGER, B. H. A novel algorithm for solving population balance equations: the parallel parent and daughter classes. Derivation, analysis and testing. Chemical Engineering Science, v. 60, p. 14491464, 2005.

BRESCIANI, A. E. Análise do processo de dessalgação de petróleo. Otimização do uso de água. Tese (Doutorado) - Escola Politécnica, Universidade de São Paulo, São Paulo, SP, Brasil, 2009. 
BUTKOV, E. Mathematical Physics. Reading, MA, USA: Addison-Wesley Publishing Company, Inc., 1968.

CAMPOS, F. B., LAGE, P. L. C. A numerical method for solving the transient multidimensional population balance equation using na Euler-Lagrange formulation. Chemical Engineering Science, v. 58, p. 2725-2744, 2003.

DAMIAN, R. B. Acoplamento de balanço populacional à simulação computacional de escoamentos multifásicos polidispersos. Dissertação (Mestrado) - COPPE, Universidade Federal do Rio de Janeiro, Rio de Janeiro, RJ, Brasil, 2007.

DRUMM, C., ATTARAKIH, M. M., BART, H. J. Coupling of CFD with DPBM for an RDC extractor. Chemical Engineering Science, v. 64, p. 721-732, 2009.

FAN, R., MARCHISIO, D. L., FOX, R. O. Application of the direct quadrature method of moments to polydisperse gas-solid fluidized beds. Powder Technology, v. 139, p. 7-20, 2004.

FOX, R. O. Bivariate direct quadrature method of moments for coagulation and sintering of particle populations. Aerosol Science, v. 37, p. 1562-1580, 2006.

FOX, R. O., LAURENT, F., MASSOT, M. Numerical simulation of spray coalescence in an Eulerian framework: Direct quadrature method of moments and multi-fluid method. Journal of Computational Physics, v. 227, p. 3058-3088, 2008.

FRENKLACH, M., HARRIS, S. J. Aerosol Dynamics Modeling Using the Method of Moments. Journal of Colloid and Interface Science, v. 118, p. 252-261, 1987.

GIDASPOW, D. Multiphase Flow and Fluidization: Continuum and Kinetic Theory Descriptions. San Diego, CA, USA: Academic Press, Inc., 1994.

GORDON, R. G. Error Bounds in Equilibrium Statistical Mechanics. Journal of Mathematical Physics, v. 9, p. 655-672, 1968.

GU, Z. L., SU, J. W., JIAO, J. Y., XU, X. Y. Simulation of micro-behaviors including nucleation, growth, and aggregation in particle system. Science in China Series B: Chemistry, v. 52, p. 241-248, 2009. 
HOUNSLOW, M. J., NI, X. Population balance modelling of droplet coalescence and break-up in an oscillatory baffled reactor. Chemical Engineering Science, v. 59, p. 819-828, 2004.

HULBURT, H. M. \& KATZ, S. Some problems in particle technology: A statistical mechanical formulation. Chemical Engineering Science, v. 19, p. 555-574, 1964.

ISHII, M., HIBIKI, T. Thermo-fluid Dynamics of Two-phase Flow. New York, NY, USA: Springer Science+Business Media, LCC., 2006.

KAPUR, P. C. Kinetics of granulation by non-random coalescence mechanism. Chemical Engineering Science, v. 27, p. 1863-1869, 1972.

KUMAR, S., RAMKRISHNA, D. On the solution of population balance equations by discretization - I. A fixed pivot technique. Chemical Engineering Science, v. 51, p. 1311-1332, 1996.

KUMAR, J., PEGLOW, M., WARNECKE, G., HEINRICH, S., MÖRL, L. Improved accuracy and convergence of discretized population balance for aggregation: The cell average technique. Chemical Engineering Science, v. 61, p. 3327-3342, 2006.

LIU, L. X., LITSTER, J. D. Population balance modelling of granulation with a physically based coalescence kernel. Chemical Engineering Science, v. 57, p. 2183-2191, 2002.

MALISKA, C. R. Transferência de Calor e Mecânica dos Fluidos Computacional: Fundamentos e Coordenadas Generalizadas, Rio de Janeiro, RJ, Brasil: Livros Técnicos e Científicos Editora S. A., 1995.

MARCHISIO, D. L., VIRGIL, R. D., FOX, R. O. Quadrature method of moments for aggregation-breakage processes. Journal of Colloid \& Interface Science, v.258, p. 322-334, 2003a.

MARCHISIO, D. L., PITKURNA, J.T., FOX, R. O., VIRGIL, R. D. Quadrature Method of Moments for Population-Balance Equations. A.I.Ch.E Journal, v.49, p. 1266-1276, 2003b. 
MARCHISIO, D. L., VIRGIL, R. D., FOX, R. O. Implementation of the quadrature method of moments in CFD for aggregation-breakage problems. Chemical Engineering Science, v. 58, p. 3337-3351, 2003c.

MARCHISIO, D. L., FOX, R. O. Solution of population balance equations using the direct quadrature method of moments. Aerosol Science, v. 36, p. 43-73, 2005.

MCCOY, B. J., MADRAS, G. Analytical Solution for a population balance equation with aggregation and fragmentation. Chemical Engineering Science, $v$. 58, p. 3049-3051, 2003.

MCGRAW, R. Description of Aerosol Dynamics by the Quadrature Method of Moments. Aerosol Science and Technology, v. 27, p. 255-265, 1997.

PATANKAR, S. V. Numerical heat transfer and fluid flow. New York, NY, USA: Hemisphere Publishing Corporation, 1980.

RAMKRISHNA, D. Population Balances: Theory and Applications to Particulate Systems in Engineering. San Diego, CA, USA: Academic Press, Inc., 2000.

RAMKRISHNA, D., MAHONEY, A. W. Population Balance Modeling. Promise for the future. Chemical Engineering Science, v. 57, p. 595-606, 2002.

SILVA, L. F. L. R., DAMIAN, R. B., LAGE, P. L. C. Implementation and analysis of numerical solution of the population balance equation in CFD packages. Computers and Chemical Engineering, v. 32, p. 2933-2945, 2008.

SU, J., GU, Z., LI, Y., FENG, S., XU, X. Y. Solution of population balance equation using quadrature method of moments with an adjustable factor. Chemical Engineering Science, v. 62, p. 5897-5911, 2007.

SU, J., GU, Z., LI, Y., FENG, S., XU, X. Y. An Adaptive Direct Quadrature Method of Moment for Population Balance Equations. A.I.Ch.E Journal, v. 54, p. 28722887, 2008.

SU, J. W., GU, Z. L., XU, X. Y. Advances in numerical methods for the solution of population balance equations for disperse phase systems. Science in China Series B: Chemistry, v. 52, p. 1063-1079, 2009. 
VERSTEEG, H. K., MALALASEKERA, W. An Introduction to Computational Fluid Dynamics. Harlow, Essex, UK: Pearson Education Limited, 1995.

WAN, B., RING, T. A. Verification of SMOM and QMOM population balance modeling in CFD code using analytical solutions for batch particulate processes. China Particuology, v. 4, p. 243-249, 2006.

WRIGHT, D. L., MCGRAW, R., ROSNER, D. E. Bivariate Extension of the Quadrature Method of Moments for Modeling Simultaneous Coagulation and Sintering of Particle Populations. Journal of Colloid and Interface Science, v. 236, p. 242-251, 2001.

ZUCCA, A., MARCHISIO, D. L., BARRESI, A. A., FOX, R. O. Implementation of the population balance equation in CFD codes in modelling soot formation in turbulent flames. Chemical Engineering Science, v. 61, p. 87-95, 2006. 


\section{APÊNDICE A \\ DEMONSTRAÇÃO DA CONSERVAÇÃO DO MOMENTO DE PRIMEIRA ORDEM}

Dadas as equações 4.1.4 a 4.1.7, que descrevem os balanços de momentos para um caso sem escoamento e termo fonte composto somente pelo fenômeno de agregação de partículas:

$$
\frac{\partial m_{k}}{\partial t}=\frac{1}{2} \sum_{\alpha=1}^{N} \sum_{\beta=1}^{N} \omega_{\beta} \omega_{\alpha}\left[x_{\beta}+x_{\alpha}\right]^{k} a_{\beta \alpha}-\sum_{\alpha=1}^{N} \sum_{\beta=1}^{N} \omega_{\alpha} \omega_{\beta} x_{\alpha}{ }^{k} a_{\beta \alpha}
$$

O balanço do momento de primeira ordem $m_{1}$ é escrito:

$$
\frac{\partial m_{1}}{\partial t}=\frac{1}{2} \sum_{\alpha=1}^{N} \sum_{\beta=1}^{N} \omega_{\beta} \omega_{\alpha}\left[x_{\beta}+x_{\alpha}\right] a_{\beta \alpha}-\sum_{\alpha=1}^{N} \sum_{\beta=1}^{N} \omega_{\alpha} \omega_{\beta} x_{\alpha} a_{\beta \alpha}
$$

E pode ser simplificado para:

$$
\frac{\partial m_{1}}{\partial t}=\frac{1}{2} \sum_{\alpha=1}^{N} \sum_{\beta=1}^{N} \omega_{\beta} \omega_{\alpha} x_{\beta} a_{\beta \alpha}-\frac{1}{2} \sum_{\alpha=1}^{N} \sum_{\beta=1}^{N} \omega_{\alpha} \omega_{\beta} x_{\alpha} a_{\beta \alpha}
$$

Para $N=1$ pontos de quadratura:

$$
\frac{\partial m_{1}^{(1)}}{\partial t}=\frac{1}{2} \omega_{1} \omega_{1} x_{1} a_{11}-\frac{1}{2} \omega_{1} \omega_{1} x_{1} a_{11}=0
$$

Para $N=2$ pontos de quadratura: 


$$
\begin{gathered}
\frac{\partial m_{1}^{(2)}}{\partial t}=\frac{1}{2}\left[\omega_{1} \omega_{1} x_{1} a_{11}+\omega_{2} \omega_{1} x_{2} a_{21}+\omega_{1} \omega_{2} x_{1} a_{12}+\omega_{2} \omega_{2} x_{2} a_{22}\right] \\
-\frac{1}{2}\left[\omega_{1} \omega_{1} x_{1} a_{11}+\omega_{1} \omega_{2} x_{1} a_{21}+\omega_{2} \omega_{1} x_{2} a_{12}\right. \\
\left.+\omega_{2} \omega_{2} x_{2} a_{22}\right] \\
\Rightarrow \frac{\partial m_{1}^{(2)}}{\partial t}=\frac{1}{2}\left[\omega_{2} \omega_{1}\left[x_{2}-x_{1}\right] a_{21}+\omega_{1} \omega_{2}\left[x_{1}-x_{2}\right] a_{12}\right]
\end{gathered}
$$

Como $a_{21}=a_{12}$ (equação 2.3.1.1), segue:

$$
\frac{\partial m_{1}^{(2)}}{\partial t}=0
$$

Para $N=3$ pontos de quadratura:

$$
\begin{gathered}
\frac{\partial m_{1}^{(3)}}{\partial t}=\frac{1}{2}\left[\omega_{1} \omega_{1} x_{1} a_{11}+\omega_{2} \omega_{1} x_{2} a_{21}+\omega_{3} \omega_{1} x_{3} a_{31}+\omega_{1} \omega_{2} x_{1} a_{12}\right. \\
+\omega_{2} \omega_{2} x_{2} a_{22}+\omega_{3} \omega_{2} x_{3} a_{32}+\omega_{1} \omega_{3} x_{1} a_{13}+\omega_{2} \omega_{3} x_{2} a_{23} \\
\left.+\omega_{3} \omega_{3} x_{3} a_{33}\right] \\
-\frac{1}{2}\left[\omega_{1} \omega_{1} x_{1} a_{11}+\omega_{1} \omega_{2} x_{1} a_{21}+\omega_{1} \omega_{3} x_{1} a_{31}\right. \\
+\omega_{2} \omega_{1} x_{2} a_{12}+\omega_{2} \omega_{2} x_{2} a_{22}+\omega_{2} \omega_{3} x_{2} a_{32}+\omega_{3} \omega_{1} x_{3} a_{13} \\
\left.+\omega_{3} \omega_{2} x_{3} a_{23}+\omega_{3} \omega_{3} x_{3} a_{33}\right] \\
\Rightarrow \frac{\partial m_{1}^{(3)}}{\partial t}=\frac{1}{2}\left[\omega_{2} \omega_{1}\left[x_{2}-x_{1}\right] a_{21}+\omega_{3} \omega_{1}\left[x_{3}-x_{1}\right] a_{31}\right. \\
+\omega_{1} \omega_{2}\left[x_{1}-x_{2}\right] a_{12}+\omega_{3} \omega_{2}\left[x_{3}-x_{2}\right] a_{32} \\
\left.+\omega_{1} \omega_{3}\left[x_{1}-x_{3}\right] a_{13}+\omega_{2} \omega_{3}\left[x_{2}-x_{3}\right] a_{23}\right]
\end{gathered}
$$

Novamente, a função frequência de agregação é simétrica (equação 2.3.1.1). Ou seja, $a_{21}=a_{12}, a_{31}=a_{13}$ e $a_{32}=a_{23}$. Assim sendo:

$$
\frac{\partial m_{1}^{(3)}}{\partial t}=0
$$


A expansão dos somatórios permite a visualização de um padrão que se repete para qualquer número de pontos de quadratura adotado. Os termos que multiplicam kernéis de agregação simétricos entre si sempre se cancelam. Pode-se concluir, então, que o momento de primeira ordem, $m_{1}$, é sempre conservado, dado o equacionamento proposto. 